\title{
Laser-Plasma Interactions \\ Relevant to Inertial \\ Confinement Fusion
}

\author{
K. B. Wharton \\ (Ph.D. Dissertation)
}

November 1998 


\section{DISCLAIMER}

This document was prepared as an account of work sponsored by an agency of the United States Government. Neither the United States Government nor the University of California nor any of their employees, makes any warranty, express or implied, or assumes any legal liability or responsibility for the accuracy, completeness, or usefulness of any information, apparatus, product, or process disclosed, or represents that its use would not infringe privately owned rights. Reference herein to any specific commercial product, process, or service by trade name, trademark, manufacturer, or otherwise, does not necessarily constitute or imply its endorsement, recommendation, or favoring by the United States Government or the University of California. The views and opinions of authors expressed herein do not necessarily state or reflect those of the United States Government or the University of California, and shall not be used for advertising or product endorsement purposes.

This report has been reproduced directly from the best available copy.

Available to DOE and DOE contractors from the Office of Scientific and Technical Information P.O. Box 62, Oak Ridge, TN 37831

Prices available from (615) 576-8401, FTS 626-8401

Available to the public from the National Technical Information Service

U.S. Department of Commerce 5285 Port Royal Rd., Springfield, VA 22161

Work performed under the auspices of the U.S. Department of Energy by Lawrence Livermore National Laboratory under Contract W-7405-ENG-48. 
UCRL-LR-132566

Distribution Category UC-0

\title{
Laser-Plasma Interactions Relevant to Inertial Confinement Fusion
}

\author{
K. B. Wharton \\ (Ph.D. Dissertation)
}

November 1998

LAWRENCE LIVERMORE NATIONAL LABORATORY

University of California $\bullet$ Livermore, California $\bullet 94551$ 



\title{
UNIVERSITY OF CALIFORNIA
}

\section{Los Angeles}

\author{
Laser-Plasma Interactions Relevant \\ to Inertial Confinement Fusion
}

\begin{abstract}
A dissertation submitted in partial satisfaction of the requirements for the degree Doctor of Philosophy

in Physics
\end{abstract}

by

Kenneth Bradford Wharton

1998 
(C) Copyright by

Kenneth B. Wharton

1998 
The dissertation of Kenneth Bradford Wharton is approved.

\section{Steven Cowley}

John Dawson, Committee Co-Chair

Chan Joshi, Committee Co-Chair

Warren Mori

University of California, Los Angeles

1998 
This work is dedicated to my brother, Paul, for his courage and his spirit. 


\section{TABLE OF CONTENTS}

$\begin{array}{ll}\text { List of Figures } & \text { ix }\end{array}$

\begin{tabular}{ll} 
Acknowledgments & x \\
\hline
\end{tabular}

Publications $\quad$ X v

$\begin{array}{ll}\text { Abstract } & \text { xvii }\end{array}$

1: Introduction 1

1.1: Motivation 1

1.1.1: Fusion Power 1

1.1.2: Laser-Plasma Interactions 3

1.1.3: Outline 4

1.2: Plasmas 4

1.2.1: Basic Plasma Parameters 4

1.2.2: Plasma Waves 5

1.3: Short-Pulse Lasers $\quad 8$

1.4: Laser-Driven Fusion 10

1.4.1: Fusion Basics 10

1.4.2: Direct Drive 11

1.4.3: Indirect Drive 12

1.4.4: Fast Ignition $\quad 14$ 
2: Laser-Plasma Interations

2.1: Low Intensity Interactions $\left(I \lambda^{2}<10^{13} \mathrm{~W} \mathrm{~cm}^{-2} \mu \mathrm{m}^{2}\right) 16$

2.1.1: Pondermotive Force

2.1.2: Coupling Processes

2.1.3: Thomson Scattering

2.2: Mid-Intensity Interactions $\left(10^{13}<\mathrm{I} \lambda^{2}<10^{17} \mathrm{~W} \mathrm{~cm}^{-2} \mu \mathrm{m}^{2}\right)$

2.2.1: Three-Wave Instabilities

20

2.2.2: Saturation Mechanisms

2.2.3: Filamentation

2.2.4: Absorption Processes

2.3: High Intensity Interactions $\left(\mathrm{I} \lambda^{2}>10^{17} \mathrm{~W} \mathrm{~cm}^{-2} \mu \mathrm{m}^{2}\right)$

2.3.1: Relativistic Effects

2.3.2: Collisionless Absorption

3: Interactions Between Crossing Laser Beams

3.1.1: Previous Work

3.1.2: Relevance to Indirect Drive Fusion

3.1.3: Relevance to Direct Drive Fusion

3.2.1: Production and Diagnosis of the Flowing Plasma 32

3.2.2: Crossing Beams in the Flowing Plasma 35

3.2.3: Results

3.3: Analysis

3.3.1: Possibility of Pump Heating 
4: Measurements of Hot Electrons Produced by

High Intensity Laser-Solid Interactions

4.1: Background $\quad 44$

4.1.1: Previous Work 44

4.1.2: Single Electron Energy Deposition 46

4.1.3: Electron Beams in Matter 48

4.2: Methods $\quad 51$

4.2.1: Description of the 100TW Laser 51

4.2.2: Description of Experiment 53

4.2.3: ITS Modeling $\quad 56$

4.3: Results and Analysis $\quad 58$

4.3.1: Electron Energies and Conversion Efficiencies 58

4.3.2: Electron Directionality 60

4.3.3: Analysis $\quad 62$

5: Laser-Solid Interactions with the Petawatt Laser 66

5.1: Experimental Set-up 66

5.1.1: Description of Laser 66

5.1.2: Description of Experiment 68

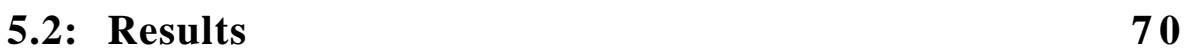

5.2.1: Data at 20ps pulse length 70

5.2.2: Data at 5ps pulse length 72

5.3: Analysis $\quad 74$ 
6: Implications for Inertial Confinement Fusion

6.1: Cross-Beam Effects

$\begin{array}{ll}\text { 6.1.1: Scaling to NIF } & 77\end{array}$

6.1.2: Resonance and k-matching in NIF 80

$\begin{array}{lr}\text { 6.1.3: Possible Solutions } & 83\end{array}$

6.1.4: Future Experimental Work 85

6.2: Fast Ignition $\quad 86$

6.2.1: General Implications $\quad 86$

6.2.2: Future Work $\quad 89$

$\begin{array}{lr}\text { 6.3: Summary } & 90\end{array}$

Appendix A: Energy Transfer in a General Three-Wave Resonance 93

A.1: Mathematical Model $\quad 93$

A.2: Resonant Energy Transfer $\quad 95$ A.3:

$\begin{array}{lll}\text { Steady-State Solution } & 97\end{array}$

$\begin{array}{lr}\text { A.4: Saturation } & 98\end{array}$

$\begin{array}{ll}\text { Bibliography } & \mathbf{1 0 0}\end{array}$ 


\section{LIST OF FIGURES}

1.1 Geometry of indirect-drive hohlraum planned for NIF

2.1 Stokes and anti-Stokes k-matching triangles

19

3.1 Geometry of indirect-drive hohlraum used on Nova

3.2 Thomson Scatter measurement of plasma flow velocity

3.3 Geometry of crossed-beam experiment 36

3.4 Transmission of Probe in crossed-beam experiment 38

3.5 Gain factor of Probe, plotted against position 39

3.6 Pump vs. Probe transmission fractions 40

4.1 100TW laser system diagram (front end) 51

4.2 Diagram of layered target for $K_{\alpha}$ measurements 53

4.3 Mean electron energy vs. temperature for relativistc electrons 57

4.4 $\mathrm{K}_{\alpha}$ signal from Aluminum targets (100TW) 58

4.5 $K_{\alpha}$ signal from Plastic and Copper targets 59

4.6 Sample curve fit for penumbral image data 61

4.7 Cone-angle measurements in plastic targets 62

5.1 Petawatt laser system diagram (final amplifiers) 67

5.2 Petawatt compressor chamber and target chamber diagram $\quad 67$

$5.3 \mathrm{~K}_{\alpha}$ signal from Aluminum targets (100TW and PW) 71

$5.4 \mathrm{~K}_{\alpha}$ signal from Aluminum targets (PW)

5.5 Conversion efficiency graph, as function of intensity 75

6.1 List of plasma parameters, comparing Nova to NIF 78 


\section{ACKNOWLEDGMENTS}

Having split my graduate student years equally between UCLA and Lawrence Livermore National Laboratory, I have numerous people to thank at both institutions.

Spanning both locations, however, I would first like to thank my advisor, Chan Joshi. His support, advice, and wisdom over the last five years have been invaluable; guiding me from the Mars lab to LLNL and even helping me hunt for a postdoc. If I go on to have a successful career, it will in no small part be due to Chan and his efforts as a superb advisor.

At UCLA, I would like to thank the rest of my thesis committee:

John Dawson, for inventing the beat-wave accelerator that got me started in experimental laser-plasma physics (not to mention nitrogen-doped buckyballs), and for his constant support as my advisor in the physics department;

Steve Cowley, for his role as my plasma-physics advisor and confidence in my research (although I'm actually not single-handedly carrying Livermore's fusion effort);

Warren Mori, for his feedback, friendship, and convincing theory of physics conferences that led me to overspend my per-diem nearly every time.

I would also like to express my thanks to many others at UCLA; in particular Chris Clayton for his patience with a novice experimentalist in the Mars lab and continuing support. Much of the practical lab experience I brought to Livermore was taught to me by Ken Marsh. George Morales, Frank Chen, and Chan Joshi are the excellent instructors who taught me about lasers and plasmas. Special thanks to my then-fellow graduate students Matt Everett, Amit Lal, and Dan Gordon for showing me the ropes, teaching me about laser-plasma interactions, and putting up with my bad jokes. Matt, Amit, and Dan, as well as Dave Blackwell, Rob Brogle, Pepe Davis, Patrick Muggli, Ritesh Narang and 
Kuo-Cheng Tseng were invaluable companions at lunch, conferences, and unmentioned virtual sporting events.

Huge amounts of praise must go to Maria Guerrero and Penny Lucky for helping me keep track of my very complicated situation, spanning two UCLA departments, a government laboratory, and my on-again, off-again status as an official student.

At LLNL, much thanks is due Bruce Hammel, for taking a chance on a random graduate student and continuing to support me through my thesis-writing period. Mike Key has supplied very useful leadership, guidance, and insight on the fast-ignitor side of things. My inclusion in Brian MacGowan's group has been very positive for me, and his support has been much welcomed. Important thanks should go to Mike Perry, for building the lasers with which it was easy to do interesting work.

On the short-pulse side of things, much thanks go to my fellow experimenters Yuri Zakarenkov, John Moody, Allan Offenberger and Jeff Koch, for helping make the 100TW experiments a success as well as teaching me about a wide variety of topics. The theoretical support of Steve Hatchett, Scott Wilks, and Max Tabak was arguably more important than the experiments themselves; many thanks are due for their help finding the meaning buried in the data. The laser support from Curtis Brown, Jeff Cardinal, John Miller, Steve Herman, Dee Pennington, Brent Stuart and Victor Yanovsky made these experiments possible and they deserve no end of praise. Further advice, assistance, and support was given by Tina Back, Chris Decker, Matt Everett, Seigfreid Glenzer, Bill Kruer, Barb Lasinski, Bruce Langdon, Luis da Silva, Russ Wallace, and the entire Nova Operations Crew, whose willingness to fit in "just one more 100TW shot" saved the facility from underuse. Also, gratitude is no doubt due to Joe Kilkenny and Mike Campbell for their behind-the-scenes support.

On the longer-pulse experiment(s), Bob Kirkwood deserves praise for guiding me and always pointing out both sides of most issues (even if it led me to change my mind 
several times!). John Moody and Seigfreid Glenzer were invaluable resources and tireless coworkers, not to mention that they had already set up most of the diagnostics I needed. Numerous conversations with Bedros Afeyan did much for my knowledge and understanding of plasma physics, and hopefully my work has reflected some of his guidance. Kent Estabrook was always there to do another LASNEX run, share his results, or just find out what was going on; thanks for being more than willing to help. Much gratitude to Bruce Cohen for his input, simulations, and patient theoretical advice. Further thanks go to Marc Blain, Gail Glendinning, Denise Hinkel and Dan Kalantar for patiently answering a large variety of questions; and Laurie Pinkerton who solved administrative and travel issues with seeming ease and a smile. And special thanks to Cameron Geddes for his companionship this last year and for smiling and nodding when I ranted about timereversed galaxies over lunch.

Outside the scientific world, I would like to thank many people for their love and friendship these six years. Most importantly, my wife Kate has been a constant source of happiness and encouragement over my entire graduate school experience (tropical islands notwithstanding). Maintaining sanity as a first-year grad student should also be attributed to John Hetts, my roommate at Hershey Hall, and the foozball table therein. Roommates Adam Bock and Tom Farquhar tagged in for sanity patrol the following two years, and all three of them deserve great thanks for being such excellent friends. Mike Keenan, Matt Kratter, Jeff Liu, Craig Parsons, Chris Regan, Alex Ryutov and Chacko Sonny were always there for fun, movies, and vodka, not necessarily in that order. And no thanks at all to Sid Meier.

My parents, Bill and Gwen Wharton, have been unfailing in their support. My father was the one who got me (and kept me) interested in physics, and his interest in my research has been much appreciated. My mother has always been there with needed advice, and her love and support have helped very much. I was very glad to have my 
wonderful sister, Ruth, in Los Angeles for a time -- even if she wasn't thrilled to be there. And a final note of gratitude goes to my brother, Paul; he is always in my thoughts, and this thesis is dedicated to him.

The author would like to thank the editors of Physical Review Letters for their permission to include excerpts from the following papers in this thesis: K.B. Wharton et al, "Experimental Measurements of Hot Electrons Generated by Ultraintense $\left(>10^{19} \mathrm{~W} / \mathrm{cm}^{2}\right)$ Laser-Plasma Interactions on Solid-Density Targets", Phys. Rev. Lett. 81, 822 (1998); and K.B. Wharton et al, "Observation of Energy Transfer between Identical-Frequency Laser Beams in a Flowing Plasma", Phys. Rev. Lett. 81, 2248 (1998). 


\section{VITA}

1970

Born, Seattle, Washington

1988-1989

Research Assistant

Argonne National Laboratory

Argonne, IL

1990

Research Assistant

Fermi National Laboratory

Batavia, IL

1992

B.S., Physics

Stanford University

Stanford, CA

1993-1995

Graduate Student Researcher

Electrical Engineering Department

University of California

Los Angeles, CA

1995-1998

Graduate Student Researcher

Lawrence Livermore National Laboratory

Livermore, CA 


\section{PUBLICATIONS}

K.B. Wharton, S. Hatchett, S.Wilks, M.Key, J.Moody, V.Yanovsky, A.A.Offenberger, B.Hammel, M. Perry, and C. Joshi; "Experimental Measurements of Hot Electrons Generated by Ultra-Intense $\left(>10^{19} \mathrm{~W} / \mathrm{cm}^{2}\right)$ Laser-Plasma Interactions on Solid Density Targets," Phys. Rev. Lett. 81, 822 (1998).

K.B. Wharton, R.K. Kirkwood, S.H. Glenzer, K.G. Estabrook, B.B. Afeyan, B.I. Cohen, J.D. Moody, and C. Joshi; "Observation of Energy Transfer Between Identical-Frequency Laser Beams in Flowing Plasmas," Phys. Rev. Lett. 81, 2248 (1998).

B.I. Cohen, B.F. Lasinski, A.B. Langdon, E.A. Williams, K.B. Wharton, R.K. Kirkwood, and K.G. Estabrook, "Resonant Stimulated Brillouin Interaction of Opposed Laser Beams in a Drifting Plasma", Phys.Plasmas 5, 3408 (1998).

M.H. Key, M.D. Cable, T.E. Cowan, K.G. Estabrook, B.A. Hammel, S.P. Hatchett, E.A. Henry, D.E. Hinkel, J.D. Kilkenny, J.A. Jock, W.L. Kruer, A.B. Langdon, B.F. Lasinski, R.W. Lee, B.J. MacGowan, A. MacKinnon, J.D. Moody, M.J. Moran, A.A. Offenberger, D.M. Pennington, M.D. Perry, T.J. Phillips, T.C. Sangster, M.S. Singh, M.A. Stoyer, M. Tabak, G.L. Tietbohl, M. Tsukamoto, K. Wharton, and S.C. Wilks, "Hot electron production and heating by hot electrons in fast ignitor research," Phys. Plasmas, 5, 1966 (1998).

J.A. Koch, C.A. Bach, C. Brown, K. Estabrook, B.A. Hammel, S.P. Hatchett, M.H. Key, J.D. Kilkenny, O.L. Landen, R.W. Lee, J.D. MOody, A.A. Offenberger, D. Pennington, M.D. Perry, M. Tabak, V. Yanovsky, R.J. Wallace, K.B. Wharton, and S.C. Wilks, "Time-resolved X-ray spectroscopy of deeply buried tracer layers as a density and temperature diagnostic for the fast ignitor," Laser and Particle Beams, 16, 225 (1998).

R.K.Kirkwood, B.J. MacGowan, D.S. Montgomery, B.B.Afeyan, W.L.Kruer, D.M.Pennington, S.C. Wilks, J.D.Moody, K.Wharton, C.A. Back, K.G. Estabrook, S.H.Glenzer, M.A.Blain, R.L. Berger, D.E.Hinkel, B.F. Lazinski, E.A.Williams, D. Munro, B.H.Wilde, and C. Rousseaux, "Observation of multiple mechanisms for stimulating ion waves in ignition scale plasmas," Physics of Plasmas, 4, 1800 (1997).

A. K. Lal, D. Gordon, K. Wharton, C. E. Clayton, K. A. Marsh, W. B. Mori, C. Joshi, M. J. Everett, and T. W. Johnston, "Spatio-temporal dynamics of the resonantly excited relativistic plasma wave driven by a $\mathrm{CO}_{2}$ laser," Physics of Plasmas 4, 1434 (1997).

B.C. Stuart, M.D. Perry, J. Miller, G. Tietbohl, S. Herman, J.A. Britten, C. Brown, D. Pennington, V. Yanovsky and K. Wharton, "125-TW Ti:sapphire/Nd:glass laser system", Optics Letters 22, 242 (1997). 
K. Wharton, Y. Zakharenkov, B. Hammel, S. Herman, J.Miller, J.Moody, A. Offenberger, D. Pennington, M. Perry, B. Stuart, C. Brown, V. Yanvosky, and S. Wilks, "Measurements of fast electrons produced in solid targets by laser intensities of $5 \times 10^{19}$ W/cm² (abstract)," Rev.Sci. Instr. 68, 847 (1997).

A. Lal, D. Gordon, K. Marsh, K. Wharton, C. Clayton, C. Joshi, "Exact Forward Scattering of a $\mathrm{CO}_{2}$ laser beam from a relativistic plasma wave by time resolved frequency mixing in $\mathrm{AgGaS}_{2}$," Rev. Sci. Instr. 68, 690 (1997).

D. Gordon, A. Lal, K. Wharton, C.E. Clayton, and C. Joshi, "Two Dimensional Cherenkov emission array for studies of relativistic electron dynamics in a laser plasma," Rev. Sci. Instr. 68, 358 (1997).

A. Lal, K. Wharton, D. Gordon, M. J. Everett, C. E. Clayton, and C.Joshi, "Measurements of the Beatwave Dynamics in Time and Space," proceedings of the PAC Conference, Dallas, TX, (May 1995).

M. J. Everett, A. Lal, D. Gordon, K. Wharton, C. E. Clayton, W. B.Mori, and C. Joshi, "Evolution of Stimulated Raman into Stimulated Compton Scattering of Laser Light via Wave-Breaking of Plasma Waves," Physical Review Letters 74, 1355 (1995). 


\title{
ABSTRACT OF THE DISSERTATION
}

\author{
Laser-Plasma Interactions Relevant \\ to Inertial Confinement Fusion
}

by

\author{
Kenneth Bradford Wharton \\ Doctor of Philosophy in Physics \\ University of California, Los Angeles, 1998 \\ Professor John Dawson, Co-Chair \\ Professor Chan Joshi, Co-Chair
}

\begin{abstract}
Research into laser-driven inertial confinement fusion is now entering a critical juncture with the construction of the National Ignition Facility (NIF) at Lawrence Livermore National Laboratory (LLNL). Many of the remaining unanswered questions concerning NIF involve interactions between lasers and plasmas. With the eventual goal of fusion power in mind, laser-plasma interactions relevant to laser fusion schemes is an important topic in need of further research.

This work experimentally addresses some potential shortcuts and pitfalls on the road to laser-driven fusion power. Current plans on NIF have 192 laser beams directed into a small cylindrical cavity which will contain the fusion fuel; to accomplish this the
\end{abstract}


beams must cross in the entrance holes, and this intersection will be in the presence of outward-flowing plasma. To investigate the physics involved, interactions of crossing laser beams in flowing plasmas are investigated with experiments on the Nova laser facility at LLNL. It was found that in a flowing plasma, energy is transferred between two crossing laser beams, and this may have deleterious consequences for energy balance and ignition in NIF. Possible solutions to this problem are presented.

A recently-proposed alternative to standard laser-driven fusion, the "fast ignitor" concept, is also experimentally addressed in this dissertation. Many of the laser-plasma interactions necessary for the success of the fast ignitor have not previously been explored at the relevant laser intensities. Specifically, the transfer of high-intensity laser energy to electrons at solid-target interfaces is addressed. $20-30 \%$ conversion efficiencies into forward-propagated electrons were measured, along with an average electron energy that varied with the type of target material. The directionality of the electrons was also measured, revealing an apparent beaming of the highest energy electrons. This work was extended to various intensities and pulse lengths and a relationship between conversion efficiency and laser intensity was deduced.

This work serves to advance knowledge of laser-plasma interactions relevant to a variety of laser-driven fusion schemes, and indicates directions for future research. These results should facilitate the success of NIF and other laser-fusion facilities. 


\section{Chapter 1}

\section{Introduction}

\subsection{Motivation}

\subsubsection{Fusion Power}

A star is drawing on some vast reservoir of energy by means unknown to us. This reservoir can scarcely be other than the subatomic energy which, it is known, exists abundantly in all matter; we sometimes dream that man will one day learn how to release it and use it for his service. The store is well-nigh inexhaustible, if only it could be tapped. [1]

This farsighted statement was made by Sir Arthur Stanley Eddington in 1920, shortly after the first demonstration of fusion in a laboratory by Sir Ernest Rutherford. While the promise of unlimited energy from fusion power was foreseeable even then, the struggle to realize this promise continued for the remainder of the century and is now continuing into the next.

After Eddington's realization, two years would pass before Irving Langmuir would even propose the term "plasma" to describe the state of the ionized matter in the sun, and it was not until 1929 that the sun's fusion reactions were in any way quantified. [2] Theoretical understanding of basic thermonuclear fusion reactions made great progress from that point onwards, and in 1952 fusion power was released in an uncontrolled explosion on the Eniwetok atoll in the Pacific Ocean. Edward Teller later 
wrote: "No sooner was it done, than every politician and every bureaucrat descended upon us saying, 'Now you must solve the problem of controlled fusion.'" [3,4]

At the end of the 20th century, that problem has yet to be solved. The promising technique of fusion by magnetic confinement $(\mathrm{MCF})$ has produced fusion yields close to "break-even", the point at which fusion energy output is equal to the input energy required.

An alternate approach to controlled fusion became possible with the invention of the laser in 1960.[5] In a matter of years, high power lasers were recognized as a possible mechanism with which to rapidly focus energy onto a small target and perhaps initiate fusion reactions. This technique, acronymed ICF for "inertial confinement fusion", requires much higher plasma densities than the corresponding longer-lived MCF devices due to a smaller size and much shorter plasma confinement time. And today it appears that the first machine capable of igniting a self-sustaining fusion burn may very well be an ICF device: the National Ignition Facility (NIF). This 1.2 billion dollar laser is currently scheduled to ignite a fusion reaction in 2005, nearly a full century after the fusion quest began.

The success of NIF and other planned Megajoule lasers is a crucial requirement for any practical ICF applications that may follow, but whether or not NIF will achieve ignition is far from certain. Numerous technological challenges are being faced to even build the world's first Megajoule laser facility; many of these difficulties have been overcome, but more remain. Once operational, further challenges will involve target fabrication, laser balance and alignment, and the ignition process itself. This dissertation aims to experimentally address some potential pitfalls and some potential shortcuts on the road to a confined fusion reaction. 


\subsubsection{Laser-Plasma Interactions}

In any laser-driven ICF scheme, laser-plasma interactions may comprise some of the most fundamental obstacles to ignition. Laser-plasma interactions have already constrained many details of the NIF design. For example, concern over high levels of energy loss from Stimulated Raman Scattering (SRS) and Stimulated Brillioun Scattering (SBS) - two fundamental laser-plasma instabilities — is the primary reason that NIF is being built with a $351 \mathrm{~nm}$ laser wavelength rather than the natural solid state laser wavelength of $1.06 \mu \mathrm{m}$. A fuller understanding of laser-plasma interactions is important to both achieve ignition and to design future ICF facilities.

Even if NIF ignites, however, a fusion-to-laser energy ratio of 10 is about optimum. Given that the electrical efficiency of the lasers will be less than $1 \%$, even a successful ignition would still not produce net energy, and higher gains would seem to require even more expensive facilities. However, a groundbreaking paper by Tabak et al. in 1994 [6] outlined the possibility of "fast ignition", a theoretical method to increase fusion gains to $\sim 1000$. This scheme would require a short-pulse ( 10ps) multi-kiloJoule laser to be used in conjunction with a more conventional ICF facility. Many questions about the feasibility of this technique remain, most of them concerning the nature of laser-plasma interactions at the enormous intensities required by such a scheme $\left(\sim 10^{20}\right.$ $\mathrm{W} / \mathrm{cm}^{2}$ ). Again, laser-plasma interactions are seen to be a key topic in the development of inertial confinement fusion.

Apart from relevance to ICF, the study of intense lasers in plasmas is fascinating it its own right as a testbed for basic physics. As the peak intensity of the interaction increases, so do the variety of possible laser-plasma processes. Keeping track of these complex interactions and forming a coherent picture of laser-plasma interactions is a rich and challenging undertaking. 


\subsubsection{Outline}

The central theme of this dissertation is the interaction of high power lasers with plasmas. Several aspects of laser-plasma interactions that are relevant to ICF will be discussed in detail.

The following chapters present the results and conclusions from two major experimental campaigns. Following the basic science overview in Chapters 1 and 2, Chapter 3 addresses the issue of energy transfer between laser beams in a flowing plasma. This is a crucial topic for symmetry considerations in NIF, as multiple laser beams will cross before they deposit their energy into the target. Chapter 4 moves on to the fast ignitor fusion scheme, and details the first comprehensive measurements of relevant laser-solid interactions at laser intensities above $10^{19} \mathrm{~W} / \mathrm{cm}^{2}$. Chapter 5 reports on the continuation of this work on the most powerful laser facility in the world: the Petawatt laser at LLNL. These experiments are ongoing, and the results are therefore less extensive than those in Chapter 4. Finally, Chapter 6 summarizes the experimental results, details their implications for ICF, and outlines new work that remains to be done.

\subsection{Plasmas}

\subsubsection{Basic Plasma Parameters}

A plasma is defined as a group of charged particles that behaves in a collective manner, and is a state of matter unlike any neutral-particle collection. In the field of high-intensity laser-produced plasmas there are few neutral particles; to an excellent approximation all particles are either positively charged ions or negatively charged electrons.

The plasma can be described in terms of a few fundamental parameters, all expressed in cgs units. The density of the plasma, $\mathrm{n}$, is expressed in particles per $\mathrm{cm}^{-3}$. The electron mass is denoted as simply $\mathrm{m}\left(\mathrm{m}=9.10910^{-28} \mathrm{~g}\right)$, while the electron charge 
is e (4.8 $10^{-10}$ statcoulombs in cgs units). The charge state of the ions is $\mathrm{Z}$, and the ion mass is M. If the each plasma species is in thermal equilibrium (Maxwellian velocity distribution), then temperatures can be assigned to the electrons $\left(\mathrm{T}_{\mathrm{e}}\right)$ and the ions $\left(\mathrm{T}_{\mathrm{i}}\right)$. If the velocity distribution is non-Maxwellian, the concept of temperature becomes less meaningful.

A key spatial scale for a plasma is the Debye length:

$$
\lambda_{D}=\left(\frac{k T_{e}}{4 \pi n e^{2}}\right)^{1 / 2}
$$

This is the characteristic distance over which the plasma electrons will shield out local electric fields. Therefore macroscopic neutrality will be maintained on scales longer than $\lambda_{\mathrm{D}}$. As a result, a true plasma can only be said to exist if the number of particles in a Deybe sphere, $n \lambda_{D}{ }^{3}$, is much greater than 1. A further consequence is that long wavelength structures in a plasma $\left(\lambda>>\lambda_{D}\right)$ are collective effects of the plasma, while short wavelength structures $\left(\lambda<<\lambda_{D}\right)$ will behave more like individual particles.

\subsubsection{Plasma Waves}

The collective nature of a plasma allows the existence of many types of waves. With no externally applied fields, electrostatic oscillations can take the form of highfrequency "electron waves" or lower-frequency "acoustic waves" (also known as "ion waves"). Electromagnetic oscillations (light waves) can also exist in a plasma.

If an initial perturbation is made in the electron density of a cold, field-free plasma, the bunched electrons will undergo simple harmonic motion at the plasma frequency:

$$
\omega_{p e}=\left(\frac{4 \pi n e^{2}}{m}\right)^{1 / 2}
$$


This frequency will therefore be a crucial parameter for electron plasma waves in finite-temperature plasmas. By substituting the ion charge and mass, an "ion plasma frequency" $\omega_{\text {pi }}$ can be found, but this frequency is much lower than $\omega_{\text {pe }}$ and does not significantly contribute to any plasma waves.

The so-called "normal modes" of a plasma with no imposed fields can be found by setting the externally applied charge and current to zero in Maxwell's equations. This condition forces the solutions to be self-consistent modes in which the plasma can oscillate, and therefore these will be the primary energy-bearing modes in a plasma. For a wave with a defined complex frequency $\omega=\operatorname{Re}(\omega)+i \operatorname{Im}(\omega)$ and k-vector $\mathbf{k}$, all wavedependent parameters scale as the real part of $\mathrm{e}^{(\mathrm{ik} \cdot \mathbf{x}-\mathrm{i} \omega \mathrm{t})}$, with an added phase. From this wave dependence and the assumption of no external fields, kinetic theory can express the normal modes of a neutral, field-free plasma by the following two equations:

$$
\begin{aligned}
& \left(1+\sum_{\alpha} \frac{\omega_{p \alpha}^{2}}{k^{2}} \bullet \int \frac{\partial F_{\alpha 0} / \partial u}{\omega /|k|-u} d u\right) \bar{E}_{k}=0 \\
& \left(1-\frac{k^{2} c^{2}}{\omega^{2}}-\sum_{\alpha} \frac{\omega_{p \alpha}^{2}}{\omega} \bullet \int \frac{F_{\alpha 0}}{\omega-|k| u} d u\right) \bar{E}_{\perp}=0
\end{aligned}
$$

In Eqns. 1.3 and 1.4, which must both hold for a normal mode of the plasma, the subscript $\alpha$ represents the various species in the plasma (electron, ions), and $\omega_{\mathrm{p} \alpha}$ is the species-dependent frequency from Eqn. 1.2 using the appropriate charge and mass. $E_{\mathbf{k}}$ is the electric field in the direction of the propagation of the wave, and $\mathrm{E}_{\perp}$ is the electric field perpendicular to $\mathbf{k}$. $\mathrm{F}_{\alpha_{\mathrm{o}}}(\mathrm{u})$ is the unmodified velocity distribution of the $\alpha$-species plasma, with its integral normalized to 1. For an isotropic Maxwellian (thermalized) distribution, with $\kappa$ as Boltzmann's constant, this is simply:

$$
F_{\alpha 0}(u)=\left(\frac{m_{\alpha}}{2 \pi \kappa T_{\alpha}}\right)^{1 / 2} \exp \left(\frac{-m_{\alpha} u^{2}}{2 \kappa T_{\alpha}}\right)
$$


Taken together, equations [1.3-5] determine the dispersion relation (the relation between $\omega$ and $\mathrm{k}$ ) for three fundamentally different plasma waves. For transverse waves $\left(E_{k}=0\right)$, the large term in Eqn. 1.4 must be zero, and therefore $\omega>k c>>k u$ for all $u$ where $\mathrm{F}_{\alpha \mathrm{o}}(\mathrm{u})$ is not negligible. This inequality allows the expansion of the integral, yielding to an excellent approximation:

$$
\omega^{2}=\omega_{p e}^{2}+k^{2} c^{2}
$$

This is the dispersion relation for electromagnetic waves in a plasma; these waves must be at or above the plasma frequency. Therefore, an electromagnetic wave with frequency $\omega$ will not be able to propagate through a plasmas above the "critical density":

$$
n_{c r}=\frac{m \omega^{2}}{4 \pi e^{2}}
$$

Further normal modes of the plasma are found to be longitudinal waves, where now $\mathrm{E}_{\perp}=0$, and the large term in Eqn. 1.3 must be set to zero. This equation has a highand low-frequency solution. Like the electromagnetic wave, the high-frequency mode satisfies $\omega>\omega_{\text {pe }}$, and the same expansion of the integral can be made. Assuming both $\mathrm{k} \lambda_{\mathrm{D}}$ and $\operatorname{Im}(\omega) / \operatorname{Re}(\omega)$ are small, the dispersion relation (for the real part of the frequency) is:

$$
\omega^{2}=\omega_{p e}^{2}+\frac{3 \kappa T_{e}}{m} k^{2}
$$

This is the dispersion relation for electron plasma waves, or Langmuir waves. In the low-frequency limit, the ion terms cannot be neglected and a separate expansion must be made. Integration then yields a least-damped root where:

$$
\omega=\left(\frac{Z \kappa T_{e}}{M\left(1+k^{2} \lambda_{D}^{2}\right)}+\frac{3 \kappa T_{i}}{M}\right) k \equiv c_{s} k
$$

The "sound speed" of the plasma, $\mathrm{c}_{\mathrm{s}}$, is defined in Eqn. 1.9. However, this is only true in a stationary plasma; a flowing plasma will affect this dispersion relation. This is a very small correction for the electromagnetic and Langmuir waves because they 
have a very large phase velocity $(\omega / \mathrm{k})$, which a plasma flow velocity $\left(\mathbf{v}_{\mathrm{f}}\right)$ typically does not approach. But given that $\mathbf{v}_{\mathrm{f}}$ can be comparable to $\mathrm{c}_{\mathrm{s}}$, a correction must be made in the dispersion relation for ion waves:

$$
\omega=c_{s}|\mathbf{k}|+\mathbf{v}_{\mathbf{f}} \cdot \mathbf{k}
$$

This is the dispersion relation for ion waves in a plasma. Both Langmuir waves and ion waves also have imaginary frequency components that exponentially damp the wave through a mechanism known as Landau damping. For an ion wave, this turns out to be:

$$
\operatorname{Im}(\omega)=-\operatorname{Re}(\omega)\left(\frac{\pi}{8}\right)^{1 / 2} \theta(3+\theta)^{1 / 2} e^{-(3+\theta) / 2}[1.11]
$$

Here, $\theta=\mathrm{ZT}_{\mathrm{e}} / \mathrm{T}_{\mathrm{i}}$. This represents the loss of wave energy to ions that have a velocity close to the phase velocity of the ion wave. This term is not directly affected by plasma flow, as the phase velocity of the ion wave is always $c_{s}$ in the frame of the plasma.

The three dispersion relations in Eqns. [1.6], [1.8], and [1.10] represent all normal modes of a field-free plasma. Non-normal modes can also exist in a plasma, but they must have a source charge and/or current driven by outside factors or other waves. The issue of both normal and non-normal modes being driven by other plasma waves will be discussed in Chapter 2.

\subsection{Short-Pulse Lasers}

High-power, short pulse lasers are unique instruments because of their ability to produce immense localized electric fields that oscillate at a particular frequency. By increasing the peak intensity of a laser, new regimes of physics become accessible. However, there are several fundamental barriers that limit the peak theoretical power of any laser system. One limit is the pulse duration of the laser; compressing the same 
amount of energy into a shorter pulse will yield a higher power laser, but the following relationship must continue to hold:

$$
\Delta f_{r m s} \Delta t_{r m s}>0.5
$$

Here $\Delta \mathrm{f}_{\text {rms }}$ is the root-mean-square bandwidth of the spread in the laser's frequencies, and $\Delta \mathrm{t}_{\mathrm{rms}}$ is the root-mean-square of the pulse duration. Therefore a shortpulse laser requires a large bandwidth. However, amplifying a large-bandwidth short pulse creates additional constraints. One effect is gain-narrowing, caused by the tendency of amplifiers to have different amplifier efficiencies at different frequencies. The net result can be to primarily boost the energy in a range of frequencies smaller than $\Delta \mathrm{f}_{\mathrm{rms}}$, which (according to Eqn. 1.12) has the unwanted effect of increasing $\Delta \mathrm{t}_{\mathrm{rms}}$. This problem can be somewhat alleviated by using different types of amplifiers in a chain. Another scheme has been developed to selectively attenuate the spectrum between each amplifier pass, allowing the amplification of <20fs pulses. [7]

Another barrier to high-power laser systems is amplifier damage. A function of intensity, damage can be alleviated by increasing the physical diameter of the amplifier system, and thereby lowering the average intensity of the laser in the amplifier material. This technique is capable of producing several kilojoules in a 1ns pulse, as done with large $46 \mathrm{~cm}$ solid-state glass amplifiers at the Nova facility at Lawrence Livermore National Laboratory (LLNL). However, shorter pulses increase the damage threshold, and a method known as "chirped pulse amplification" (CPA) is required to raise peak laser power much above a terawatt. [8]

CPA is accomplished by utilizing the bandwidth that is present in short-pulse lasers. Although Eqn. 1.12 represents the shortest possible laser pulse for a given bandwidth, longer pulses are achievable by "chirping" the laser. A chirped pulse is one in which the different frequency components are separated in time, thereby creating a longer, lower-intensity pulse. Using diffraction gratings which can create different path 
lengths for each frequency component, a sub-ps pulse can be stretched out, amplified, and then recompressed. This avoids the damage threshold of the amplifier, although now the final compression grating must handle the full laser intensity. Because of this, large diffraction gratings are required to avoid damage.

Once compressed, the final focus of the laser beam will determine the peak intensity. The smallest theoretical focal spot diameter (measured between the 1/e points of the peak field) occurs for the $\mathrm{TEM}_{00}$ mode (Gaussian profile) of a laser beam:

$$
\sigma_{1 / e} \approx \frac{4}{\pi} f_{\#} \lambda
$$

Here $f_{\#}$ is the $f$-number (diameter/focal length) of the final focusing optic and $\lambda$ is the wavelength. This ideal focal spot is known as "diffraction limited". In practice, most laser systems above 1 Joule of energy can at best reach 2-3 times diffraction limited focal spots.

\subsection{Laser-Driven Fusion}

\subsubsection{Fusion Basics}

Nuclear reactions can yield energy by tapping into the binding energy between nucleons. Like gravitational energy, nuclear binding energy is negative and therefore increasing binding strength leads to a release of energy.

The average binding energy per nucleon generally increases as nuclei get larger, reaching a maximum of $\sim 9 \mathrm{MeV} /$ nucleon for ${ }^{62} \mathrm{Ni}$. Larger nuclei have progressively less binding energy per nucleon. Therefore, fission reactions of heavy elements will release energy, as will fusion reactions of light elements. The weakest binding energy of any

stable multi-component nucleus is the $\sim 1 \mathrm{MeV} /$ nucleon of ${ }^{2} \mathrm{H}$ (commonly known as Deuterium, or D). The radioactive nucleus ${ }^{3} \mathrm{H}$ (Tritium, or $\mathrm{T}$ ) also has a low binding energy ( $2.5 \mathrm{MeV} /$ nucleon$)$. In contrast, ${ }^{4} \mathrm{He}$ is very stable and has a strong binding 
energy of $\sim 7 \mathrm{MeV} /$ nucleon. Therefore fusion reactions that transmute weakly bound nuclei into ${ }^{4} \mathrm{He}$ will be energetically favorable.

The highest cross-section of any useful fusion reaction is for the reaction:

$$
\mathrm{D}+\mathrm{T} \Rightarrow{ }^{4} \mathrm{He}(3.5 \mathrm{MeV})+{ }^{1} \mathrm{n}(14.1 \mathrm{MeV})
$$

For a thermal collection of $\mathrm{D}$ and $\mathrm{T}$, the cross section is maximum at $\mathrm{T}_{\mathrm{i}}=\sim 43 \mathrm{keV}$. For a self-sustaining fusion burn (also known as ignition) the fusion products from Eqn. [1.14] must heat the fuel close enough to this temperature such that further fusion reactions can take place. For a given fusion cross section, the reaction rate density scales as the fuel density squared, and therefore large densities are a crucial factor for an energy-producing fusion reaction. Because of this density scaling, the attempt to achieve ever-higher fuel compression has driven much of previous ICF research.

\subsubsection{Direct Drive}

The first, and most obvious method of achieving controlled fusion with lasers is to directly heat and compress a fuel pellet with one or more laser beams. Because a higher density plasma will have a shorter ion-ion collision time (compared to the disassembly time of the plasma), using lasers to compress the fuel pellet is crucial.

Because compression is so important, the preferred direct-drive scheme is to start with a spherical Deuterium-Tritium (DT) fuel pellet and irradiate it uniformly on all sides. The ablating outer fuel will both heat and compress the pellet, creating a high temperature (but low-density) main fuel that surrounds a high-density (but lower temperature) spot in the center. The two regions are in pressure equilibrium, so this is known as an "isobaric" implosion. The implosion will ideally create the appropriate conditions in the central spot $\left(\mathrm{T}_{\mathrm{i}}>5 \mathrm{keV}\right.$, density $\mathrm{x}$ diameter $\left.>0.3 \mathrm{~g} / \mathrm{cm}^{2}\right)$ such that it will undergo thermonuclear fusion and ignite the pellet. [6] 
In practice, achieving a spherically symmetric implosion with laser beams is extremely difficult. Much progress has been made over the years, however, and the most energetic laser in the world today -- the 60-beam Omega facility at the University of Rochester Laboratory for Laser Energetics -- has been designed around direct-drive principles. The National Ignition Facility is also being designed with a direct-drive option.

\subsubsection{Indirect Drive}

One way to relax the symmetry constraints is to indirectly heat the pellet with secondary x-rays. In the indirect drive scheme, the fuel pellet is mounted inside of a high-Z enclosure, called a hohlraum. Hohlraums can be cylindrical with two Laser Entrance Holes (LEHs), or tetrahedral with four LEHs, and are typically made of gold. Multiple laser beams pass through each LEH and strike the inside of the hohlraum wall (while avoiding the pellet), depositing their energy into x-rays. Nonuniformities in the laser profiles are smeared out by the x-ray spectrum, which in turn can heat and compress the pellet in the center. While this is a less efficient technique than direct-drive, the inherent smoothing benefit has led to the primary design of the NIF as an indirect-drive machine.

While indirect-drive symmetry is easier to achieve from a laser engineering standpoint, it still imposes many constraints. Multiple laser beams are required to maximize the symmetry on the inside of the hohlraum; NIF will have 192 beams. Figure 1.1 shows the current geometry for an indirect drive target on NIF, with each 4-beam cluster represented as a single beam. One concern for indirect drive is maintaining symmetry over time; as the gold wall of the hohlraum expands inward, the distribution of the x-ray radiation can change substantially. To counter this effect, NIF hohlraums are likely to be filled with a low-Z gas $(\mathrm{H}$ and $\mathrm{He})$ which will hold back the expansion of the 


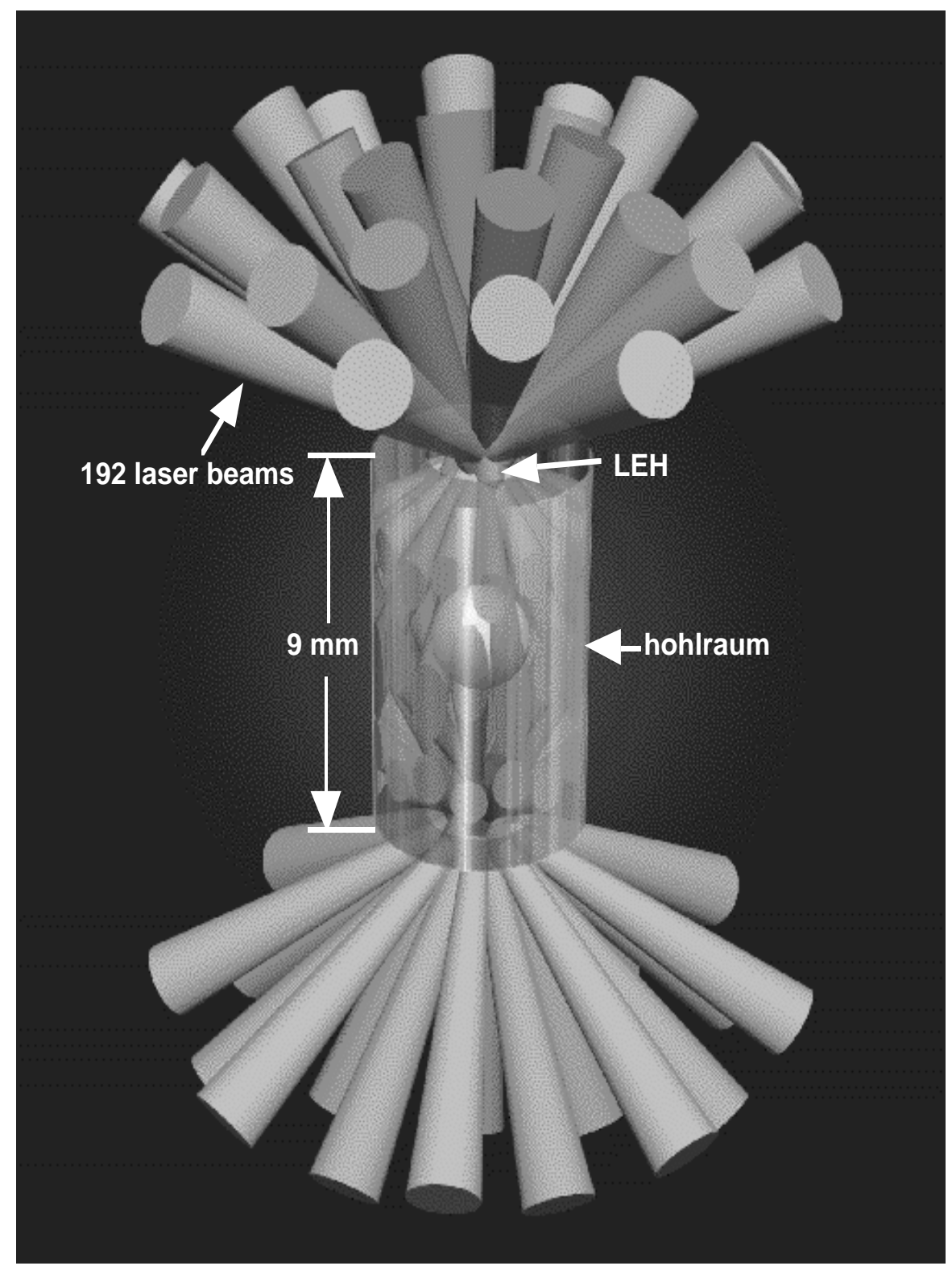

Figure 1.1. Shown is the geometry of an indirect-drive hohlraum in the planned National Ignition Facility. The fuel pellet sits at the center of the hohlraum. Each 4-beam cluster is represented as a single f/8 beam. All beams enter the hohlraum at either 23.5, 30, 46.5, or 50 degrees to the hohlraum axis.

wall. However, this will increase the density of the plasma inside the hohlraum, which may lead to additional laser-plasma interactions. 
Another distinction from direct-drive arises because the hohlraum (particularly a cylindrical hohlraum) is far from spherical; the distance from the pellet to the gold wall varies with position. Because of this geometrical asymmetry, the laser beams that hit the inside of the hohlraum farther from the pellet must be more intense to make up for the added distance. Together, these constraints on symmetry may force an ignition target into a parameter space where certain laser-plasma instabilities become important. This possibility will be explored further in Chapter 3.

\subsubsection{Fast Ignition}

Both of the above techniques call for an isobaric ignition of the fuel pellet. This will not lead to a very efficient fusion burn because the pressure balance constrains the hotter plasma on the inside to be correspondingly less dense than the cooler plasma on the outside, according to the equation of state of DT fuel. This leads to a less efficient burn of the outer fuel, and gives correspondingly sub-optimal energy gains, no matter how perfect the implosion.

An alternative to these schemes is an "isochoric" ignition, with the fuel at a constant density and out of pressure equilibrium because of a "hot spot" which initiates

the fusion burn. This type of pellet implosion would require less total energy to achieve; the difficulty lies in igniting the pellet by quickly depositing energy in a hot spot before pressure equilibrium can reestablish itself.

In 1994 Tabak et al. [6] proposed that this hot spot could be created with an extremely high power laser. This could occur in the following chain of events:

(I) A DT pellet is directly imploded by several kJ-energy laser beams of a few ns pulse duration. 
(II) A moderately high-intensity "pre-pulse" laser beam will strike the pellet from one side, forming a hollow plasma channel by means of pondermotive forces and relativistic filamentation (see Chapter 2 for more details).

(III) A very high intensity (multi $\mathrm{kJ}$ in $\sim 10 \mathrm{ps}$ ) laser pulse propagates through the hollow channel and deposits a large fraction of its energy into $\sim 1 \mathrm{MeV}$ electrons at the surface of critical plasma density.

(IV) These hot electrons propagate into the overdense plasma and heat the DT ions, igniting a localized fusion burn.

(V) This fusion burn releases enough energy to ignite the rest of the compressed fuel pellet.

This remains a speculative scenario, primarily because the mechanisms involved in steps (II) and (III) have not been experimentally addressed at the relevant intensities. Chapters 4 and 5 will present original work that experimentally addresses the laserelectron conversion required by step (III). Chapter 6 will also address the future possibilities for fast ignitor fusion. 


\section{Chapter 2}

\section{Laser-Plasma Interactions}

\subsection{Low Intensity Interactions $\left(\mathrm{I} \lambda 2<1013 \mathrm{~W} \mathrm{~cm}^{-2} \mu \mathrm{m}^{2}\right)$}

\subsubsection{Pondermotive Force}

Electrons in the electromagnetic field of a laser will undergo sinusoidal oscillations with a peak velocity of:

$$
v_{\text {osc }}=\frac{e E_{o}}{m \omega}
$$

Here $E_{0}$ is the peak electric field at the location of the electrons. Note that this is proportional to $\left(\mathrm{I} \lambda^{2}\right)^{1 / 2}$, where $\mathrm{I}$ is the intensity of the laser and $\lambda$ is the wavelength.

Therefore this is a key parameter for determining the strength of any laser-plasma interaction.

In the above equation, the electrons are not in a single location during the course of the oscillation and therefore in a realistic system they will sample a range of peak electric fields $\left(\mathrm{E}_{\mathrm{o}}\right)$. Any spatial variation in the electric field will prevent the oscillation from being perfectly sinusoidal, and the result will be a time-averaged force on the electrons given by:

$$
\mathbf{F}_{p}=-\frac{e^{2}}{4 m \omega} \nabla\left\langle\mathbf{E}^{2}(\mathbf{x})\right\rangle
$$

$F_{p}$ is known as the pondermotive force, and tends to push electrons away from regions of high fields (averaged over many oscillations). When the ions follow to maintain charge neutrality, a density perturbation is created. This equation holds for any 
oscillating E-field in a plasma, including those made by electron plasma waves, so long as the force is measured on a long timescale compared to the period of the oscillation.

\subsubsection{Coupling Processes}

Suppose there are two waves in a plasma, the first with frequency and wavenumber $\left(\omega_{0}, \mathbf{k}_{\mathbf{0}}\right)$, and the second with $\left(\omega_{1}, \mathbf{k}_{\mathbf{1}}\right)$ such that $\omega_{0} \geq \omega_{1}$. The electric fields associated with these waves will sum to:

$$
\mathbf{E}(\mathbf{x}, t)=\operatorname{Re}\left[\mathbf{E}_{\mathbf{0}} e^{i\left(\mathbf{k}_{0} \bullet \mathbf{x}-\omega_{0} t+\phi_{0}\right)}+\mathbf{E}_{1} e^{i\left(\mathbf{k}_{1} \bullet \mathbf{x}-\omega_{1} t+\phi_{1}\right)}\right]
$$

Here $\phi_{0}$ and $\phi_{1}$ are the initial phases of the two waves, and $\mathbf{E}_{\mathbf{0}}$ and $\mathbf{E}_{\mathbf{1}}$ are their respective amplitudes. The direction of $\mathbf{E}$ can either be parallel to $\mathbf{k}$ (Langmuir, acoustic waves) or perpendicular (electromagnetic waves), and the two k-vectors can be at arbitrary angles to each other.

The pondermotive force produced by this electric field will have four exponential terms, calculated by inserting [2.3] into [2.2]. Two of these terms will simply oscillate at the frequency and wavenumber of the original two waves, but there will be two additional terms with a frequency and k-number given by:

$$
\begin{aligned}
& \omega_{s}=\omega_{0}-\omega_{1} ; \mathbf{k}_{s}=\mathbf{k}_{0}-\mathbf{k}_{1} \\
& \omega_{a s}=\omega_{0}+\omega_{1} ; \mathbf{k}_{a s}=\mathbf{k}_{0}+\mathbf{k}_{1}
\end{aligned}
$$

These are the frequency- and k-matching conditions for Stokes coupling (Eqn. [2.4]) and anti-Stokes coupling (Eqn. [2.5]), analogous to conservation of energy ( $\hbar \omega)$ and momentum $(\hbar k)$ in quantum mechanics. While this simple derivation only applies for high-frequency waves, these are also the correct matching equations for processes involving ion acoustic waves. When one of the waves is in this low-frequency regime, the coupling mechanism is no longer the pondermotive force but rather a source current term $\left(\mathrm{j}_{\mathrm{s}}\right)$ that arises from the low frequency density fluctuations $\left(\mathrm{n}_{\mathrm{ia}}\right)$ and the higher frequency velocity fluctuations $\left(j_{\mathrm{s}}=-e \mathrm{n}_{\mathrm{ia}} \mathrm{v}_{\mathrm{OSc}}\right)$. 
Neither of the above-described couplings have a threshold wave amplitude, and this process can therefore drive a third wave in arbitrarily low intensity laser-plasma interactions. For example, two laser beams $\left(\omega_{0}, \mathbf{k}_{\mathbf{0}}\right)$ and $\left(\omega_{1}, \mathbf{k}_{\mathbf{1}}\right)$ can create source terms by which non-normal modes of the plasma may be driven at both $\left(\omega_{\mathrm{s}}, \mathbf{k}_{\mathbf{s}}\right)$ and $\left(\omega_{\mathrm{as}}, \mathbf{k}_{\mathbf{a s}}\right)$. If either of these driven waves is a normal mode of the plasma, the response will be much stronger and more energy will couple into the driven mode.

\subsubsection{Thomson Scattering}

In a very close analogy to the two-laser interaction discussed above, a single laser beam $\left(\omega_{0}, \mathbf{k}_{\mathbf{0}}\right)$ can interact with a plasma wave $\left(\omega_{1}, \mathbf{k}_{\mathbf{1}}\right)$ by a process known as Thomson scattering. As before, if one of the coupled modes is also a normal mode of the plasma, a significant amount of energy can be scattered into this mode. In the case of Thomson scattering, this third mode is defined to be an electromagnetic wave with its wavenumber and frequency shifted according to Eqn. 2.4 or Eqn. 2.5.

Because of this frequency shift, Thomson scattering is an excellent diagnostic of the various waves in a plasma. By spectrally and temporally resolving the scattered light in a particular direction, one can reconstruct the plasma waves $\left(\omega_{1}, \mathbf{k}_{1}\right)$ from which the light was scattered. This can be done both for strongly driven waves and loweramplitude thermal waves (driven by thermal fluctuations) provided the intensity of the original probe laser is sufficiently high. This will be an important diagnostic for the experiment described in Chapter 3.

When a Thomson scatter diagnostic is k-matched to one thermal wave, it is usually also k-matched to a second wave with a k-vector directed opposite to the first. This is shown graphically in Figure 2.1. Suppose Eqn $2.4\left(\mathbf{k}_{\mathbf{s}}=\mathbf{k}_{\mathbf{0}}-\mathbf{k}_{\mathbf{1}}\right)$ signifies the Thomson scatter k-matching conditions (Stokes coupling), with $\mathbf{k}_{\mathbf{0}}$ representing the kvector of original laser, $\mathbf{k}_{\mathbf{1}}$ as the thermal plasma wave, and $\mathbf{k}_{\mathbf{s}}$ as the scattered light. 


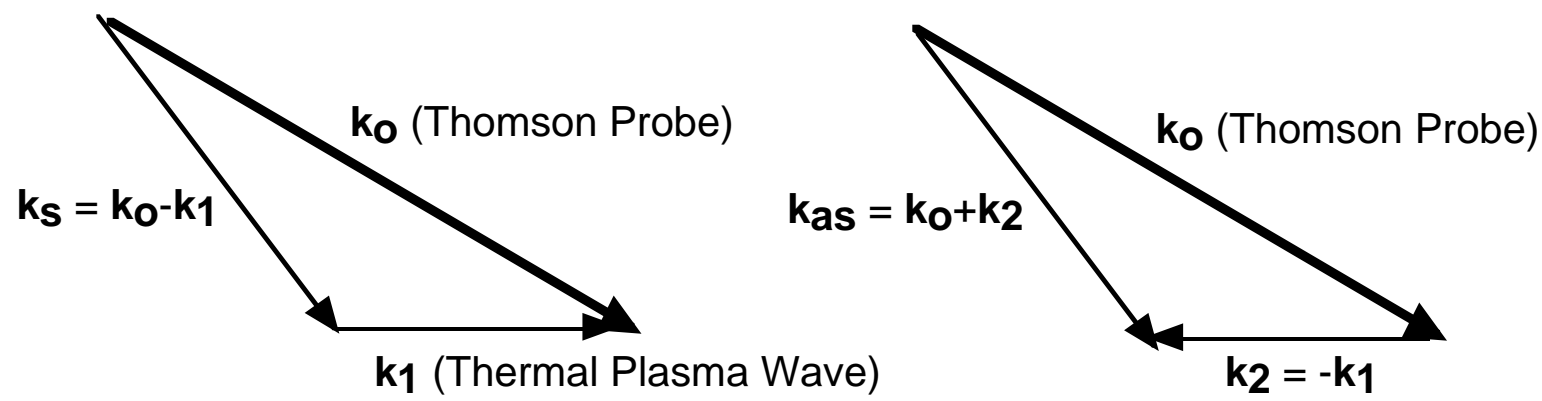

Figure 2.1. Two k-matching triangles that demonstrate why a detector positioned to measure the Thomson scattered Stokes wave $\left(\mathrm{k}_{\mathrm{S}}\right)$ from a thermal plasma wave $\left(\mathrm{k}_{1}\right)$ will also measure the scattered Anti-Stokes wave $\left(\mathrm{k}_{\mathrm{as}}\right)$ from another thermal plasma wave in the opposite direction $\left(\mathrm{k}_{2}\right)$. For scattering off ion acoustic waves, the frequency difference between the two scattered signals is small, and the triangles will be nearly the same shape.

Antistokes coupling (Eqn 2.5) off another plasma wave $\left(\omega_{2}, \mathbf{k}_{2}\right)$ would only be relevant if the scattered light $\left(\mathbf{k}_{\mathbf{a s}}=\mathbf{k}_{\mathbf{0}}+\mathbf{k}_{\mathbf{2}}\right)$ reaches the detector set up to measure $\mathbf{k}_{\mathbf{s}}$, or if $\mathbf{k}_{\mathbf{a s}}=\mathbf{k}_{\mathbf{s}}$. This could happen for a second thermal plasma wave if $\mathbf{k}_{\mathbf{2}}=-\mathbf{k}_{\mathbf{1}}$. Given $\mathbf{k}_{\mathbf{2}}, \omega_{2}$ is then determined from the appropriate dispersion relation.

However, taking frequency-matching into account in Eqns. [2.4] and [2.5] (recall that for Eqn. [2.5] the "1" subscripts are now "2"), $\omega_{\mathrm{as}}-\omega_{\mathrm{S}}=\omega_{1}+\omega_{2}$. If this frequency difference $\left(\omega_{\mathrm{as}}-\omega_{\mathrm{S}}\right)$ is large, then $\mathbf{k}_{\mathbf{a s}}$ can deviate strongly from the original $\mathbf{k}_{\mathbf{s}}$ (according to the electromagnetic dispersion relation from Eqn 1.6). But if $\omega_{1}$ and $\omega_{2}$ are both small compared to the laser frequency $\omega_{0}$ (ion acoustic waves, for example) then this is not a large effect. As a result, spectral analysis of Thomson scattered light off of thermal ion waves appears as two lines in frequency space, separated by $\omega_{1}+\omega_{2}=2 \omega_{\text {ia }}$. In a stationary plasma, these lines are symmetrical around the central laser frequency of $\omega_{0}$. 


\subsection{Mid-Intensity Interactions $\left(10^{13}<\mathrm{I} \lambda^{2}<10^{17} \mathrm{~W} \mathrm{~cm}^{-2} \mu \mathrm{m}^{2}\right)$}

\subsubsection{Three-Wave Instabilities}

As the peak intensity of a laser increases, so do the possible types of laser-plasma interactions. When the electric field $\mathrm{E}_{0}$ becomes large enough, the above-described Stokes and anti-Stokes coupling can occur between a laser and the background (thermal) waves of a plasma. Using the earlier analogy between $\omega$-matching and energy conservation, one can understand why Stokes coupling is more likely than anti-Stokes in the case of a single pump; energy flows from the pump laser $\left(\omega_{0}, \mathbf{k}_{\mathbf{0}}\right)$ into two lowerfrequency "daughter waves", $\left(\omega_{1}, \mathbf{k}_{1}\right)$ and $\left(\omega_{\mathrm{s}}, \mathbf{k}_{\mathbf{s}}\right)$. Stokes coupling between the pump and either daughter wave will drive the other daughter wave, allowing a feedback mechanism that can drive both daughter waves up from the thermal fluctuations initially present in the plasma. This can be a significant drain of energy from the original laser beam, and therefore these processes are a concern in laser-fusion relevant experiments.

This three-wave process forms the basis for many laser instabilities, known by different names depending on the form of the daughter waves. Stimulated Raman Scattering (SRS) occurs when a light wave is coupled to another light wave and a Langmuir wave. Stimulated Brillioun Scattering (SBS) is like SRS but with an ion acoustic wave instead of a Langmuir wave. Both of these processes can occur in a variety of geometries, but usually are strongest when the scattered light is backscattered, or propagating in the opposite direction from the pump.

While SBS and SRS generally require only one pump beam, two beam interactions can also drive such processes. As discussed in section 2.1.2, when the plasma beat-wave (Stokes-coupled mode) is a normal mode of the plasma, the driven wave can have a very strong response known as nonlinear mixing [9]. 
Mathematical details of three-wave resonant interactions are given in Appendix A. The simplified equations in the appendix show that in a low-damping limit, energy will be exchanged between the three waves continuously, with no time-averaged net energy transfer. When one of the three waves (typically a plasma wave) is strongly damped, the higher-frequency undamped wave will transfer energy to the lowerfrequency undamped wave. This interaction will also transfer energy to the damped plasma wave, and thereby heat the plasma.

When the driving waves themselves are intense, simple linear theory predicts extremely large wave amplitudes for the driven wave and correspondingly large amounts of energy transfer between the beams. For the example of two laser beams (both with wavelengths of $\left.\sim \lambda_{0}\right)$ that resonantly drive an ion acoustic beat-wave $\left(\omega_{i \mathbf{i a}}, \mathbf{k}_{\mathbf{i a}}\right)$, the intensity enhancement of the lower-frequency laser beam can be derived as $\exp (\mathrm{Q})$ where [10]:

$Q_{\max }=\frac{\pi}{2} \frac{n}{n_{c r}}\left(\frac{v_{o s c}^{2}}{v_{\text {the }}^{2}}\right) \frac{k_{i a} c_{s}}{\operatorname{Im}\left(\omega_{i a}\right)} \frac{L}{\lambda_{0}} \frac{1}{\left(1+3 T_{i} / Z T_{e}\right) \cos \theta_{s}}$

Here, $\mathrm{v}_{\text {the }}$ is the electron thermal velocity, $\left(2 \kappa \mathrm{T}_{\mathrm{e}} / \mathrm{m}\right)^{1 / 2}$, and $\kappa$ is the Boltzmann constant. $\mathrm{L}$ is the interaction length, $\theta_{\mathrm{s}}$ is the angle between the incident laser beams, and the other terms have been defined in Chapter 1. This is the exponential gain over a length L for a homogeneous plasma at perfect resonance. Laser pump depletion is neglected here (see Appendix A), which means that a large value for Q implies near total transfer of energy from the higher-frequency to the lower-frequency beam. 


\subsubsection{Saturation Mechanisms}

Apart from pump depletion, there are other mechanisms which can limit energy transfer to less than the gain indicated by linear theory. In the above example of two lasers driving an ion wave, any nonlinear effect that limits the growth of the resonant ion wave will in turn limit the energy transfer between the lasers. While ion Landau damping is included in Eqn. 2.6 in the form of the damping rate $\operatorname{Im}\left(\omega_{\mathrm{ia}}\right)$, plasma inhomogenieties are not.

Inhomogenieties in the plasma (such as a density perturbation) can shift the frequency of the resonant plasma wave away from the beat-frequency of the driving waves. This will diminish the resonant plasma response and will thereby limit any transfer of energy. The half-width of the resonance in frequency space is given by $\operatorname{Im}(\omega) / 2[10,11]$ : this is the scale on which inhomogenieties become important.

For the above example of a resonant ion wave, the important inhomogenieties are the ones that shift the ion acoustic frequency $\omega_{\text {ia }}$ (given by Eqn [1.10]) away from the frequency difference of the lasers by an amount $\operatorname{Im}\left(\omega_{\mathrm{ia}}\right) / 2$. This can occur as a result of fluctuations in plasma flow velocity $\left(\mathrm{v}_{\mathrm{f}}\right)$ or either component of the plasma temperature $\left(\mathrm{T}_{\mathrm{e}}, \mathrm{T}_{\mathrm{i}}\right)$ which can then alter the sound speed $\left(\mathrm{c}_{\mathrm{s}}\right)$.

A more complicated inhomogeniety can occur when the electron distribution function $\mathrm{F}_{\mathrm{e} 0}(\mathrm{u})$ is shifted from the simple Maxwellian described in Eqn [1.5]. One can write a more general distribution function by replacing the $\exp \left(-\mathrm{u}^{2}\right)$ dependence in Eqn [1.5] with a $\exp \left(-\mathrm{u}^{\mathrm{n}}\right)$ dependence, while at the same time changing the various constants to force normalization and allow a new definition of "temperature". A value of $n=2$ corresponds to a Maxwellian distribution, but other values of $\mathrm{n}$ are possible as well. When $\mathrm{n}$ increases (for example, via the preferential loss of higher energy electrons from a given region of plasma) the distribution becomes more "flat-topped", and electronelectron collisions become entirely negligible at $n=5$. It has been shown $[12,13]$ that a 
shift in this exponent can lead to a corresponding shift of the ion acoustic frequency, among other plasma parameters. Therefore modified distribution functions are another type of inhomogeniety that can detune (or possibly enhance) a resonant process.

Intensity fluctuations of the driving lasers can also affect the energy transfer in resonant processes by changing $\mathrm{v}_{\text {osc }}$ in Eqn. [2.6]. All of these effects can build off of each other, with intensity fluctuations in turn creating temperature, velocity, and density fluctuations, as well as modifying the plasma distribution function. Therefore any single instability that causes plasma inhomogenieties must be considered in terms of possible interactions with other, seemingly unrelated instabilities.

\subsubsection{Filamentation}

One laser-plasma instability that can impose strong inhomogenieties on the plasma is electromagnetic filamentation. A qualitative description of this instability begins with small fluctuations of the laser beam intensity in a perpendicular direction to the laser propagation. The regions of higher intensity will have a stronger pondermotive force, as given by Eqn. 2.2, and therefore these regions will expel plasma and have a lower density. These regions of plasma will then act as lenses (because of the different dielectric constants of the higher- and lower-density plasma), which will then focus more of the laser energy into these regions and increase the size of the fluctuations. The result of this effect is to break the beam up into small parallel "filaments" of higher intensity light and lower density plasma [14].

Recent results in 2D hydrodynamic codes [15] imply that steady-state behavior of the filaments may be rarely achieved in practice. In the simulations, filaments often take the form of "dancing beamlets" that deflect left and right (scattered by self-generated ion waves) and can break up into further sub-filaments. The result of this process is to impose both spatial and temporal incoherence between different regions of the laser field, 
limiting the length parameter over which instabilities can grow. However, this also leads to pockets of higher intensity fields which can cause higher-threshold laser-plasma instabilities to become important. Other effects of this filamentation process are a spatially varying temperature and even a modified electron distribution function throughout the plasma, as discussed in the previous section [13].

\subsubsection{Absorption Processes}

All of the above effects are underdense $\left(n<n_{c r}\right)$ phenomena that can couple energy from the laser to the plasma. More dramatic absorption occurs when a laser is incident upon an overdense plasma $\left(n>n_{c r}\right)$. The most straightforward coupling mechanism is collisional heating; electrons oscillate in the laser field (inverse bremsstrahlung absorption) and collide with the plasma ions, heating the plasma at the expense of the laser. This effect has been calculated analytically for both steep-gradient solid targets [16] as well as numerically for targets with a plasma density gradient, perhaps caused by a laser pulse longer than 1ps or even a lower-intensity laser pre-pulse [17].

However, all collisional coupling mechanisms are highly sensitive to the electronion collision frequency, $v_{\mathrm{ei}}$, which scales inversely to the number of particles in a Debye sphere. From Eqn. 1.1 it follows that $v_{\mathrm{ei}} \alpha \mathrm{T}_{\mathrm{e}}{ }^{-3 / 2}$. Therefore, as the intensity of the laser increases, raising the temperature of the plasma at the interaction region, collisional processes will sharply decrease. The effective collision frequency is further reduced when $\mathrm{v}_{\mathrm{Osc}}$ becomes comparable to the thermal velocity of the electrons [18] As a result, collisional absorption mechanisms decline in importance when $\mathrm{I}^{2}>10^{15} \mathrm{~W} \mathrm{~cm}^{-2} \mu \mathrm{m}^{2}$.

However, laser absorption continues to occur above these intensities; collisionless mechanisms can transfer energy to the plasma. Resonance absorption is one simple collisionless mechanism, where the electric field of the laser directly drives an electron plasma wave at the surface of critical density [19]. This excited plasma wave will 
eventually deposit its energy in the surrounding plasma, and can be responsible for up to $70 \%$ total absorption fractions, depending on the intensity and the polarization of the laser's electric field. This fraction tends to drop with increasing intensity, as will be discussed in the next section.

The details of resonance absorption can also change dramatically with the scale length of the plasma density gradient near the critical surface. At the critical density, the

electrons in an electron plasma wave oscillate with a spatial amplitude of $\sim v_{\text {osc }} / \omega_{p}$. Once this distance is larger than the scale length of the plasma gradient $\mathrm{L}$, then the resonance will break down. Instead, a "not-so resonant absorption" process can now be driven [20] where the electrons are directly heated by the laser field itself and thrown forward into the overdense plasma every laser cycle. From this model, the hot electron temperature created by this process can be calculated to scale as I $\lambda^{2}$ [21]. In the next section, we shall see that this scaling law does not continue to hold at intensities much higher than $\mathrm{I} \lambda^{2}=10^{16} \mathrm{~W} \mathrm{~cm}^{-2} \mu \mathrm{m}^{2}$.

\subsection{High Intensity Interactions (I $\left.\lambda 2>1017 \mathrm{~W} \mathrm{~cm}^{-2} \mu \mathrm{m}^{2}\right)$}

\subsubsection{Relativistic Effects}

At extremely high intensities, much of the physics of laser-matter interactions is determined by the large value of the dimensionless parameter $\alpha=v_{\text {osd }} / \mathrm{c}$. According to [2.1], this parameter can be greater than 1 when $I \lambda^{2}>10^{18} \mathrm{~W} \mathrm{~cm}^{-2} \mu \mathrm{m}^{2}$. Of course, the physical interpretation of $\mathrm{v}_{\mathrm{Osc}}$ as the peak velocity of an oscillating electron in the laser field is no longer correct; the actual velocity will never be larger than $\mathrm{c}$ due to the relativistic mass increase of the electron. The relativistically correct interpretation is $\alpha=\mathrm{p}_{\text {osc }} / \mathrm{mc}$ where $\mathrm{p}_{\text {osc }}$ is the peak momentum of the oscillating electron.

One immediate consequence of the relativistic electron motion is that the B-field of an intense laser is no longer negligible. For an electron oscillating in a laser field at a 
maximum velocity near the speed of light, the maximum Lorentz force is approximately the same as the maximum electric force:

$$
\left|\mathbf{F}_{\mathbf{B} \max }\right|=e|\mathbf{v} \times \mathbf{B}| \approx e c\left(\frac{E_{0}}{c}\right)=e E_{0}=\left|\mathbf{F}_{\mathbf{E} \max }\right|
$$

Here the maximum $B$-field of the laser $\mathrm{B}_{0}=\mathrm{E}_{0} / \mathrm{c}$. Note that the velocity of electrons (due to $E_{0}$ ) is in phase with the laser's B-field, but $\pi / 2$ out of phase with the laser's E-field. The Lorentz force on the oscillating electrons shifts from $+\mathbf{k}$ (the direction of laser propagation) to $-\mathbf{k}$ in half of a laser cycle. Therefore the B-field of the laser becomes an integral part of high-intensity laser-matter interactions.

Another direct consequence of relativistically oscillating electrons is that the electrons will relativistically gain mass by a factor $\gamma=\left(1-v^{2} / c^{2}\right)^{-1 / 2}=\left(1+\alpha^{2}\right)^{1 / 2}$. From Eqn. 1.2, this larger electron mass will lower the plasma frequency by a factor of $1 / \gamma$, which in turn raises the critical density and allows a given frequency laser to propagate into higher density plasmas.

\subsubsection{Collisionless Absorption}

While resonance (and "not-so-resonant") absorption are collisionless mechanisms, they can be limited by the $\mathbf{v} \times \mathbf{B}$ deflection of the electron orbits as described in Eqn. 2.8. This force can now excite electron plasma waves itself, and can potentially be a more efficient mechanism than resonance absorption at high intensities. [22,23]

One important factor in resonance absorption is that the incident laser has a Efield perpendicular to the $\mathbf{k}$-vector of the laser, while the critical density surface tends to lie in the $\mathbf{k}$ direction. Therefore resonance absorption is much stronger in p-polarized, oblique angle laser-solid interactions, where the E-field can point into the overdense plasma. 
This geometrical issue is less important for electron plasma waves driven by the $\mathbf{v}$ x B Lorentz force, which always points in the $\pm \mathbf{k}$ direction, and therefore tends to push electrons into the region of overdense plasma. As the laser field cuts off into the overdense plasma, it can form a strong accelerating gradient for single electrons. This is very analogous to a pondermotive potential, except now the driving mechanism is the forward Lorentz force rather than the radial electric force. If the energy of the electrons comes from the pondermotive potential of the laser, the temperature can be analytically calculated as [24]:

$$
T_{h o t}=\left[\left(1+I \lambda^{2} / 1.4 \times 10^{18}\right)^{1 / 2}-1\right] \times 511 \mathrm{keV}
$$

Additional calculations, simulations, and experiments up to $\mathrm{I} \lambda{ }^{2}=210^{18} \mathrm{~W} \mathrm{~cm}^{-2}$

$\mu \mathrm{m}^{2}$ concur that the escaping fast electrons have a temperature that scales as $\left(\mathrm{I} \lambda^{2}\right)^{1 / 3 / 1-2}$, less intensity-dependent than the simpler model presented in the previous section. [21,23]

Despite this agreement at lower intensities, it clearly would be instructive to perform electron temperature measurements into the regime $\mathrm{I}^{2}>10^{19} \mathrm{~W} \mathrm{~cm}^{-2} \mu \mathrm{m}^{2}$; this would provide a further test of the basic theory as well as provide insight to the feasibility of the fast ignitor fusion concept. Experiments at this intensity regime will be discussed in Chapters 4 and 5. 


\section{Chapter 3}

\section{Interactions Between Crossing Laser Beams}

\subsection{Background}

\subsubsection{Previous Work}

Energy transfer between two intersecting laser beams in a plasma directly addresses fundamental aspects of laser-plasma interactions and is also relevant to laserdriven inertial confinement fusion. Stokes coupling, as described in Section 2.1.2, is one mechanism by which energy can be transferred between beams; full mathematical details can be found in Appendix A. Eqn. [2.4] gives the frequency-matching and wavenumbermatching conditions that must be satisfied for resonant energy transfer, along with the condition that the driven plasma wave $\left(\omega_{\mathrm{s}}, \mathbf{k}_{\mathbf{s}}\right)$ be a normal mode of the plasma.

When the two lasers have a frequency difference near $\omega_{\mathrm{p}}$, the resonant Stokes wave can be a Langmuir wave according to the dispersion relation in Eqn. [1.8]. Driving a resonant wave in this matter has been accomplished [25], and further experiments have shown that the driven electron plasma wave can serve as an electron accelerator [26]. Energy transfer between the driving laser beams has also been demonstrated when the driven Stokes wave is a resonant electron plasma wave [27].

For laser beams of comparable frequency the difference will be small and Langmuir waves will never be resonant. Rather, low-frequency ion waves, with a dispersion relation given by Eqn [1.10], are more relevant for energy exchange between the lasers (and to a lesser extent the plasma). Resonant ion waves have been driven by 
microwaves [28] and also by two laser beams [29]. This latter experiment measured a modest transfer of energy mediated by a resonant ion wave, as evidenced by the fact that no energy transfer was observed for two laser beams of equal frequency.

Non-resonant ion waves have been produced with two identical frequency beams, which were found to have an effect on Stimulated Raman Scattering [30]. More recently, Lal et al. have observed energy transfer between two $\lambda=10.6 \mu \mathrm{m}$ wavelength laser beams [31], but this was during a transient period on the order of a few acoustic periods, during which energy transfer may occur between identical frequency beams [32,33].

These previous experiments were performed in subsonic plasmas. In a supersonic plasma $\left(\left|\mathbf{v}_{\mathbf{d}}\right| \geq \mathrm{c}_{\mathrm{S}}\right)$ the resonant ion wave can have zero frequency in the laboratory frame if $\omega_{i a}=0$ in Eqn. [1.10]. It has been proposed that for this condition, a ion wave could transfer energy between two identical frequency beams over many acoustic periods [3436]. This chapter will address new experiments that test this possibility.

\subsubsection{Relevance to Indirect Drive Fusion}

As noted in section 1.4.2, current designs for indirect drive fusion (such as NIF) involve the intersection of multiple laser beams of varying intensities as they enter a gasfilled hohlraum (see Fig. 1.1). Because the plasma flow leaving the hohlraum can be near supersonic [36], the ion wave frequency may be shifted to zero as described above. This in turn could allow a resonance that might transfer energy between the laser beams.

Figure 3.1 shows the geometry of a hohlraum experiment in the 10-beam Nova laser. Five equal-intensity beams are incident on each side of a cylindrical hohlraum. It is experiments in this geometry, more than any other, that have laid the scientific groundwork for the 192-beam NIF facility. Although there are crossing laser beams in the 10-beam geometry, a crossed-beam energy exchange is not likely because the resonant ion wave will be exactly perpendicular to the axis of the hohlraum. The plasma 


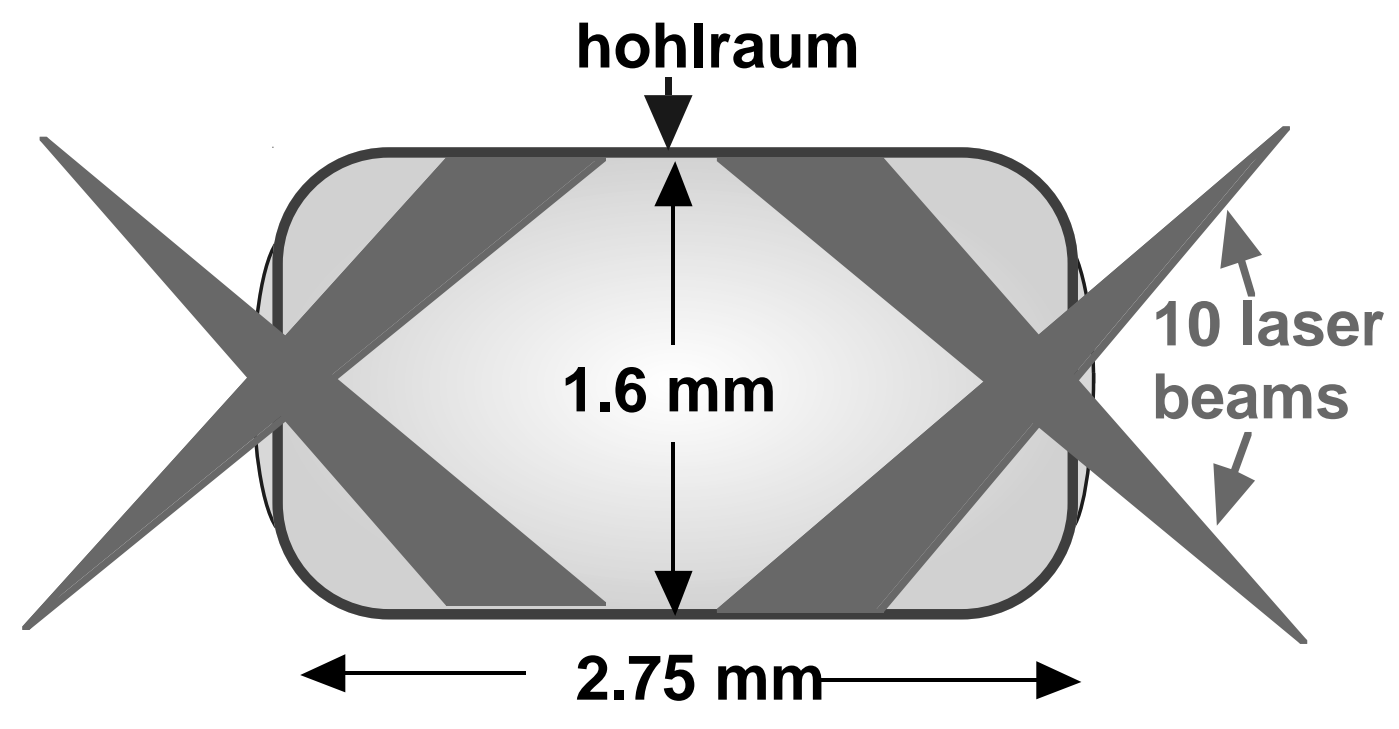

Figure 3.1. A schematic of the 10-beam geometry of NOVA indirect-drive experiments. All 10 beams are incident at the same angle with respect to the hohlraum axis.

flow out of the hohlraum, which will be directed along the hohlraum axis, will therefore not be in the correct direction to shift the ion frequency of the correct wave to zero. In other words, the $\mathbf{v}_{\mathbf{f}} \mathbf{k}^{\mathbf{k}}$ term from Eqn. 1.10 is zero in the 10-beam Nova geometry.

However, as seen in the comparison between Figures 1.1 and 3.1, the geometry of NIF will be substantially different from Nova. Four cones of laser beams will now cross in the Laser Entrance Hole (LEH) and beams in different cones will have a resonant ion wave with a component along the hohlraum axis. Hydrodynamic simulations using LASNEX [37] have shown that a sufficiently strong plasma flow in NIF might shift the frequency of these resonant ion waves to zero [38]. Therefore, there is a concern of energy transfer between beams in the NIF geometry.

This effect has been the subject of much theoretical work [33,39-41], and is a concern because energy transfer would have deleterious effects on the symmetry of the laser radiation inside the hohlraum. This in turn could limit or prevent the fusion gain from the fuel pellet, depending on the timing and severity of the asymmetry. ( $8 \% \mathrm{rms}$ 
balance is required among the 192 laser beams of NIF). Although the experiments presented below show that this "crossed-beam instability" is possible in principle, it does not directly address many of the conditions that will be found in NIF, and the relevance of this experiment to NIF will be discussed in Chapter 6.

\subsubsection{Relevance to Direct Drive Fusion}

Like indirect-drive fusion, direct-drive fusion schemes also required crossing laser beams. While there is no hohlraum in a direct-drive geometry, overlapping beams are required to raise the implosion symmetry balance to $\sim 1 \% \mathrm{rms}$ (as opposed to $8 \%$ for indirect-drive). This is accomplished both by smoothing techniques on individual beams (phase plates, 2-D SSD, polarization smoothing, etc.) as well as overlapping neighboring beams to smooth the overall beam profile.

In addition, the ablating plasma from a direct-drive fuel pellet can have significant flow velocity, although the very highest flows are found in low density plasmas far from the pellet. Together, one might expect the combination of flow and crossing-beams in direct drive to be even more serious than in indirect drive, as less energy transfer would be needed to destroy the implosion symmetry.

In the indirect-drive case, however, the laser beams come in at different cone angles, which leaves the resonant ion wave with a component along the hohlraum axis. There are not different cone angles in direct-drive geometries; all beams are pointed at the center of the fuel pellet. Therefore a flow that might shift the frequency of the resonant ion wave to zero would have to be a transverse flow, and not the radial flow that is typically present.

Although this might seem to eliminate crossed-beam effects as a concern in direct-drive fusion, a resonant interaction is still possible because of the finite f-number of the beams. Because each beam has a range of cone angles, energy at different cone 
angles can still interact, giving the resonant ion waves a radial component which can then be frequency-shifted by the radial flow. The size of this component will depend on two parameters: 1) the distance between the crossed-beam interaction and the center of the fuel pellet, and 2) the f-number of the incident laser beams. As the former parameter gets smaller and the latter parameter gets larger, the radial component of a potentially resonant ion wave increases.

A further concern is the 2-D SSD smoothing that is utilized on the Omega laser system and will likely be necessary for any direct-drive system. The spectral smoothing results in an increased bandwidth, which allows ion waves to be resonant even without a zero frequency: the frequency difference of the laser light is no longer always zero. This is also a concern in indirect-drive schemes (NIF), which may utilize SSD as well. While no crossed-beam experiments have been performed with SSD, four shots are scheduled in September 1998 on the Nova laser system to determine if the larger bandwidth associated with SSD might increase (or decrease) any crossed-beam interaction. While the results cannot be included in this thesis, they will be published in a special issue of Physics of Plasmas in May 1999.

\subsection{Experiment}

\subsubsection{Production and Diagnosis of the Flowing Plasma}

The work described here is the first measurement of steady-state energy transfer between identical frequency beams in a plasma with supersonic flow. The experiments were performed on the 10-Beam Nova laser facility at LLNL, using four $\mathrm{f} / 4.3$ beams with a wavelength $\lambda=351 \mathrm{~nm}$. Two of the beams (designated as "heater beams") were partially defocused to $800 \mu \mathrm{m}$ diameter spots, each spatially smoothed with a kinoform phase plate (KPP) [42] and containing $3 \mathrm{~kJ}$ of energy in a square pulse lasting from $\mathrm{t}=0$ to 
$\mathrm{t}=3 \mathrm{~ns}$. These two heater beams were incident $\left(40^{\circ}\right.$ to normal) on both sides of a $5 \mu \mathrm{m}$ thick Be $(Z=4)$ rectangular foil, 2 by $4 \mathrm{~mm}$ in size.

The exploding foil was initially modeled with LASNEX, using the heater beam parameters described above. A layer with Mach 1 flow $\left(\left|\mathbf{v}_{\mathbf{f}}\right|=\mathrm{c}_{\mathrm{S}}\right)$ was calculated to move out from the initial foil position over time, reaching a distance of $500 \mu \mathrm{m}$ from the foil at $\mathrm{t}=3 \mathrm{~ns}$. At this time the density along the center normal of the foil had reached a $1 \mathrm{~mm}-$ scale plateau of a roughly constant electron density $n_{e}=0.06 n_{c}$, where $n_{c}$ is the critical density for $351 \mathrm{~nm}$ light $\left(910^{21} \mathrm{~cm}^{-3}\right)$.

Not to overly rely on the LASNEX prediction, the flow velocity was then experimentally characterized with a Thomson scattering technique [43]. A lower intensity $\lambda=526 \mathrm{~nm}$ probe beam was focused in a $100 \mu \mathrm{m}$ FWHM spot, $500 \mu \mathrm{m}$ from the center foil position. The incident probe beam angle was chosen such that the k-vector of the probe $\mathbf{k}_{0}$ minus the $\mathrm{k}$-vector of the scattered light $\mathbf{k}_{1}$ (in the direction of the detector), matched to the k-vector $\mathbf{k}_{\mathrm{s}}$ of a plasma wave of interest (according to Eqn. 2.4). This matching plasma wave (arising from the thermal background) was an ion acoustic wave that lay within $15^{\circ}$ of the foil normal, and therefore within $15^{\circ}$ of the flow velocity vector vf. This wave, according to Eqn [1.10], has a term $\mathbf{v}_{\mathbf{f}} \mathbf{k}_{\mathrm{s}}$ in its frequency, and therefore the frequency shift of the Thomson-scattered light is a diagnostic for the velocity of the plasma. More specifically, when the scattered frequency $\omega_{1}$ is equal to the initial probe frequency $\omega_{0}$, Eqn. [2.4] implies that the laboratory-frame frequency of the plasma wave $\omega_{\mathrm{S}}$ is zero. This in turn implies (by Eqn [1.10]) that a Mach 1 plasma flow exists in the $\mathbf{k}_{\mathrm{s}}$ direction, opposite to the propagation of the diagnosed plasma wave. It is precisely the ion waves of this type $\left(\omega_{\mathrm{S}}=\omega_{\mathrm{ia}}=0\right)$ which can act as energy-transferring mediators between two crossing laser beams. 


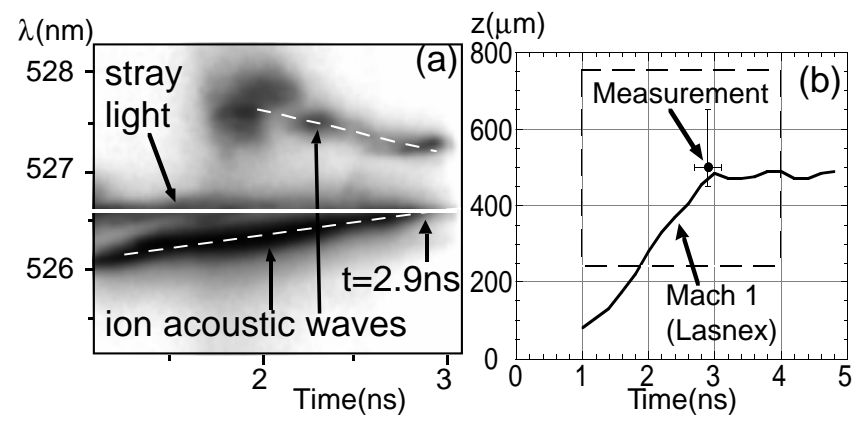

Figure 3.2. (a) Thomson scattering image from a $\lambda=526.6 \mathrm{~nm}$ probe focused at $\mathrm{z}=500 \mu \mathrm{m}$ from the original foil position, resolved in wavelength and time. The upshifted ion acoustic wave feature overlaps the unshifted light at $\mathrm{t}=2.9 \mathrm{~ns}$, signifying a Mach 1 plasma flow. (b) The LASNEX calculation of the Mach 1 flow location is plotted against time. The measurement from Fig. 1a is shown in comparison. The dashed box represents the typical spatial and temporal extent of the crossed beams in the primary experiment.

The frequency- and time-resolved Thomson scattered light is shown in Figure 3.2a. At $t=2.9 n s$ the frequency up-shifted ion wave feature overlaps the stray light, signifying that $\omega_{\mathrm{ia}}=0$ and that a Mach 1 plasma flow was present at an angle of $15^{\circ}$ to the target normal. The correction factor for the plasma flow velocity normal to the foil is $\left(\cos 15^{\circ}\right)^{-1}=1.035$, which is negligible compared to the error arising from the spectral line width of the measurement. Figure 3.2b shows that this measured Mach 1 position $(\mathrm{z}=500 \mu \mathrm{m}, \mathrm{t}=2.9 \mathrm{~ns})$ is consistent with the LASNEX calculation.

Further information concerning plasma temperature can be extracted from the separation between the two ion wave features. As described in Section 2.1.3, the two ion wave features are separated by $2 \omega_{\mathrm{ia}}$, and therefore this data serves as a time history of the ion acoustic frequency. The temperature of the plasma can be determined using Eqn [1.9], with one large complication. For low $\mathrm{Z}$ materials, the $\mathrm{T}_{\mathrm{i}}$ term cannot be ignored, and therefore there are two unknowns: $T_{e}$ and $T_{i}$. In order to determine $T_{e}$, an assumption must be made concerning the $\mathrm{T}_{\mathrm{e}} / \mathrm{T}_{\mathrm{i}}$ ratio; this is where the majority of the 
error enters into the temperature measurement. LASNEX predicts a $T_{e} / T_{i}$ ratio of about 2, and this was assumed to be correct to within $25 \%$.

With this assumption, the measured electron plasma temperature at $t=2.9 \mathrm{~ns}$ is $0.8 \pm 0.2 \mathrm{keV}$, lower than the predicted $1.2 \mathrm{keV}$ at this location. This could mean that the correspondence of the Mach 1 position may be somewhat coincidental, as the flow velocity which corresponds to Mach 1 is dependent on temperature. The error bars of the Mach 1 measurement are largest in the direction away from the foil due to the possibility that the beam was deflected by the plasma density gradient, with a maximum error defined by the spatial view of our diagnostic. Even with this effect, the error in the Mach 1 measurement is much smaller than the spatial and temporal extent of the region sampled in the main experiment.

\subsubsection{Crossing Beams in the Flowing Plasma}

The main experiment was then performed by crossing two additional $\lambda=351 \mathrm{~nm}$ beams in the exploding foil plasma. We refer to the higher-intensity beam as the "pump", and the lower-intensity beam as the "probe". As shown in figure 3.3, these beams arrived from opposite directions, separated by an angle of $152^{\circ}$. Both the pump and probe were incident at $14^{\circ}$ from the normal of the foil (the z-axis, defined in figure 3.3 ), and the resultant ion wave was therefore aligned to the plasma flow along the $\mathrm{z}$-axis. The pump and probe beams were originally focused at a location $\mathrm{z}=-500 \mu \mathrm{m}$ from the $\mathrm{z}=0$ initial foil position. The pump has a higher frequency in the frame of the flowing plasma on the $-\mathrm{z}$

half of the foil, and therefore the resonance would be expected to transfer energy from the pump to the probe and the ion wave.

The probe light transmitted through the plasma was incident on a frosted fusedsilica plate $1.5 \mathrm{~m}$ from the target, and the scattered light was then imaged onto a fast 


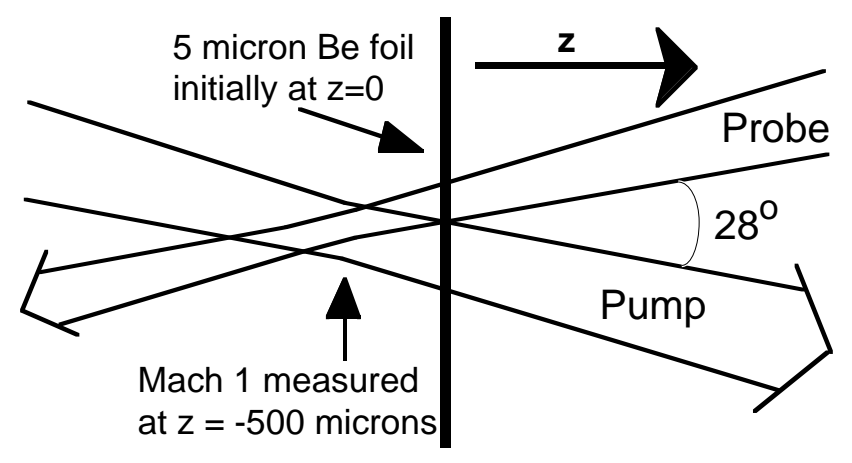

Figure 3.3. The experimental geometry is shown. The $5 \mu \mathrm{m}$ Be foil is initially at $\mathrm{z}=0$, where $\mathrm{z}$ is the normal to the foil in the direction of the Pump beam propagation. At $\mathrm{t}=0 \mathrm{~ns}$ two heater beams (not shown) illuminate the foil from both sides. At $t=1$ ns the Pump and Probe laser beams intersect at a known distance from the foil, at a $152^{\circ}$ angle. The diamond-shaped crossing region can have a z-extent of $850 \mu \mathrm{m}$ to $1300 \mu \mathrm{m}$ depending on the focal spot sizes, but $>75 \%$ of the intensity intersects in a region only half this size.

photodiode [44]. Post-processing of the photodiode signal helped correct for the finitetime response of both the large scatter plate and the diode. The final absolute uncertainty in the transmission measurements is $\pm 14 \%$ ( $\pm 20 \%$ for time scales <100ps), and the relative uncertainty between different shots is $\pm 10 \%$.

The specifications of the crossing beams were as follows: the pump beam was identical to the heater beams (square pulse, $3 \mathrm{~kJ}$ in $3 \mathrm{~ns}$, KPP), but arrived $1 \mathrm{~ns}$ late, staying on from $\mathrm{t}=1 \mathrm{~ns}$ to $\mathrm{t}=4 \mathrm{~ns}$. The pump was focused to a $340 \mu \mathrm{m}$ full-diameter spot, reaching an intensity of $10^{15} \mathrm{Wcm}^{-2}$.

The probe beam had a typical energy of $0.2 \mathrm{~kJ}$, and two focal spots were used. For the first part of the experiment, no phase plate was used on the probe, allowing a focused FWHM of $100 \mu \mathrm{m}(170 \mu \mathrm{m}$ full diameter). The probe's pulse shape was a $3 \mathrm{~ns}$ upward ramp, beginning at $\mathrm{t}=1 \mathrm{~ns}$ and reaching a peak of $150 \mathrm{GW}$ at $\mathrm{t}=4 \mathrm{~ns}$. Then, in order to change the intensity ratio between the crossing beams, a KPP was added to the probe 
beam for the second part of the experiment. This increased the spot size to $340 \mu \mathrm{m}$ (full diameter between Airy minima). Also, the probe's pulse shape was changed to a $4 \mathrm{~ns}$ square pulse, extending from $t=1 n s$ to $t=5 n s$. The increased size of the probe stretched the z-extent of the diamond-shaped region where the full beams intersected from $850 \mu \mathrm{m}$ to $1350 \mu \mathrm{m}$. The length of the region where $>80 \%$ of the energy intersected increased from $500 \mu \mathrm{m}$ to $800 \mu \mathrm{m}$.

\subsubsection{Results}

The transmission of the first (smaller spot size) probe beam is plotted against time in figure 3.4a. With no pump beam present, the transmission of the probe through the exploding foil stabilized at 50-60\%. With the addition of the pump beam, crossing the probe at a location $\mathrm{z}=-500 \mu \mathrm{m}$, the transmission of the probe increased to near $100 \%$ on short timescales. However, when the two beams were crossed at a location $\mathrm{z}=-750 \mu \mathrm{m}$, the probe transmission returned to the previous $50-60 \%$ level. Because the probe passed through the bulk of the exploding foil plasma before reaching the crossing region, this region had an average intensity ratio $\mathrm{I}_{\text {pump }} / \mathrm{I}_{\text {probe }}$ of $\sim 3$.

By adding a KPP to the probe beam as described above, the ratio $\mathrm{I}_{\text {pump }} / \mathrm{I}_{\text {probe }}$ was increased to $\sim 25$ for the second part of the experiment. Figure $3.4 \mathrm{~b}$ shows the transmission of this lower-intensity probe beam, both with and without the pump beam. The no-pump transmission was nearly identical to the previous case despite the different pulse shapes, evidence that the low-intensity probe beam was not strongly affecting the plasma. With the pump beam present, the transmission was again increased to $\sim 100 \%$ levels when the beams were crossed at $\mathrm{z}=-500 \mu \mathrm{m} ; \sim 80 \%$ levels when the beams were crossed at the original foil position $(\mathrm{z}=0)$; and no significant transmission enhancement when the beams were crossed at $\mathrm{z}=+500 \mu \mathrm{m}$. 

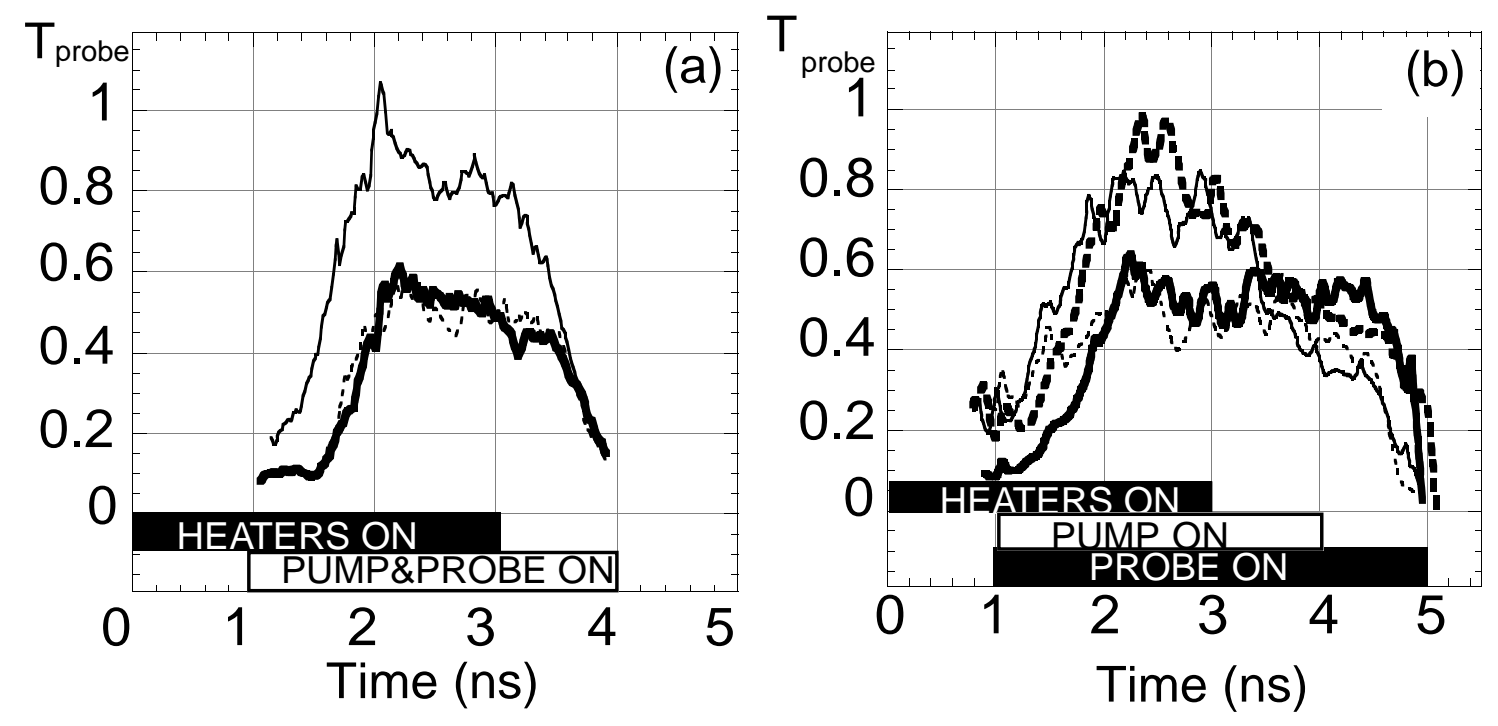

Figure 3.4. (a) Probe transmission fraction $\left(\mathrm{T}_{\text {probe }}\right)$ is plotted against time for a Pump/Probe intensity ratio of $\sim 3$. The thick solid line is the probe-only (no pump) condition. The other data show beam-crossing locations of $z=-500 \mu \mathrm{m}$ (thin solid line) and $\mathrm{z}=-750 \mu \mathrm{m}$ (thin dashed line). (b) Probe transmission fraction is plotted against time for a Pump/Probe intensity ratio of $\sim 25$. The thick solid line is the probe-only condition. The other data show beam-crossing locations of $\mathrm{z}=-500 \mu \mathrm{m}$ (thick dashed line), $\mathrm{z}=0 \mu \mathrm{m}$ (thin solid line), and $\mathrm{z}=+500 \mu \mathrm{m}$ (thin dashed line).

For each transmission measurement, a linear gain factor can be computed by simply dividing the crossed-beam transmission by the no-pump transmission. Although the early-time peak gains are large $(>3)$ in both cases where the beams were crossed at $\mathrm{z}=-500 \mu \mathrm{m}$, the corresponding errors are large as well because of the lower no-pump transmission values at these times. A more quantitative gain measurement can be made by averaging the gain over $2 \mathrm{~ns}<\mathrm{t}<3 \mathrm{~ns}$, the time period when the Mach 1 flow velocity is calculated to be between $\mathrm{z}=-300 \mu \mathrm{m}$ and $\mathrm{z}=-500 \mu \mathrm{m}$. These averages are plotted against 


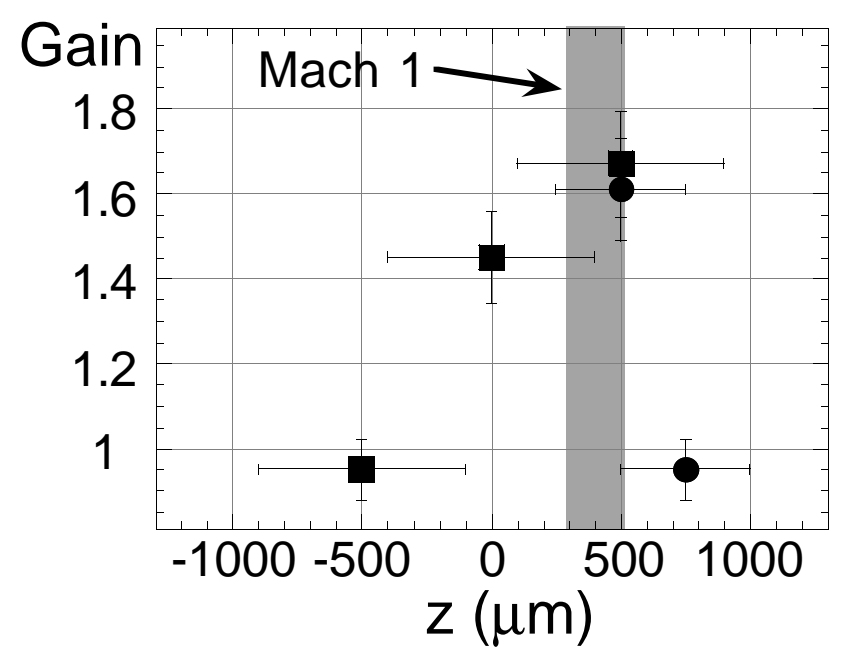

Figure. 3.5. The gain factor of the probe beam (averaged from $2 \mathrm{~ns}<\mathrm{t}<3 \mathrm{~ns}$ ) is plotted versus position for each crossed-beam location . Circles are for Pump/Probe intensity ratios of 3; squares are ratios of 25. The large horizontal error bars represent the region over which $>80 \%$ of the crossed beams overlap. The shaded region represents the calculated Mach 1 location during the $2 \mathrm{~ns}<\mathrm{t}<3 \mathrm{~ns}$ period.

position in Figure 3.5. The large horizontal error bars represent the extent in the z-axis of the high-intensity diamond-shaped crossing region of the two beams. The maximum gain values of $\sim 1.6$ occurred when the crossing region overlapped the Mach 1 region; little gain was observed when the beams were crossed outside this region. This dependence on position is strong evidence of a resonant process.

\subsection{Analysis}

\subsubsection{Possibility of Pump-Heating}

Increased transmission of the probe beam in the presence of a pump is not by itself absolute evidence of energy transfer; alternatively, the pump might heat the plasma and thereby decrease the inverse bremsstrahlung absorption of the probe beam. 
LASNEX simulations show no pump beam effect on the plasma density or Mach 1 location, but do show a higher temperature plasma when the pump is on. This temperature change increases the peak theoretical probe transmission from $60 \%$ to $70 \%$, but cannot explain the observed $\sim 100 \%$ transmission.

In addition, this effect is calculated to occur late in the pump pulse, from $t=3 \mathrm{~ns}$ onward, rather than the earlier $\mathrm{t}=\sim 2 \mathrm{~ns}$ where our peak transmission is observed. Further evidence against this pump-heating scenario includes a measurement of the transmission of the pump beam; this is compared with the probe beam transmission in Fig 3.6. The pump beam transmission peaks later in time $(\mathrm{t}=3.2 \mathrm{~ns})$, at a level of $55 \%$, while the amplified probe signal peaks earlier $(\mathrm{t}=2.4 \mathrm{~ns})$ and with much higher transmission levels.

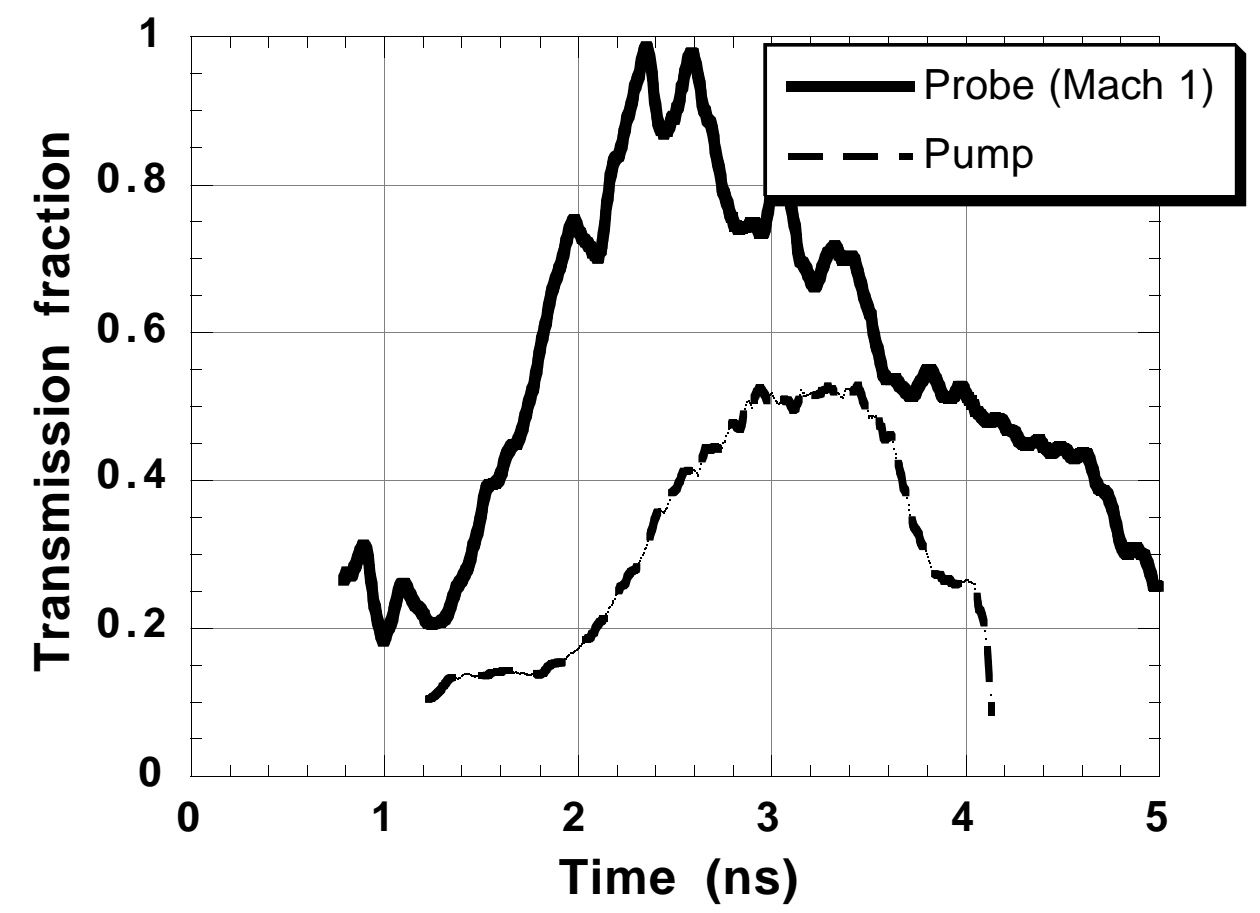

Figure 3.6. The transmission fraction of the pump beam and probe beam are plotted against time. The probe transmission shown here was a shot in which significant enhancement was seen, corresponding to the thick dotted line in Figure 3.4b. 
Because the two beams are not exactly co-linear, any pump-induced heating should have primarily increased the pump transmission.

Finally, all enhanced transmission is seen to end at $\mathrm{t}=4 \mathrm{~ns}$ when the pump beam turns off, as expected from a crossed-beam effect. Any pump-heating effect would not turn off with the pump beam, as the hot plasma would continue to allow a greater transmission for times $>4 \mathrm{~ns}$.

To summarize this point, the timing and strength of the observed effect is inconsistent with a pump-heating scenario but consistent with resonant energy transfer between crossed beams. The positional scaling from Figure 3.5 is the strongest argument in favor of a resonant process.

\subsubsection{Scaling of Energy Transfer}

Given that the experimental results seem to point to resonant energy transfer, it is important to analyze the absolute levels and scaling of this effect. While the five data points in Figure 3.5 offer a limited data set from which to extrapolate, some interesting observations can still be made.

Figure 3.5 shows that the measured average gain at $\mathrm{z}=-500 \mu \mathrm{m}$ is roughly the same $(\sim 1.6)$ for both experimental intensity ratios. If the resonant ion wave were saturated (see section A.4 in the Appendix) one would expect the gain to increase with increasing $\mathrm{I}_{\text {pump }} / \mathrm{I}_{\text {probe }}$. In an unsaturated case, however, Figure A.1 predicts little difference between the energy transfer percentages for different intensity ratios. Our equal gain measurements, however, suggest that this process is not in a saturated regime. This conclusion concurs with previous resonant energy-transfer scaling [29] but is at odds with the near-complete transfer of the pump energy predicted by assuming a linearly driven ion wave in a homogenous plasma (Eqn. [2.6]). 
As described in Chapter 2, there are many possible mechanisms that could limit the amount of energy transferred, such as non-Maxwellian distribution functions driven by filamentation of the pump beam. Many of these mechanisms have been employed in the past to explain low amounts of energy transfer [29]. The pump beam in this experiment is in an intensity range where filamentation occurs, and therefore we expect somewhat modified plasma distribution functions in this experiment. While a quantitative estimate of gain reduction by this mechanism is difficult to estimate, the gain length L over which the instability may occur can be shortened dramatically, splitting up the "resonant" volume into small, disconnected regions.

Numerical simulations of plasmas with strong gradients show further reductions of the amplification. BZOHAR, a two-dimensional electromagnetic code that uses particle ions and Boltzmann fluid electrons [45], has been used to perform simulations of this experiment on small spatial scales [46]. These simulations suggest that the resonant ion waves and the probe amplification saturate after several ion acoustic periods, but the energy gain quickly relaxes (after 40ps) and becomes nearly proportional to the input probe intensity, as seen in the experiments. BZOHAR arrives at this "linear" condition by means of nonlinear detuning and nonlinear localization of the ion wave resonance.

An additional comment should be made concerning the single "Mach -1 " data point, where the beams were crossed on the wrong side of the exploding foil (at $\mathrm{z}=500 \mu \mathrm{m})$. Because the intensity of the beams was not changed (except due to differing levels of attenuation by the plasma) we still refer to $I_{\text {pump }}$ as the high-intensity beam and $I_{\text {probe }}$ as the low-intensity beam. However, in the frame of the plasma $I_{\text {pump }}$ is no longer the higher-intensity wave. In the notation of Appendix A, where $\mathrm{I}_{2}$ is the intensity of the lower frequency crossed-beam and $\mathrm{I}_{3}$ is the intensity of the higher-frequency beam, $\mathrm{I}_{\mathrm{pump}}$

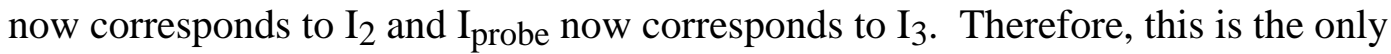
experimental point for which $\mathrm{I}_{2}>>\mathrm{I}_{3}$. 
Because the energy in crossed-beam interactions flows from the highestfrequency wave to the lower-frequency waves, in the "Mach -1" interaction one would expect energy to flow from the probe to the pump, rather than the reverse (see section A.2). If this in fact occurred the experiment would have measured a drop in probe transmission, giving the Mach -1 data point a "gain" parameter less than unity. But although the intensity product $\mathrm{I}_{2} \mathrm{I}_{3}$ was the same as in the Mach 1 case, and one might expect similar-amplitude ion waves to be driven, Eqn. [2.6] clearly shows that the energy transfer is driven by the intensity of the high-frequency wave $\left(\mathrm{vosc}_{\mathrm{OSc}}^{2}\right)$. Therefore, the "Mach -1" data point represents a situation where the low-intensity probe is a weak driving wave, too weak to drive the three-wave instability that was driven by the pump beam at Mach 1 .

In summary, enhanced transmission of a laser beam is observed when it is crossed with a higher-intensity beam of the same frequency in a flowing plasma. Positional and temporal scaling of this effect demonstrates that this is due to a resonance with an ion wave that has zero frequency in the laboratory frame, making this the first observation of steady-state energy transfer between identical frequency laser beams. The observed intensity gain of $\sim 1.6$ in two different intensity regimes suggests that the resonant energy transfer is responding linearly to the driving laser beams. Further analysis of this experiment and its relevance to laser fusion schemes can be found in Chapter 6. 


\section{Chapter 4}

\section{Measurements of Hot Electrons Produced by High Intensity Laser-Solid Interactions}

\subsection{Background Physics}

\subsubsection{Previous Work}

In the field of laser-matter interactions, hot electrons have been a concern for many decades. In underdense $\left(n<n_{c r}\right)$ plasmas, hot electrons are produced by the damping of laser-produced electron plasma waves. Until recently, most laser-driven fusion schemes viewed these laser-heated electrons as a nuisance which could pre-heat the fuel pellet and prevent a uniform compression. Laser-induced acceleration of electrons was first viewed in a positive light by the particle acceleration community [25], and today there is much research into high-gradient electron accelerators using laserplasma interactions.

But these applications use underdense plasmas in order to acheive the highest electron energies, and consequently the total number of accelerated electrons is limited by the plasma density, among other considerations. Fast ignitor fusion, on the other hand, requires electron beams with kilojoules of total energy but modest single-electron energies $(\sim 1 \mathrm{MeV})$. The high densities of the fusion target and the necessary large number of electrons require laser-plasma interactions at the critical density, with the hot electrons then propagating into a solid target. 
Previous experiments have measured these hot electrons at lower intensities than are now available [47-53]. One recent experiment using strongly relativistic intensities $\left(\mathrm{I} \lambda^{2}>10^{19} \mathrm{~W} \mathrm{~cm}^{-2} \mu \mathrm{m}^{2}\right)$ measured high energy electrons in vacuum after leaving a foil target in which they were produced [54]. This technique, while able to accurately measure the spectrum of the highest-energy electrons, has several main drawbacks. One is that the solid angle of collected electrons is typically small, and not representative of the total number of electrons created in the interaction. The second drawback is that the electrons are measured after they leave the target, and their energy can be modified by the electrostatic potential of the remaining positively-charged target. This will not greatly affect the energies of the fastest electrons, but the bulk of the electron distribution may be misrepresented by these direct measurements. Finally, very large currents may not propagate in vacuum, as explained in section 4.1 .3 below; only in-target measurements can hope to measure anything greater than the vacuum current limit.

Many other experiments [47-51,53] have utilized $K_{\alpha}$ x-ray emission from buried layers in multilayer targets to diagnose the electrons. This is an indirect method for measuring the electrons before they leave the target via the inner shell ionization of a tracer material at a known depth in the target. By varying the depth of the tracer layer, an electron spectrum can be inferred from the corresponding change in the $K_{\alpha}$ X-ray yield. This method allows a full integration over all electrons with enough energy to propagate to the buried layer, but it does not provide the spectral resolution that is available from direct electron detection techniques.

For the purposes of experimentally addressing the feasibility of the Fast Ignitor technique presented in Chapter 1 , the $K_{\alpha}$ technique is the most appropriate. It allows the best measurement of the total conversion efficiency from laser energy into fast electrons, and accomplishes this in-target. While the details of the electron energy spectrum might have minor consequences for a fast ignitor scheme, the primary parameter is simply the 
mean energy of the electrons; this will determine where the most energy will be deposited in a DT fuel pellet. For these reasons, this chapter will experimentally address laserproduced hot electrons via the $K_{\alpha}$ technique.

\subsubsection{Single Electron Energy Deposition}

The $K_{\alpha}$ technique is an indirect one; information concerning the laser-produced electrons must be inferred from the strength of the x-ray signal. For this reason, it is important to understand the behavior of electrons as they propagate through the target.

Fortunately, the behavior of a fast electron in cold matter is well understood, and it can be modeled to a high degree of accuracy. Additional complications emerge when large numbers of electrons heat the material, create magnetic fields, and build up large space-charges; these complications will be addressed in the next section.

From a single-electron standpoint, an electron will undergo bremsstrahlung scattering off of the stationary atoms, decelerating the electron and radiating away some of its energy as x-rays. In addition, electrons can inelastically collide with the electrons of the stationary atoms, either raising them to an excited state or removing them from the atom altogether. More x-rays are released as these excited or ionized atoms relax to their ground state.

The linear rate at which electron energy is deposited is given by the Bethe-Bloch formula [55]:

$$
\frac{\partial E_{e}}{\partial s}=\frac{-2 \pi e^{4} Z n_{A}}{E_{e}} \ln \left(\frac{2 E_{e}}{\langle I P\rangle}\right)
$$

Here $\mathrm{E}_{\mathrm{e}}$ is the energy of the electron, $\mathrm{n}_{\mathrm{A}}$ is the atomic density, and $\langle\mathrm{IP}\rangle$ is the average ionization potential of the material. This is an approximation that assumes the atoms in the material have a near-equal number of protons and neutrons, good for moderate- $Z$ materials except hydrogen. This equation can be integrated to find the range 
of an electron of given energy, but this range is measured along the path of the electron. Because of the multiple inelastic scattering events that the electron encounters, it is more difficult to analytically determine the linear distance that an electron will propagate into a given material. The situation is further complicated by an energy dependence of the typical scattering angle of the electron. While extensive electron propagation tables are available, the most accurate way to model this process is through a Monte-Carlo computer code, which can randomize the collisions and determine the distributions of probable trajectories.

When an electron (or photon) collides with an inner K-shell electron and removes it from the atom, there is a probability that an L-shell electron will radiatively decay into the K-shell vacancy. This decay produces a $K_{\alpha}$ X-ray with a known energy, depending on the type of atom and its charge state. Alternate processes by which the K-shell vacancy may be filled include $K_{B}$ radiation (radiative decay of an M-shell electron into the K-shell vacancy) and an Auger process, by which an electron from the L-shell decays into the K-shell vacancy but the energy is taken off by another electron.

Because a K-shell vacancy can be created by both electrons and photons of sufficient energy, careful analysis must be made of the photo-pumped $K_{\alpha}$ x-rays. Unlike electrons, photons of sufficient energy are preferentially absorbed by the inner shell electrons, so a photon has a larger K-shell cross section than an electron of comparable energy. Under certain circumstances, the secondary x-rays produced by an electron (via bremsstrhalung and other processes) can be nearly as large a source of $K_{\alpha}$ x-rays as the electron itself. Therefore a comprehensive account of the $K_{\alpha}$ x-rays produced by electrons must include these secondary effects.

To this end, the Monte-Carlo electron-transport code ITS [56] was used to interpret the data. This code is widely used in the electron acceleration community, and has been tested and benchmarked against a wide array of experiments. The output of this 
code was the number of $K_{\alpha} \mathrm{x}$-rays per steradian emitted from a given target in a particular direction, normalized to the number of source electrons. In addition to calculating the electron transport and ionization, ITS also computed the x-ray continuum produced by bremsstrahlung of the fast electrons and the resulting photo-ionization of the target. Further discussion of the ITS code will continue below.

\subsubsection{Electron Beams in Matter}

Monte-Carlo modeling codes do not take collective effects into account in a selfconsistent manner. Because of this, large fields produced by an intense beam of electrons must be analyzed with different techniques. As high currents are expected from even a modest conversion of laser energy into electrons, these effects can be strong enough to dominate the single-electron effects discussed in the previous section.

Just as local charges are shielded by a plasma (over a spatial scale of $\lambda_{D}$ ), a plasma will also attempt to expel magnetic fields by shielding local currents as well. The mechanism is that a changing magnetic field induces an electric field which produces a current to exactly cancel the new magnetic field. Therefore a beam entering a plasma will lead to the generation of a "return current" that will tend to neutralize the magnetic field of the original beam. This process, like Deybe shielding, has a gradient; due to finite electron inertia, there is a skin depth over which magnetic fields are shielded:

$$
\lambda_{m}=c / \omega_{p e}
$$

Because of this imperfect shielding, a beam in the plasma will never be perfectly canceled by the return current. Although the net current may be much smaller than the original beam, some level of magnetic field will be produced inside the beam itself. This field will change the trajectories of the very electrons that make up the current, and a strong enough field will prevent a beam from propagating at all. This will begin to occur 
when the outer-most electrons in the beam (in the presence of the strongest B-field) execute $\pi / 2$ orbits that fully negate their original transverse velocity. For a uniform, cylindrical beam of electrons with energy E this can be computed to occur at an "Alfven current":

$$
I_{A}=\frac{c \sqrt{E^{2}+2 m c^{2} E}}{e}
$$

Because of the cgs units, $\mathrm{I}_{\mathrm{A}}$ is in the unfamiliar units of statamperes; it must be divided by $310^{9}$ to yield amps. It is not a forgone conclusion, however, that no beam can propagate through a plasma if $\mathrm{I}>\mathrm{I}_{\mathrm{A}}$. A hollow-ring current distribution, for example, can allow larger currents than a uniform distribution. Other geometries might be possible if one allows for curving electron orbits that wind their way through the plasma. It is unclear how large a current can exist in a plasma, but unshielded currents an order of magnitude greater than the Alfven current are unlikely to be physically possible.

For the fast ignitor scheme, a single Alfven current would not be nearly sufficient to spark a fusion reaction; currents of $10^{4} \mathrm{I}_{\mathrm{A}}$ would be more relevant. For such a current, a local return current of at least $0.99910^{4} \mathrm{I}_{\mathrm{A}}$ would be necessary to lower the magnetic fields to a level that might still allow the propagation of the original beam. This is a high degree of current cancellation, and it is important to determine if the magnetic skin depth from Eqn. 4.2 might be consistent with such a return current.

The simplest model, as in the derivation of the Alfven current, is to assume a cylindrical beam of uniform density. If the current in the beam is $\mathrm{I}_{\mathrm{b}}$ and the radius of the beam is $\sigma_{\mathrm{b}}$, an equal return current $\left(-\mathrm{I}_{\mathrm{b}}\right)$ will be drawn in an opposing beam of radius $\left(\sigma_{\mathrm{b}}\right.$ $\left.+\lambda_{\mathrm{m}}\right)$. This wider beam is due to the magnetic skin depth; the B-field will be completely shielded within a distance of $\lambda_{m}$. Locally, because of the different beam sizes, the return current inside the original beam area will be less than $\mathrm{I}_{\mathrm{b}}$ by a geometrical factor of $\left(\sigma_{b} / \sigma_{b}+\lambda_{m}\right)^{2}$. Therefore it is the net current in this area that will be constrained by the 
Alfven limit:

$$
I_{b}\left(1-\frac{\sigma_{b}^{2}}{\left(\sigma_{b}+\lambda_{m}\right)^{2}}\right) \equiv \frac{I_{b}}{N_{A}} \leq I_{A}
$$

Here $\mathrm{N}_{\mathrm{A}}$ has been defined as the number of Alfven currents which is possible to propagate in a directional beam, when taking into account the magnetic shielding from the equal return current. Rewriting Eqn. 4.2 in terms of the laser wavelength $\left(\lambda_{0}\right)$ and the plasma density, $\lambda_{m}=\left(\lambda_{0} / 2 \pi\right)\left(n_{c r} / n\right)^{1 / 2}$. If $\sigma_{b}$ is assumed to be the radius of the laser spot as well as the electron beam (and $\sigma_{\mathrm{b}} \gg \lambda_{\mathrm{m}}$ ), $\mathrm{N}_{\mathrm{A}}$ can be rewritten as:

$$
N_{A}=\pi \frac{\sigma_{b}}{\lambda_{0}}\left(\frac{n}{n_{c r}}\right)^{1 / 2}+1
$$

This is a simple result that seems to have been overlooked in the fast ignition literature and is derived here for possibly the first time. If the laser spot size radius is about 7 laser wavelengths, Eqn. 4.5 shows that a one-directional current of 23 Alfven currents is the limit in a critical density plasma. Arbitrarily large currents are possible in plasmas of sufficient density. In a fast ignitor application the peak density would be even greater, allowing still larger currents. It should again be noted that these formulas only apply for idealized uniform cylindrical beams, and a more realistic beam profile would result in somewhat different limits on the maximum current in a plasma. Also, any increase of the magnetic skin depth, either due to the "anomalous skin effect" or lower hybrid turbulence will decrease $\mathrm{N}_{\mathrm{A}}$. Finally, the Alfven limit can be relaxed by the addition of electrostatic forces, which could counter the large Lorentz force on the beam electrons. Further complications are also not taken into account in this simple model. Comparison of this model with the experimental results will be discussed in Chapter 6.

An additional way in which multi-Alfven currents could propagate in a soliddensity plasma is in the form of a large number of small filaments, each on the scale of the magnetic skin depth. This possibility will also be discussed in Chapter 6 . 


\subsection{Methods}

\subsubsection{Description of 100TW Laser}

The experiments presented here were performed at the Nova laser facility at LLNL, on a beamline known as the 100Terawatt laser. [57] This laser is a mixedamplifier system (see Figure 4.1), with a high-bandwidth front end (Ti-doped Sapphire amplifiers) that produces $50 \mathrm{~mJ}$ of $1.06 \mu \mathrm{m}$ wavelength pulses at $10 \mathrm{~Hz}$. These pulses are compressible to $320 \mathrm{fs}$ due to their large $8.0 \mathrm{~nm}$ bandwidth, but are generally chirped (stretched) to a long Gaussian pulse with a FWHM of 1.5ns. One of these pulses is selected to propagate into the second part of the system, a series of Nd-doped glass amplifiers. These amplifiers have a smaller bandwidth, but allow for higher peak intensities because of the longer lifetime of the population inversion. Some bandwidth can be restored by alternating between phosphate glass amplifiers and silicate glass

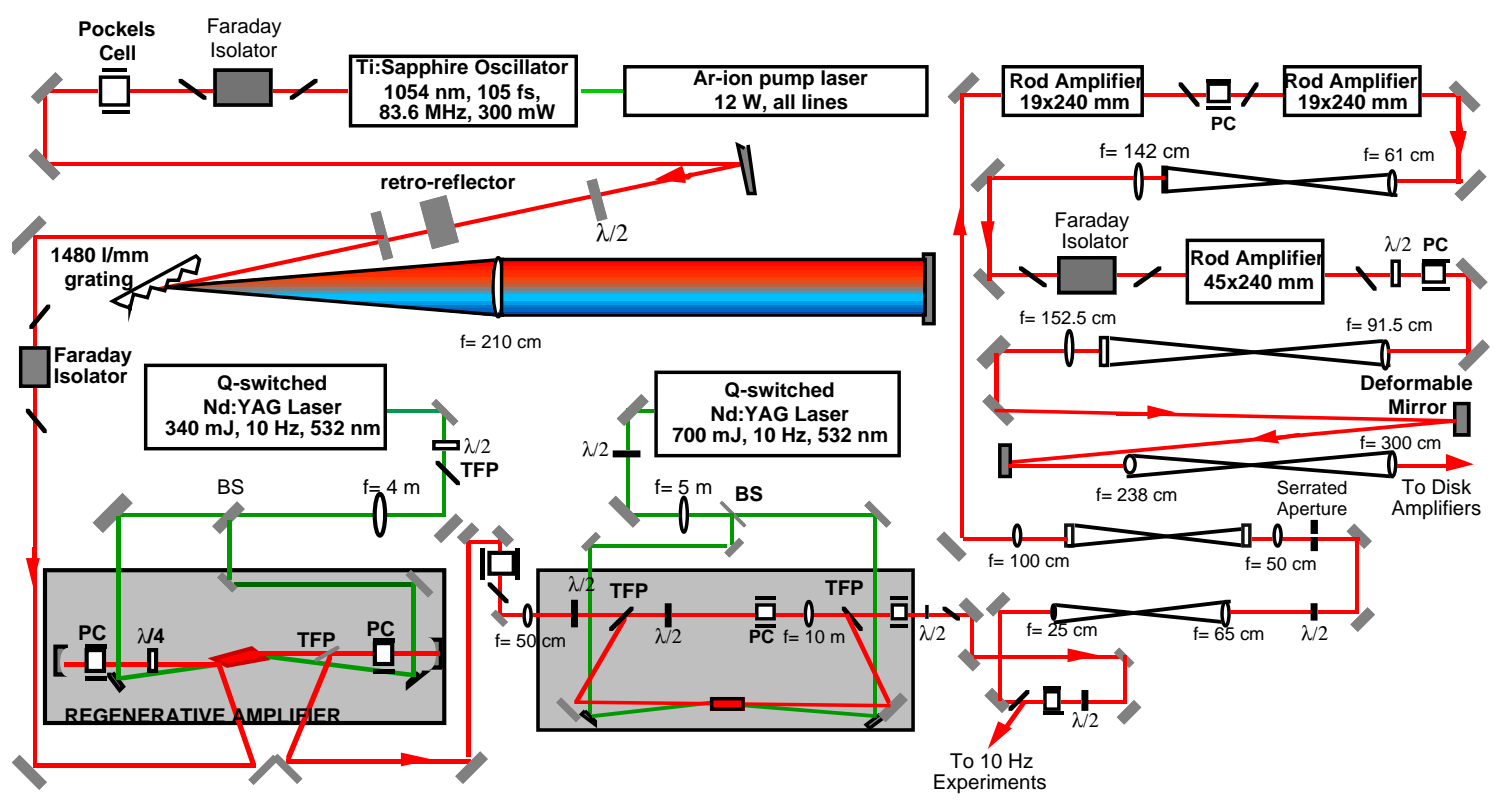

Figure 4.1. Front end of the 100TW laser system (and also the PW laser system). The final $9 \mathrm{~cm}$ disk amplifier for the 100TW laser is not shown. 
amplifiers; each type of Nd-doped glass has a different bandwidth, and the combination allows for a higher-bandwidth gain medium. The output of this system is a 20-45 Joule stretched pulse, also at $1.5 \mathrm{~ns}$ duration but with a smaller $4.7 \mathrm{~nm}$ bandwidth than the front end produced. This beam is focused through a spatial filter to improve beam quality, and then propagates into the compression chamber.

The compression chamber uses two $40 \mathrm{~cm}$-diameter gratings, separated by $4 \mathrm{~m}$, to compress the pulse. The chamber is kept at a vacuum of better than $10^{-5}$ torr, and has a throughput of $68 \%$. The final pulse length after compression is $400 \mathrm{fs}$. A small portion (4\%) of the beam is picked off, of which another $4 \%$ is sent to diagnostic stations that measure the near-field (unfocused) energy distribution, far-field (equivalent-plane focus) spot size, and autocorrelation (pulse width) on every shot. In order to allow sufficient cooling of the Nd:Glass amplifiers to allow a good focal spot, shots are limited to one every 45 minutes.

The output of this entire laser system is $12-30$ Joules of $1.06 \mu \mathrm{m}$ light in $400 \mathrm{fs}$. The peak intensity is $10^{7}$ times greater than the amplified spontaneous emission (ASE), which begins about $3 \mathrm{~ns}$ before the main pulse. An additional $400 \mathrm{fs}$ prepulse, reaching $\sim 10^{-3}$ of the peak intensity, arrives $\sim 2 \mathrm{~ns}$ early. The final focusing optic is an f/3 off-axis parabola, which produces a measured 15 micron FWHM focal spot (peak intensity of 4 $10{ }^{19} \mathrm{~W} \mathrm{~cm}^{-2}$ for 30 Joules). Interferometry measurements [58] and simulations show that the ASE and prepulse create an underdense plasma in front of the target with a scalelength on the order of $10 \mu \mathrm{m}$. In this plasma the intense laser may experience further self-focusing $[59,60]$. 


\subsubsection{Description of Experiment}

In the experiment the p-polarized laser light was incident at 25 degrees to the target normal. Figure 4.2 shows a sketch of the target makeup and geometry. The front (laser-incident) layer of the target was a 6 by $8 \mathrm{~mm}$ rectangular foil of various materials $(\mathrm{CH}, \mathrm{Al}$, or $\mathrm{Cu})$ with a mass per unit area ranging from .02 to $.45 \mathrm{~g} / \mathrm{cm}^{2}$. The middle layer of the target was a smaller foil ( 5 by $8 \mathrm{~mm}$ ) of $50 \mu \mathrm{m}$-thick Molybdenum. Electrons produced in the front layer transported into this Mo layer, knocking out inner-shell electrons and creating $17.5 \mathrm{keV} K_{\alpha} \mathrm{x}$-rays. Finally, a layer of $1 \mathrm{~mm}$ thick $\mathrm{CH}$ ( 7 by 10 $\mathrm{mm})$ covered the back of the target, which protected the Mo layer from electrons that might return to the target (pulled back by electrostatic forces). This $\mathrm{CH}$ layer stopped electrons with energies below $300 \mathrm{keV}$ (550keV attenuation for a double-pass), while

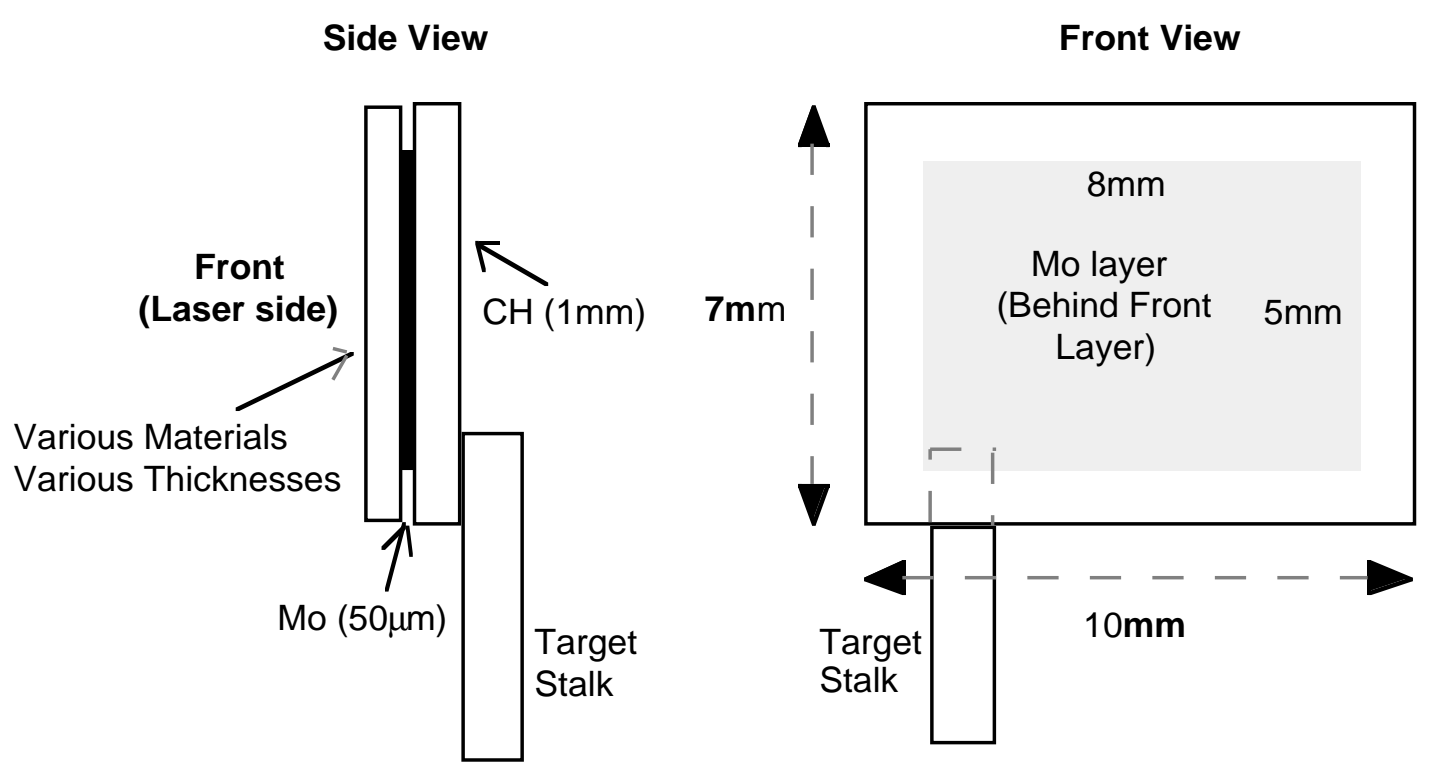

Figure 4.2. A schematic of the buried-layer target used in these experiments, and also those in Chapter 5. The laser is incident on the front side of the target, and $25^{\circ}$ to the target normal. The CCD detector is situated on the rear side of the target. 
having a negligible effect on the $17.5 \mathrm{keV}$ x-rays. This layer was found to lower the Mo $K_{\alpha}$ x-ray signal by a factor of $\sim 2$, which indicated that most of the $K_{\alpha}$ radiation was produced by electrons, and not x-rays.

This observation agrees with ITS simulations, which calculates the photo-pumped $K_{\alpha} \mathrm{x}$-rays in our targets to typically be $10 \%$ of the total, with the remaining x-rays pumped by the electrons. However, ITS only takes into account the secondary x-rays produced by the fast electrons; it does not account for any thermal x-rays from the laser focal spot. Fortunately, there are several pieces of evidence that the characteristic 17.5 $\mathrm{keV}$ Mo $K_{\alpha}$ photon energy is significantly greater than that of the x-rays produced by the thermal plasma around the laser focus. Spectroscopic measurements of an Al layer buried under a very thin layer of $5 \mu \mathrm{m} \mathrm{CH}$ showed a thermal plasma temperature of 300$600 \mathrm{eV}$ in separate experiments with the same laser [61]. Further evidence that thermal xrays were unimportant was that the $20-30 \mathrm{keV}$-ray spectrum was very similar from both the front and back of pure $\mathrm{Al}$ and $\mathrm{Cu}$ targets which were optically thick in this energy range. This signified that these $\mathrm{x}$-rays were predominantly bremsstrahlung photons produced throughout the cold target, which could be accounted for in the ITS simulations.

$K_{\alpha} \mathrm{X}$-rays from the Mo layer were detected by a 16-bit CCD detector, situated 2.16 meters from the target and 45 degrees from the rear target normal. The CCD was filtered with $75 \mu \mathrm{m}$ of $\mathrm{Sn}$, limiting the x-ray flux and making it unlikely that two highenergy photons would be absorbed in the same pixel. The counts recorded on each pixel were proportional to the x-ray photon energy.

The CCD camera was absolutely calibrated with a Cd-109 (22 keV) source at two different occasions during the experiments. The two calibrations agreed to within $3 \%$ and allowed us to calculate the number of incident x-rays from the measured hits on the 
camera. To scale the $22 \mathrm{keV}$ calibration to the $17.5 \mathrm{keV} K_{\alpha}$ x-rays, we assumed the detector response was proportional to the absorption of the $14 \mu \mathrm{m}$ thick Si CCD chip.

A statistical analysis was performed on each set of data to determine what fraction of the signal was obscured by double hits due to the lower energy x-ray continuum. For example, if a $17.5 \mathrm{keV}$ photon and a $800 \mathrm{eV}$ photon were both incident on the same pixel, the $18.3 \mathrm{keV}$ total would not appear to be a Mo $K_{\alpha} \mathrm{x}$-ray, although there was in fact one involved. This effect, if not corrected for, would lead to an undercounting of the $K_{\alpha} \mathrm{x}$-rays. In order to get a more accurate count, the fraction of the total pixels that registered below $450 \mathrm{eV}$ was determined for each shot $\left(\mathrm{F}_{450}\right)$. Then, an energy histogram was made of all pixels on the CCD and a $900 \mathrm{eV}$ window was drawn around the $17.5 \mathrm{keV} K_{\alpha}$ energy bin. All the hits in this window were summed, and the background x-rays were subtracted from the total (as determined by the $15 \mathrm{keV}-20 \mathrm{keV}$ continuum in the histogram). Finally, the remaining number was multiplied by $1 / \mathrm{F}_{450}$, in order to correct for the obscured hits.

The reason that a $900 \mathrm{eV}$ window must be used for binning is twofold. First, incident photons often lose a small (or sometimes large) portion of their energy to neighboring pixels. Therefore, even with an ideal monochromatic source, there will be a spread of energies in the pixel readings below $17.5 \mathrm{keV}$. The calibration was calculated with a $900 \mathrm{eV}$ window, so the data must be taken in the same manner. An additional energy spread occurs due to the background noise not present in the calibration; a large percentage of pixels are hit by a $100 \mathrm{eV}$ photon or greater. The method presented above is not perfect: a $17 \mathrm{keV}$ pixel will require a $950 \mathrm{eV}$ photon to raise it out of the counted window, while a $17.9 \mathrm{keV}$ pixel will only require a $50 \mathrm{eV}$ photon. Because the average pixel in the bin $(17.5 \mathrm{keV})$ is $450 \mathrm{eV}$ below the edge of the window, $\mathrm{F}_{450}$ is the appropriate background correction factor. 
In this manner, the number of $K_{\alpha} \mathrm{x}$-ray hits on the CCD is achieved for each shot. This number is normalized to the solid angle of the CCD chip, the filtering in front of the camera, the energy in the laser pulse and the above-mentioned calibration of the CCD camera. The final result is the number of $K_{\alpha} \mathrm{x}$-rays per Joule and per steradian emitted from the target, in the direction of the CCD camera. It is this number that must be accurately modeled with ITS in order to determine the parameters of the electron spectrum which produced the $K_{\alpha}$ X-rays.

\subsubsection{ITS Modeling}

Although ITS is a powerful and flexible code for calculating $K_{\alpha} \mathrm{x}$-ray yields from a given electron spectrum, it is more complicated to reverse the process and calculate the electron spectrum from a given set of $K_{\alpha} \mathrm{x}$-ray yields. One difficulty is the assumed energy distribution of the electrons. In general, the electron source was assumed to take the form of a Maxwellian energy distribution, which has been seen in PIC simulations $[60,62]$ and in experiments [50,54].

However, as there is no intrinsic reason why the electrons should be Maxwellian, ITS simulations were also performed for other possible distributions. For a relativistic Maxwellian, the mean electron energy $E_{o}$ ranges from $3 / 2 \mathrm{kT}$ (non-relativistic electrons) to $3 \mathrm{kT}$ (highly relativistic electrons); the exact relationship between average energy and temperature is shown in Figure 4.3. Comparing this to the case of a purely exponential spectrum $f(E)=\exp (-E / k T)$, for which $E_{O}=k T$, the ITS results were entirely consistent to within $10 \%$ for these different spectra, provided that $\mathrm{E}_{\mathrm{o}}$ (not kT) was kept constant. This demonstrated that this technique is not sensitive to the tail of the electron distribution (the slope of which determines kT), but rather to the mean-energy bulk of the distribution.

For this reason, the results are not directly comparable to some previous measurements of 
bremsstrahlung x-rays or the high-energy electron tail [54]. However, this technique is appropriate for absolute conversion efficiency measurements which depend on the mean energy $\mathrm{E}_{\mathrm{O}}$. 


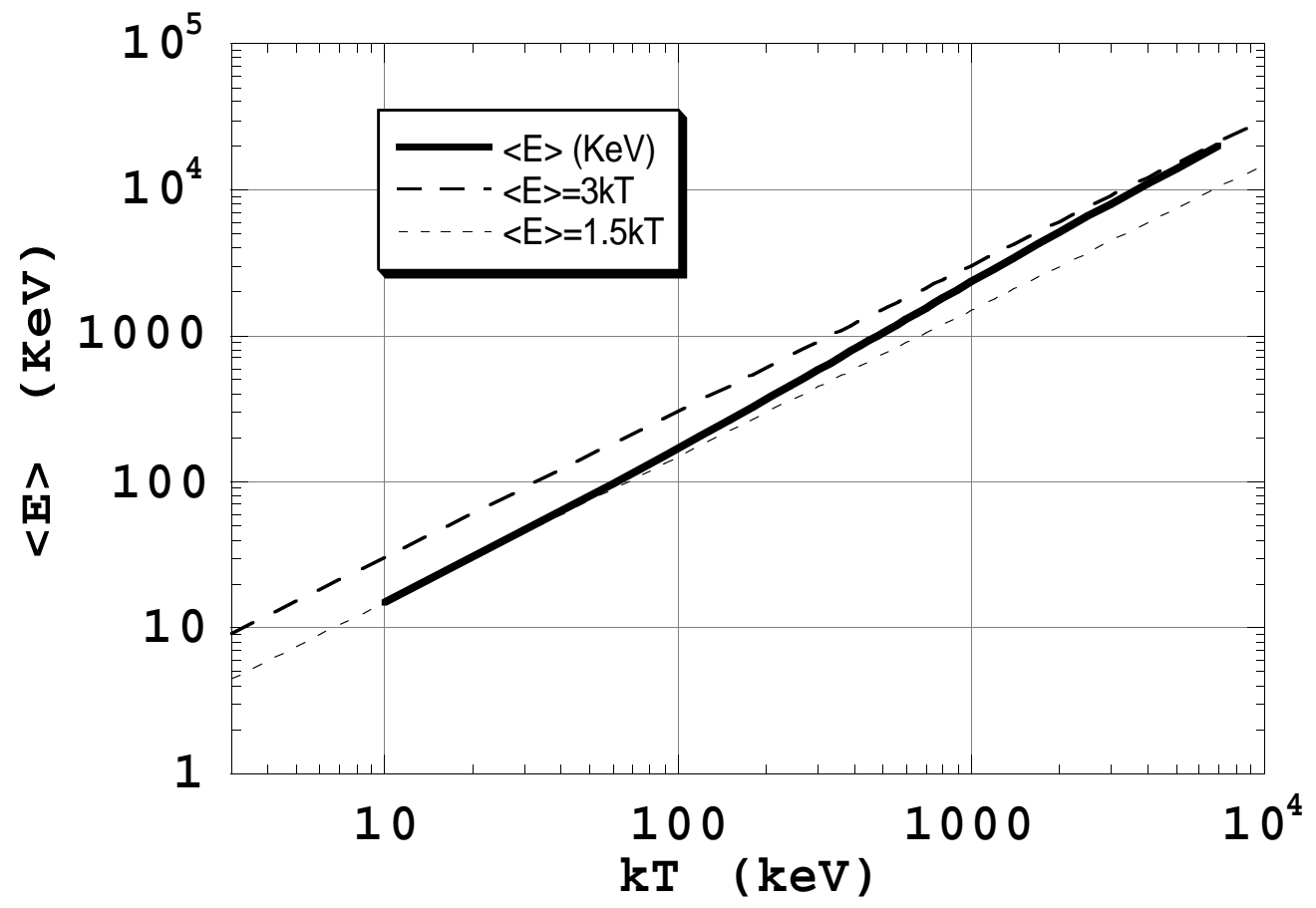

Figure 4.3. The relationship between mean electron energy $\langle\mathrm{E}\rangle$ and temperature $\mathrm{kT}$ is shown for a relativistic Maxwellian energy distribution. At low (sub-relativistic) energies, the usual relationship $\langle\mathrm{E}\rangle=1.5 \mathrm{kT}$ holds; at very relativistic energies, $\langle\mathrm{E}\rangle$ approaches $3 \mathrm{kT}$.

Another assumption required in ITS is the cone-angle of the electrons. For now the assumption will be that the electrons spray forward isotropically from the laser focus into a full hemisphere; this will be discussed further in section 4.2.2. ITS also assumes that the electrons transport through cold material, and the code ignores collective effects such as self-consistent magnetic [60,63] and electrostatic fields [64-66]. Because of the complexity of the physics involved, the use of ITS is not intended to fully model the experiment but is used as a benchmark for interpreting the data. Further modeling and analysis will be required to account for these effects.

\subsection{Results and Analysis}




\subsubsection{Electron Energies and Conversion Efficiencies}

The Mo $K_{\alpha}$ yields from the targets with Aluminum front layers are presented in Figure 4.4, along with the best fits from the ITS code. The slope of the data (on a log plot) is sensitive to the mean energy $E_{o}$ of the electrons, while the absolute magnitude yields the laser-to-electron conversion efficiency $\eta$. Error bars were computed from a combination of counting errors and fluctuations in the background x-ray noise.

The data are fit with a series of ITS runs which computed the $K_{\alpha} \mathrm{x}$-ray yield as a function of the transport layer thickness for a given electron mean-energy $E_{0}$. The conversion efficiency $\eta$ was found to minimize the chi-squared of the fit per degree of freedom. The data at an intensity of $210^{19} \mathrm{~W} \mathrm{~cm}^{-2}$ are fit by an ITS run with $E_{0}=330 \mathrm{keV}(\mathrm{kT}=170 \mathrm{keV})$ and $\eta=31 \%$. For this fit, the chi-squared is reasonably small

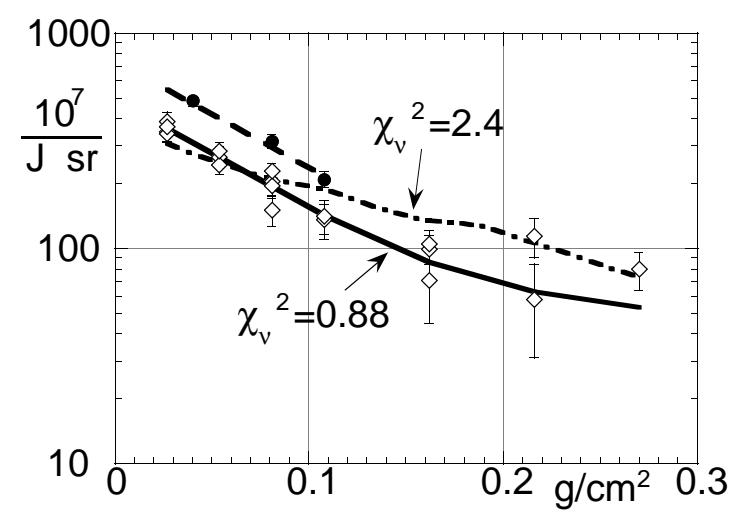

Figure 4.4. $K_{\alpha}$ signal from the $\mathrm{Al}$ targets, in units of $10^{7} \mathrm{x}$-rays per incident Joule and per steradian, plotted against areal mass of the Aluminum front layer of the target. The solid circles are experimental data at intensities of $410^{19} \mathrm{~W} \mathrm{~cm}^{-2}$, empty diamonds are data at $210^{19} \mathrm{~W} \mathrm{~cm}^{-2}$. The solid line is an ITS fit with mean energy $E_{0}=330 \mathrm{keV}$ and conversion efficiency $\eta=31 \%$. The dash-dot line is $E_{0}=640 \mathrm{keV}$ and $\eta=30 \%$ The dashed line is $E_{0}=330 \mathrm{keV}$ and $\eta=47 \%$. All values of $\eta$ are multiplied by 0.7 if the electrons are assumed to be directed in a $30^{\circ}$ half-angle cone. 
(0.88). An ITS run for $\mathrm{E}_{\mathrm{O}}=640 \mathrm{keV}(\mathrm{kT}=300 \mathrm{keV})$ is also shown, although the chi-squared of this fit is much larger (2.4). Three data points at an intensity of $410^{19} \mathrm{~W} \mathrm{~cm}^{-2}$ show a higher $\eta(47 \%)$ but roughly the same mean energy.

Figure 4.5 shows the experimental results for $\mathrm{CH}$ and $\mathrm{Cu}$ targets at a laser intensity of $210^{19} \mathrm{~W} \mathrm{~cm}^{-2}$. The $\mathrm{CH}$ targets produced the smallest signal, corresponding to $E_{0}=120 \mathrm{keV}$ and $\eta=29 \%$. The $\mathrm{Cu}$ data have the largest error bars, due to higher $\mathrm{x}$-ray noise, but are best fit by $\mathrm{E}_{\mathrm{O}}=640 \mathrm{keV}$ and $\eta=29 \%$. Higher and lower energy fits to the $\mathrm{Cu}$ data are shown as well. The lower-intensity Al data from Figure 1 are at the same intensity; recall they were fit by $E_{0}=330 \mathrm{keV}$ and $\eta=31 \%$. The data show a change of mean electron energy with target material, although the conversion efficiencies remain roughly constant.

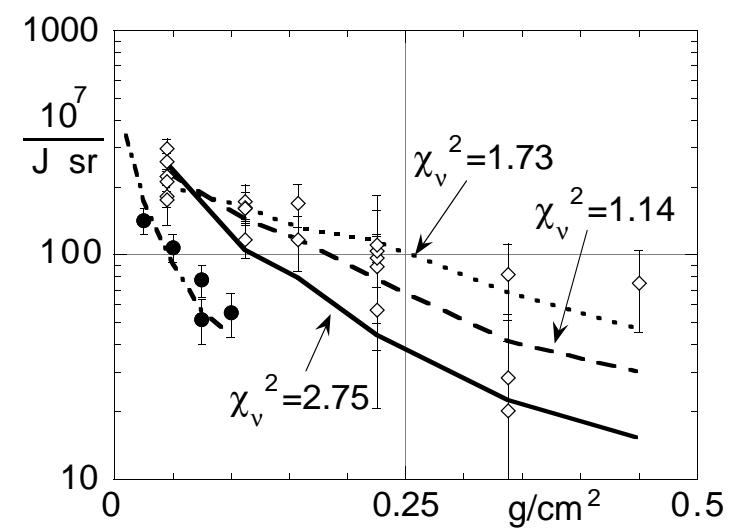

Figure 4.5. $K_{\alpha}$ signal from the target, same units as Fig. 1. The solid circles are data from $\mathrm{CH}$ front-layer targets, empty diamonds are data from $\mathrm{Cu}$ targets, both at $210^{19} \mathrm{~W}$ $\mathrm{cm}^{-2}$. The solid line is an ITS fit in $\mathrm{Cu}$ with mean energy $\mathrm{E}_{\mathrm{O}}=330 \mathrm{keV}$ and conversion efficiency $\eta=30 \%$. The dashed line is $E_{0}=640 \mathrm{keV}$ and $\eta=29 \%$ The dotted line is $\mathrm{E}_{\mathrm{O}}=1040 \mathrm{keV}$ and $\eta=31 \%$. The dash-dot line is an ITS fit in $\mathrm{CH}$ for $\mathrm{E}_{\mathrm{o}}=120 \mathrm{keV}$ and $\eta=29 \%$. All values of $\eta$ are multiplied by 0.7 if the electrons are assumed to be directed in a $30^{\circ}$ half-angle cone. 


\subsubsection{Electron Directionality}

The assumption that the electrons are spraying into a full hemisphere might artificially increase the apparent conversion efficiency. To measure the directionality of the electrons, a stainless steel razor blade of $750 \mu \mathrm{m}$ thickness (and $1 \mu \mathrm{m}$ rms flatness) was placed $30 \mathrm{~cm}$ from the back of the target, in line with the CCD detector. This created a 1D penumbral image of the x-ray source on the CCD [50,67] with a magnification of 6 . Using this configuration, $210^{19} \mathrm{~W} \mathrm{~cm}^{-2}$ laser pulses were shot at some of the previously described targets; $\mathrm{CH}$ front layers (varied thicknesses), Mo middle layers, and optional $\mathrm{CH}$ back layers to prevent electron double-hits. The lack of measured $\mathrm{x}$-rays above $6 \mathrm{keV}$ from pure $\mathrm{CH}$ targets, along with the opacity of the razor blade to $\mathrm{x}$-rays under $20 \mathrm{keV}$, meant that the size of the 6-20keV x-ray source was a good measure of the where the electron beam intersected the Mo layer. Varying the depth of the Mo gave an estimate of the electron cone-angle.

A sample data set for a single shot is shown in Figure 4.6. This data shows the number of 6-20keV x-rays as plotted against linear position on the CCD camera. Geometrical considerations (assuming a point source of electrons which create a circular source of x-rays in the Mo layer) predict that the data should follow an arctangent function. The best arctangent fit is shown, which fits the data well. The maximum slope of this fit determines the size of the $\mathrm{x}$-ray source; therefore a derivative is required to extract this parameter.

Figure 4.7 shows this measured spot size graphed against the buried depth of the $50 \mu \mathrm{m}$ Mo layer, and compares it to ITS calculations of the predicted measurements for electron beams with $30^{\circ}$ and $90^{\circ}$ half-cone angles. The large error bars result from the derivative that is required to extract the spot size from the data. For Mo layers buried 100 to 250 microns into the target, the data roughly correspond to an electron cone half-angle of $90^{\circ}$, a full hemisphere. However, for the thicker targets the x-ray source corresponds 


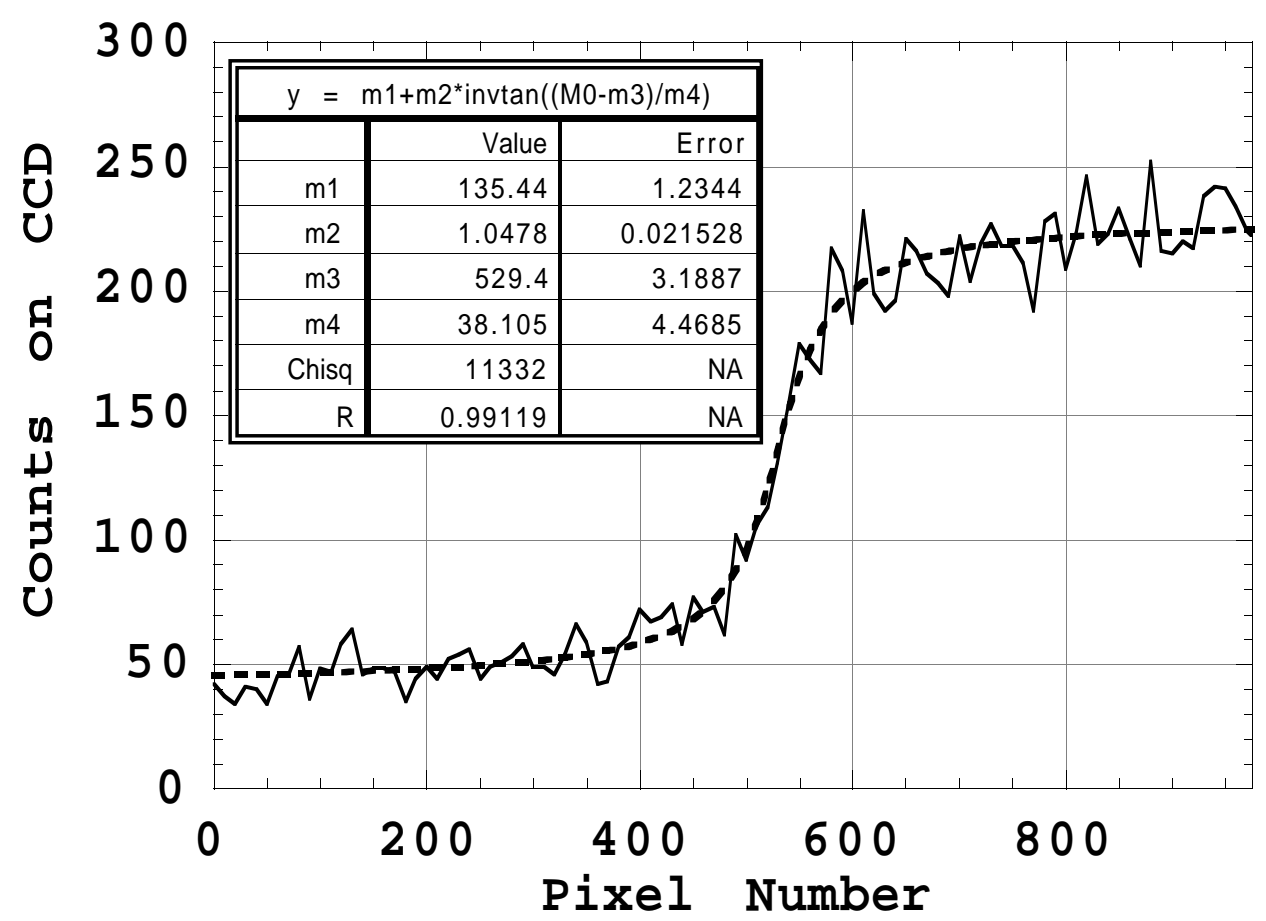

Figure 4.6. The number of single-pixel CCD hits (between $6 \mathrm{keV}$ and $20 \mathrm{keV}$ ) is plotted against position on the CCD camera, from a target shot with a $100 \mu \mathrm{m}$ layer of $\mathrm{CH}$ in front of the $50 \mathrm{Mo \mu m}$ layer. The data is fit with an arctangent function (Equation shown), and the maximum slope of the fit (parameter m4) determines the FWHM of the $\mathrm{x}$-ray source. For this case, the FHWM of the x-ray spot is $295 \pm 35 \mu \mathrm{m}$.

closer to an electron beam of a $30^{\circ}$ half-cone angle. Although the error bars are large, these data suggest some beaming of the high energy electrons $(>200 \mathrm{keV})$ that penetrate through the thicker targets. The bulk of the lower-energy electrons seem to be spraying into a full hemisphere. Using ITS to recalculate the conversion efficiencies based on a $30^{\circ}$ half-angle electron source lowers $\eta$ to 0.7 of the above-quoted $90^{\circ}$ values. The mean electron energies were not affected. 


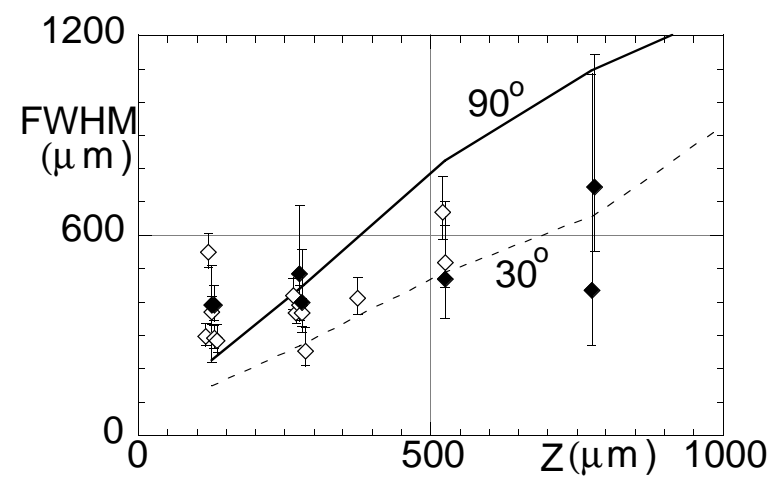

Figure 4.7. The measured size (FWHM in $\mu \mathrm{m})$ of the x-ray source is plotted against thickness of the front $\mathrm{CH}$ layer in $\mu \mathrm{m}$. Solid diamonds are from targets with a back layer of $1 \mathrm{~mm} \mathrm{CH}$, empty diamonds had no back $\mathrm{CH}$ layer. The solid line is an ITS fit of the expected results from an unbeamed electron source ( $90^{\circ}$ half-angle). The dashed line is an ITS simulation of an electron source with a $30^{\circ}$ half-angle.

Applying this beaming effect to the earlier data, our measurements correspond to $\eta=21 \% \pm 5 \%$ for all materials at a laser intensity of $210^{19} \mathrm{~W} \mathrm{~cm}^{-2}$, and $\eta=33 \% \pm 5 \%$ for the high-intensity $\left(410^{19} \mathrm{~W} \mathrm{~cm}^{-2}\right)$ shots on $\mathrm{Al}$ targets. Further intensity scaling will be discussed in Chapter 5.

\subsubsection{Analysis}

The average-energy measurements from section 4.2.1 seem to vary with target material rather than intensity. Our data show that the $\mathrm{Cu}$-produced electrons are the most penetrating, although the error bars on the measurements still allow the possibility that the $\mathrm{Al}$ and $\mathrm{Cu}$ spectra could be equivalent. The $\mathrm{CH}$ electrons are less penetrating and apparently colder, although they seem to have roughly the same conversion efficiency as the $\mathrm{Al}$ and $\mathrm{Cu}$ targets. The conversion efficiency in $\mathrm{CH}$, however, has an additional systematic error because the range of an $E_{0}=120 \mathrm{keV}$ electron is smaller than the typical 
target thickness, which means that the experiments in $\mathrm{CH}$ do not measure the bulk of the electron distribution as is done in $\mathrm{Al}$ and $\mathrm{Cu}$. Instead the experiment may be primarily measuring the hot-electron tail, and inferring a conversion efficiency through the assumption of a Maxwellian spectrum. For this reason, the conversion efficiencies in Al and $\mathrm{Cu}$ should be read as more accurate than in $\mathrm{CH}$, although there are still clear difference between the materials.

As previously discussed, ITS cannot factor in any collective effects that would arise from the strong electron currents. Bell, Davies, and collaborators have pointed out that strong material-dependent effects may result from differences in target conductivity $[65,66]$. Conductivity has long been known to play an important role in shielding the resistive electrostatic field via a return current [64]. To explore some of these effects 1-D LASNEX [37] simulations were performed in which a high energy Maxwellian distribution of electrons transport from the center of a solid density sphere. The return current, heating, conductivity, and electrostatic fields are calculated self-consistently, and show a $\sim 40 \%$ loss of electron energy to resistive electrostatic fields. Other simulations have put this number at 30\% [66]. This loss implies that our measurements of the fast electrons must be lower bounds on the original electron parameters, ideally requiring a correction for electrostatic effects. However, electrostatic effects cannot fully explain the observed material-dependence because the conversion efficiency is not lowered by the same factor as $E_{0}$ in the different target materials.

Another difference between the target materials is the underdense plasma that the ASE and prepulse form in front of the target. 2-D calculations with LASNEX show a larger stand-off between the critical and solid densities in $\mathrm{CH}(40 \mu \mathrm{m})$, compared to $\mathrm{Al}$ $(22 \mu \mathrm{m})$ and $\mathrm{Cu}(18 \mu \mathrm{m})$. This difference is due to the variation in the $\mathrm{Z}$ of the target. Recall from Eqn. 4.5 that the peak unshielded current scales with the square root of the density; without a steep density gradient it is possible that the huge magnetic fields could 
pinch off much of the current before it propagated into the solid-density region. This effect might be even more material-dependent because target conductivity can also affect the strength and quickness of the return current deeper in the target. But again, this explanation cannot explain why the conversion efficiency would not seem to change with target material.

The data seems to be pointing to an initial electron spectrum that is materialdependent, not merely material-dependent propagation efficiencies; this is the only way to explain the different energies but same conversion efficiencies in the various targets. But this is a surprising and unexpected result; in all cases, the interaction happens in a fully ionized plasma at critical density. One possibility is that the different pre-plasma made by the interaction of the laser pre-pulse with the different materials affect the intensity distribution of the laser through filamentation instabilities and relativistic selffocusing $[59,60]$. This would be a mechanism that might change the original electron spectrum based on the target material, but it does not seem to be a consistent explanation. For example, when the intensity was increased from $210^{19} \mathrm{Wcm}^{-2}$ to $410^{19} \mathrm{~W} \mathrm{~cm}^{-2}$ in Al, the conversion efficiency did not remain constant but increased. Therefore if there was a different intensity for the different targets one might expect this same change in conversion efficiency, rather than a change of average electron energy.

The solution to this dilemma might lie at the origin the source electrons. If the electrons originated in the laser focal spot, then the original spectrum should be entirely laser-dependent: the target material should not matter much at all. But in our experiment the return current serves as the primary source of the hot electrons, because the number of fast electrons we infer from our results is much greater than the number of electrons in a cubic laser spot size of critical density plasma. Our results imply that the laser creates $10^{13}(\mathrm{Cu})$ to $10^{14}(\mathrm{CH})$ electrons, while there are only $410^{11}$ electrons in a 20 -micron 
sphere of critical density plasma (for $1 \mu \mathrm{m}$ light). Even fewer electrons can leave a spot of this size $\left(\sim 10^{10}\right)$ before $\mathrm{MeV}$-scale electrostatic potentials are created.

Therefore most of our accelerated electrons must originate in the overdense target, pulled into the laser-dominated region by electrostatic forces. The electrons may gain energy in this manner because the pondermotive force of the laser (or $\mathrm{JxB}$ force) is not constant in time; electrons pulled in at the right phase can be kicked out again with a net gain of energy. But the details of this process are tremendously complicated and no doubt depend a great deal on the phase and energy of the incident electrons.

Despite the complexity, this provides a mechanism by which the target material can affect the original spectrum of the electron and not merely their propagation. The different conductivities and densities will affect the return current of electrons. This return current then supplies the source electrons which will be accelerated by the laser, but the precise energy gain and conversion efficiency will be determined in part by the details of the return current. Therefore the original electron spectrum can depend on the conductivity and density of the target material, despite the fact that the primary interaction occurs in a critical density plasma in all cases. Our data supports this hypothesis, although further experiment and simulations would be necessary to place it on firmer ground.

In summary, we have demonstrated a 20\%-30\% conversion efficiency from laser energy into forward-propagated electrons in solid targets. This efficiency seems to be a function of intensity but not target material. A material-dependence on electron temperature has been demonstrated for the first time at intensities above $10^{19} \mathrm{~W} \mathrm{~cm}^{-2}$. Interestingly, the material-dependence seems to affect the original electron spectrum and not merely the propagation characteristics. Chapter 6 contains further discussion of these results and their relevance to the fast ignitor fusion concept. 


\section{Chapter 5}

\section{Laser-Solid Interactions with the Petawatt Laser}

\subsection{Experimental Set-up}

\subsubsection{Description of Laser}

The Petawatt laser at LLNL [68] is currently the most powerful single laser beam on Earth, comparable to the planned power of the entire 192-beam NIF facility (for a much shorter period of time). The front end of the laser system is the same front end as the 100TW laser, described in section 4.2.1 and shown in Figure 4.1. From the front end, the chirped $1.06 \mu \mathrm{m}$ wavelength pulse is sent through one of the ten NOVA amplifier chains, as detailed in Figure 5.1. This chain consists of ever-larger disk amplifiers, producing a maximum of $\sim 800 \mathrm{~J}$ after the $31.5 \mathrm{~cm}$ disk amplifiers. Spatial filter pinholes improve the beam quality, clipping off the edges of the focused beam and reducing the beam energy to $\sim 700 \mathrm{~J}$.

This beam is then propagated to the large compression chamber (Figure 5.2). Compressing the pulse to a full Petawatt requires a pair of diffraction gratings, each $75 \mathrm{~cm}$ in diameter (after these experiments were performed the grating size was increased to $1 \mathrm{~m})$. As in the $100 \mathrm{TW}$, the two gratings are contained in a large vacuum chamber, at a pressure of $10^{-5}$ torr. The throughput of the compressor is $\sim 80 \%$, allowing $550 \mathrm{~J}$ in a pulse may be compressed to 580fs (FHWM). Typical shot energies lie in the 200-400 Joule range, and this chapter will discuss shots in which the pulse length was 5-20ps, although 0.5 ps has also been achieved. 


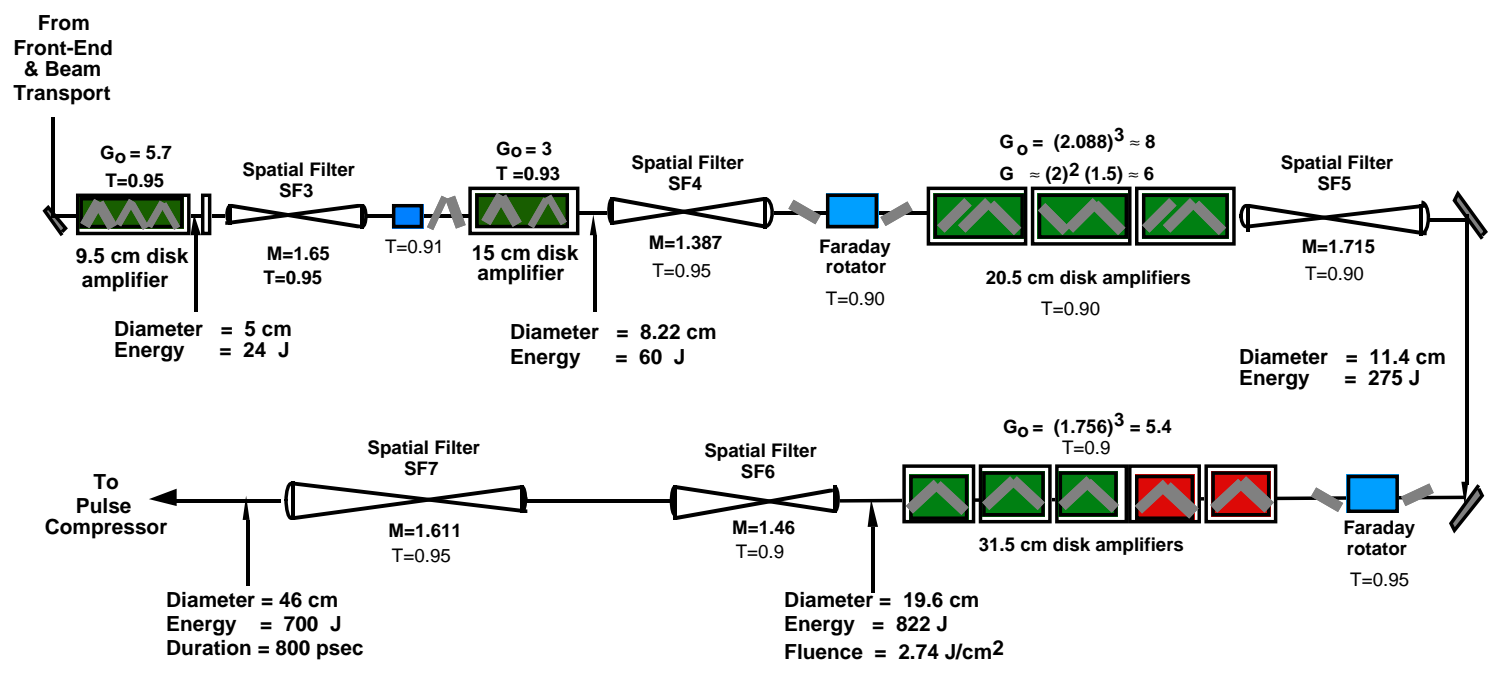

Figure 5.1. The Nova amplifer chain in which the Petawatt pulse is propagated. The input pulse to this chain is the same as the output pulse from Figure 4.1.

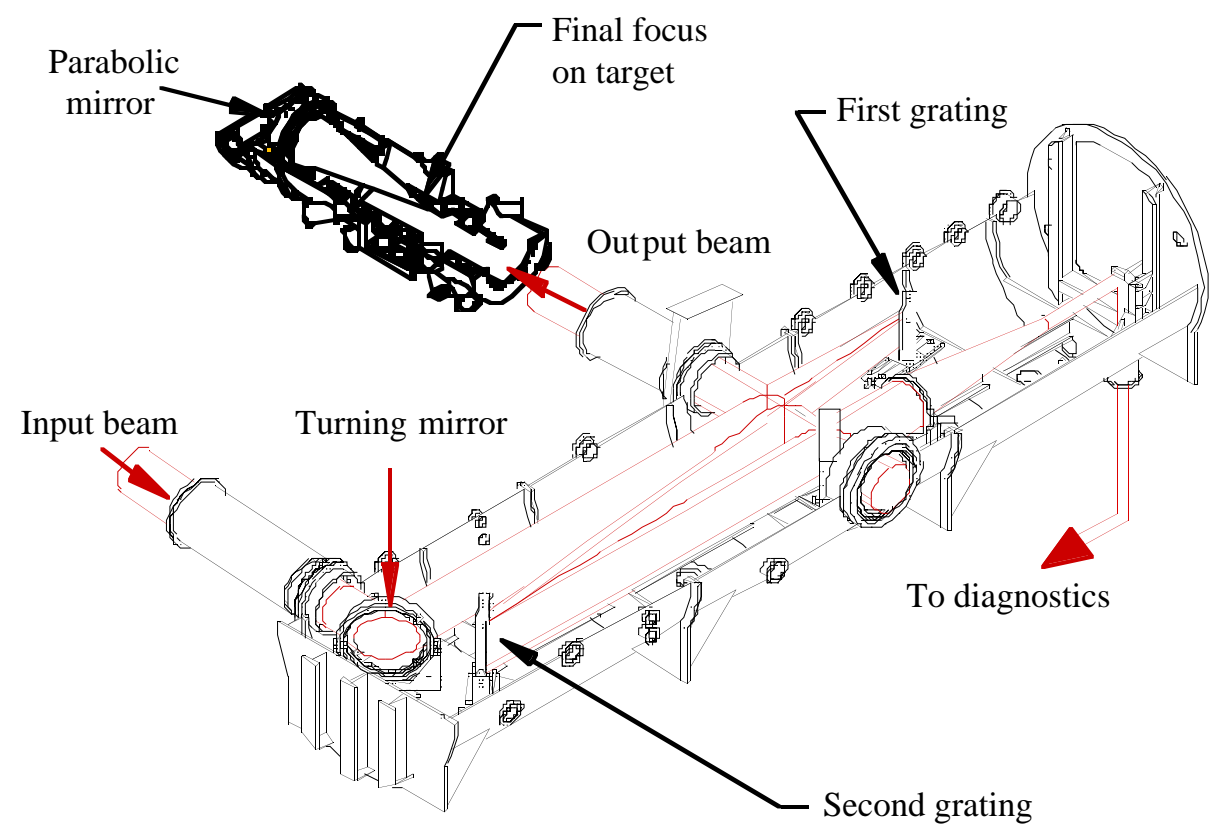

Figure 5.2. The compression chamber and target chamber for the Petawatt system. The target chamber utilizes an on-axis parabola to focus the beam in the center of the cylindrical target chamber. 
The Petawatt target chamber contains an on-axis parabola that focuses the laser back toward the compression chamber. A circular beam block prevents unfocused light from hitting the target on the first pass, and a large fused-silica debris shield protects the parabola on the shots discussed in this chapter.

The focal spot of the Petawatt beam was worse than on the 100TW, largely as a result of the additional amplification stages. Low-energy shots were measured to have a $20 \mu \mathrm{m} \times 40 \mu \mathrm{m}$ FWHM, and although this parameter could not be monitored on most target shots, the higher energies might have been worse. The same ASE and prepulse levels from the 100TW were also present, but the preplasma was likely dominated by the short prepulse $\left(10^{-3}\right.$ to $10^{-2}$ of the peak intensity, $\sim 3 \mathrm{~ns}$ before main pulse) because of the lower peak intensity $\left(\sim 10^{18} \mathrm{~W} / \mathrm{cm}^{2}\right)$ of the $20 \mathrm{ps}$ Petawatt shots.

The shot rate on the Petawatt was much lower than that of the 100TW; only 3-4 shots per day instead of the 8-12 possible on the 100TW. Fully compressed shots (580fs) were not available for these experiments, as those short pulse intensities cannot pass through the parabola debris shield without inducing phase distortions and thereby destroying the final laser focus. Therefore the highest intensities possible for these shots was still less than the lowest intensity shots performed on the 100TW laser. Therefore the purpose of these experiments was not to extrapolate to higher intensities, but rather to repeat the previous experiment at higher total energies, and therefore higher total electron flux.

\subsubsection{Description of Experiment}

As in the previous chapter, a buried-layer $K_{\alpha}$ technique was used to measure the laser-produced electrons while still in the target. The same (calibrated) CCD camera from the previous 100TW experiments was brought to the Petawatt chamber and mounted $3 \mathrm{~m}$ from target chamber center. 
Some differences in the diagnostic filtering were required due to the high levels of x-rays produced by the Petawatt laser. As mentioned in Chapter 4, sufficiently energetic photons can induce $K_{\alpha}$ radiation in the filter materials, and therefore the $\mathrm{Sn}$ filters in front of the CCD camera became an unwanted source in the $5-25 \mathrm{keV}$ energy range when irradiated by the much higher x-ray energies from the target. Removing these Sn filters actually resulted in somewhat fewer hits on the CCD camera, as the thin Si chip of the camera was transparent to the very high energy x-rays but not the $25 \mathrm{keV} K_{\alpha}$ radiation from the Sn filters.

As some filtering was still required, the $\mathrm{x}$-ray signal was attenuated with Aluminum plates situated at the window of the target chamber, $1 \mathrm{~m}$ from the target. These lower-Z filters did not produce as many energetic $\mathrm{x}$-rays, and because of the $\sim 2 \mathrm{~m}$ distance between the filters and the CCD camera, the secondary X-rays were further attenuated by geometric considerations. (Regardless, these x-rays were less important than the secondary radiation from the chamber walls). The final filtering allowed for measurable levels of $17.5 \mathrm{keV} K_{\alpha}$ radiation from the Mo layer, sufficiently above the background x-ray counts.

Further differences from the earlier 100TW experiment were imposed by the geometry of the target chamber; although p-polarization was still used, the targets were now shot at a $45^{\circ}$ angle of incidence, as opposed to the $25^{\circ}$ angle that characterized the experiments on the 100TW. Several shots on the $100 \mathrm{TW}$ were performed at $45^{\circ}$, however, and the results were essentially identical to the $25^{\circ}$ shots. Therefore this new angle of incidence was not expected to significantly change the results of the experiment.

During the course of this experiment, a typical 16-hour run day on the Petawatt yielded 0-4 shots. In part this was due to the technical difficulties of bringing up a new, large laser system. Further difficulties in obtaining many shots arose because Petawatt operation interfered with 10-beam Nova operation, so that only 3-4 shot days were 
available each month. Because of the limited number of shots on the Petawatt laser, varying target materials was not an option. Instead, Aluminum was always used as the front layer. The targets were identical to the previous Al targets from Chapter 4 (Figure 4.2), with $50 \mu \mathrm{m}$ of Mo in the center and $1 \mathrm{~mm}$ of $\mathrm{CH}$ on the back to prevent electron reflux.

\subsection{Results}

\subsubsection{Data at 20ps pulse length}

Unlike the smooth-running and reproducible 100TW laser, the timing of these experiments corresponded with the Petawatt being a new and evolving laser facility. The result of this was both limited shots and varying laser energy (200-450J). The low total number of shots did not allow an extensive data set over any near-constant laser energy. Because the very concept of the buried-layer technique requires a laser that is reproducible from shot to shot, this posed some difficulties. As a result, the limited Petawatt data was primarily interpretable only by making assumptions not made in the previous chapter.

Our most extensive data set at a single set of laser parameters was four shots, all between $325-425 \mathrm{~J}$ of energy and at a 20ps pulse length. The power of these shots was therefore $\sim 20 \mathrm{TW}$, significantly less than the 40-80TW experiments discussed in the previous chapter. The intensity dropped even further $\left(\mathrm{I}=2.510^{18} \mathrm{~W} \mathrm{~cm}^{-2}\right)$, because of the larger spot size $(20 \mathrm{x} 40 \mu \mathrm{m}$, as opposed to $15 \mathrm{x} 15 \mu \mathrm{m})$. Still, this experiment probed high intensity effects at much larger time scales (and energies) than was done on the 100TW laser.

As mentioned above, all of the targets had Aluminum front layers of varying thickness. The $50 \mu \mathrm{m}$ Mo middle layer and $1 \mathrm{~mm} \mathrm{CH}$ back layer were identical to the targets shot in the previous experiments. 


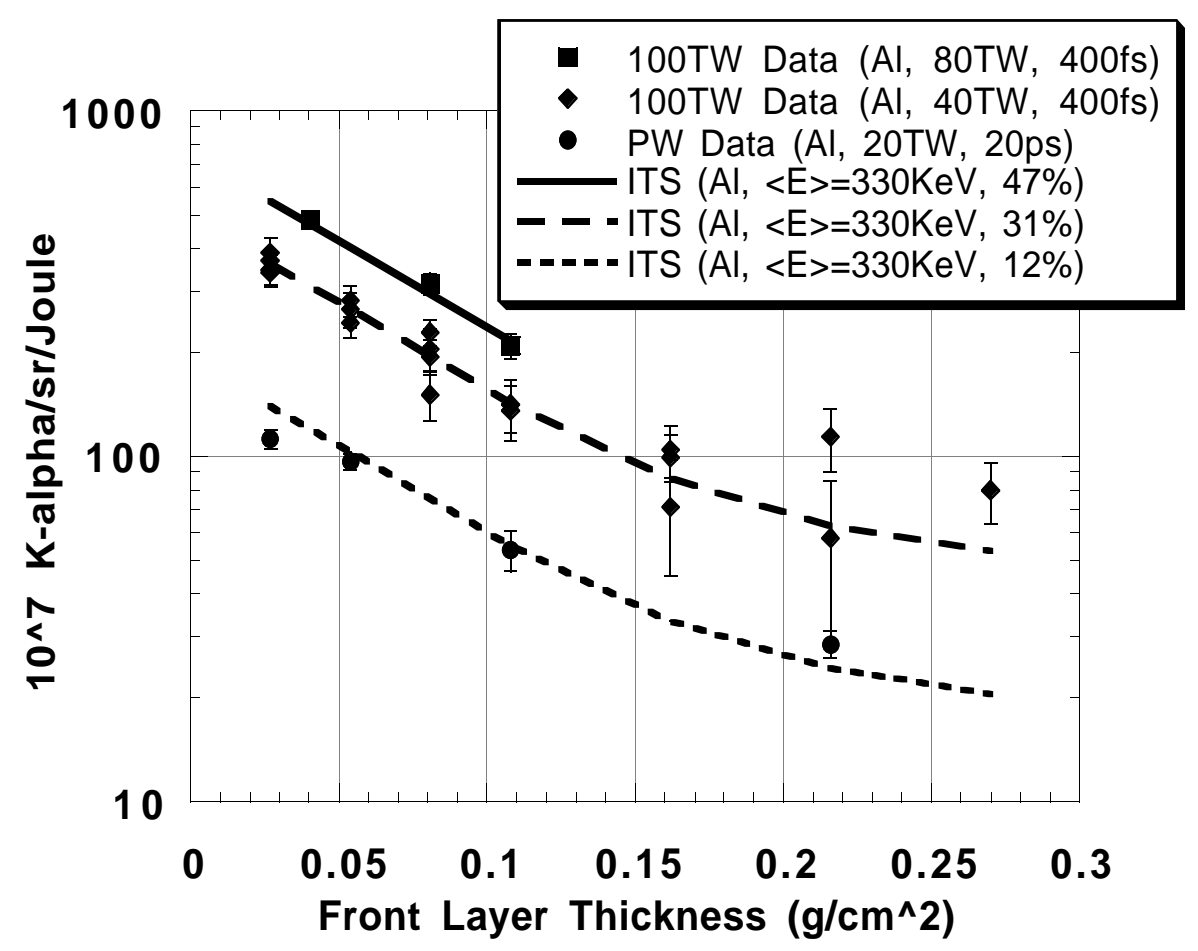

Figure 5.3. $K_{\alpha}$ signal from the $\mathrm{Al}$ targets, in units of $10^{7} \mathrm{x}$-rays per incident Joule and per steradian, plotted against areal mass of the Aluminum front layer of the target. The solid squares are experimental data at intensities of $410^{19} \mathrm{~W} \mathrm{~cm}^{-2}$, diamonds are data at 2 $10^{19} \mathrm{~W} \mathrm{~cm}^{-2}$, and circles are 20ps Petawatt shots at $210^{18} \mathrm{~W} \mathrm{~cm}^{-2}$. The lines are ITS fits with mean energy $E_{0}=330 \mathrm{keV}$ and various conversion efficiencies. All efficiencies are multiplied by 0.7 if the electrons are assumed to be directed in a $30^{\circ}$ half-angle cone.

The results of the four 20TW shots are shown in Figure 5.3, compared to the previous results in Aluminum targets from Chapter 4 . The $K_{\alpha}$ yields are normalized to the incident laser energy so that data from the different experiments are comparable. The conversion efficiency is much lower in the newer experiments (12\%), as might be expected from the lower intensity. This conversion efficiency assumes the electrons are spraying into a $2 \pi$ hemisphere; as in the previous chapter, a beam of $30^{\circ}$ half-cone angle can reduce the efficiency by a factor of 0.7 . More surprising, however, is the slope of the data; it is indistinguishable (within the error bars) from the slope of the higher intensity 
experiments. This is a surprising result, as the mean energy of the electron spectrum appears similar $\left(E_{0}=330 \mathrm{keV}\right)$ over very different intensities. Further discussion of this result will follow in the next section.

Two additional shots were taken at the 20ps pulse length, but they were lower energy shots $(\sim 200 \mathrm{~J})$ and therefore cannot be directly compared with the other data. They are not shown in Figure 5.3, as fitting a line to these two points would be very prone to error and would not likely lead to any meaningful results. Instead, they are included in Figure 5.4, and an additional assumption is required to infer much from these data points, as will be seen in the next section.

\subsubsection{Data at 5ps pulse length}

Four Petawatt shots were then taken at 5ps pulse length; the shortest pulse for which the large debris shield could be left in the beam path without distorting the laser focus. The energy for these four shots varied from (250-350J), which again makes direct analysis difficult as there were not many data points with the same parameters.

These four data points are shown in Figure 5.4, along with all six 20ps shots. Clearly they are difficult to interpret because the shot with the largest $K_{\alpha}$ yield does not correspond to the thinnest target (but rather to the highest energy shot). Because the focal intensity is not constant between shots, it must somehow be taken into account in analyzing this limited data.

At this point a simplifying assumption can be made that $E_{0}$ is independent of laser intensity. This is based on the observation (from Figure 5.3) that the average electron energy $E_{o}$ seems to be the same for a wide range of intensities. Although this result is not at all expected, it is not inconceivable. Recall the simple pondermotive energy scaling from Eqn. 2.8; plugging in the 20ps intensity $\left(\mathrm{I}=2.510^{18} \mathrm{~W} \mathrm{~cm}^{-2}\right)$ yields a hot electron temperature of $\mathrm{T}_{\text {hot }}=340 \mathrm{keV}$. Even this number is significantly higher than the measured 


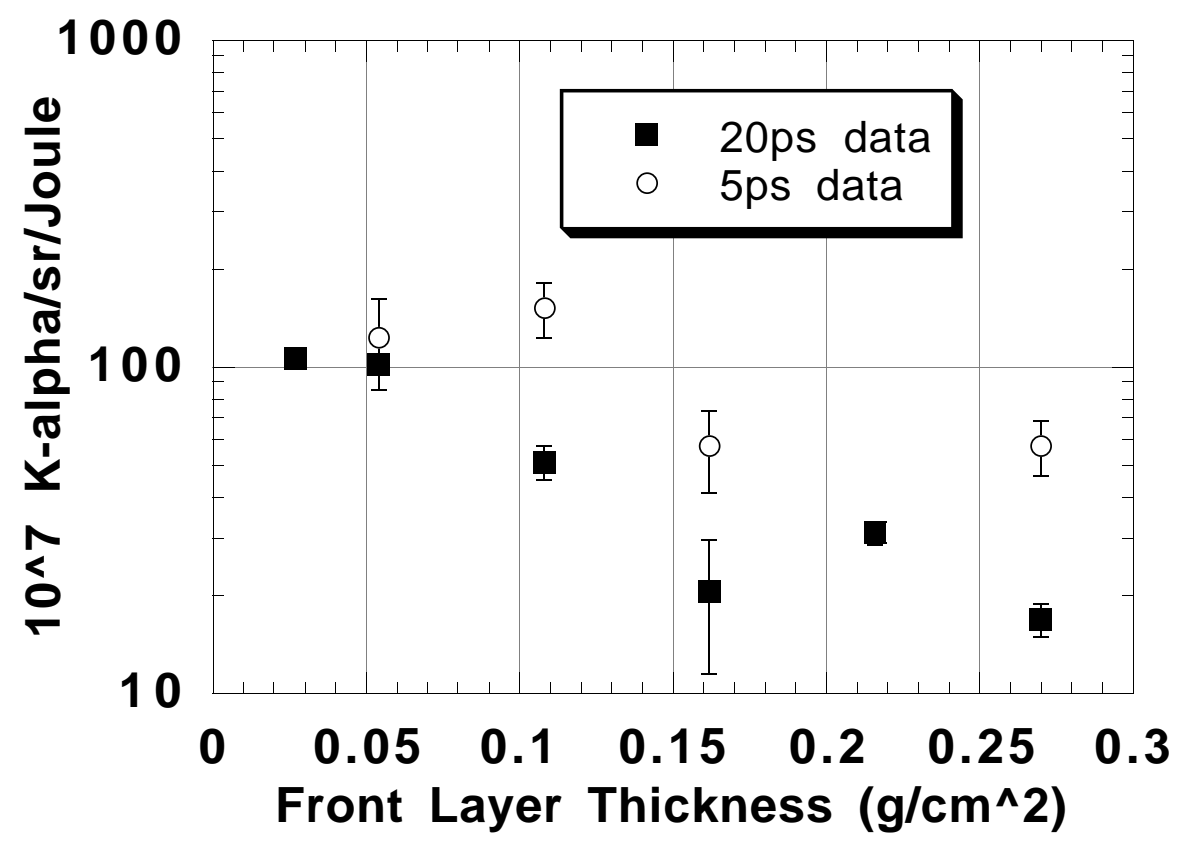

Figure 5.4. $K_{\alpha}$ signal from the $\mathrm{Al}$ targets, in units of $10^{7} \mathrm{x}$-rays per incident Joule and per steradian, plotted against areal mass of the Aluminum front layer of the target. The solid squares are experimental data at 20ps pulse length, empty circles are data at 5ps. ITS fits to this data were not possible because of the variation in laser intensity from shot to shot.

average energy $E_{0}=330 \mathrm{keV}$, which corresponds to a temperature of only $T_{\text {hot }}=170 \mathrm{keV}$. Therefore some new, high intensity mechanism may be limiting the average energy of electrons above $10^{18} \mathrm{~W} \mathrm{~cm}^{-2}$. If this new mechanism continued to limit $\mathrm{E}_{\mathrm{O}}$ up to intensities of $410^{19} \mathrm{~W} \mathrm{~cm}^{-2}$, then a constant $\mathrm{E}_{\mathrm{O}}$ for a wide range of intensities might be expected. This hypothesis does not contradict any previous experimental data, apart from measurements of the highest-energy electrons that do seem to scale as Eqn 2.8 predicts [54], and is consistent with all measurements presented thus far.

Once this assumption is made, every individual experimental $K_{\alpha}$ yield can be fit to $\mathrm{E}_{\mathrm{O}}=330 \mathrm{keV}$ spectrum, and therefore large data sets are no longer required. All ten 
data points from the Petawatt experiments (as well as all the 100TW data points) can each yield a conversion efficiency, assuming a 330keV average electron energy (and Maxwellian spectrum) in each case. This is calculated simply by comparing the data signal (normalized to the laser energy) to the ITS prediction for a $330 \mathrm{keV}$ electron spectrum propagated into that particular target. From those two numbers, one can determining the conversion efficiency required to produce the observed data. While this technique relies on an additional assumption not made in the 100TW analysis $\left(E_{0}=330 \mathrm{keV}\right)$, it seems the best way to draw conclusions from the limited data set.

Figure 5.5 shows the inferred conversion efficiencies plotted against laser intensity, and demonstrates a striking relationship between the two. Although this comparison is only as valid as the assumption of constant $\mathrm{E}_{\mathrm{O}}$, the result is a conversion efficiency that is highly dependent on the focal intensity of the laser. Further analysis of this graph will follow in the next section.

\subsection{Analysis}

The most obvious feature of Figure 5.5 is the upward trend of conversion efficiency with incident laser intensity; a straight line can almost be drawn through the data on a log-log plot. Although the data in Figure 5.4 was scattered and not very meaningful, the data from the very same shots appear nicely in Figure 5.5. While an obvious limit must be reached before the efficiency reaches $100 \%$, the saturation intensity has apparently not yet been achieved in these experiments.

This figure also links the Petawatt data to that of the 100TW laser at intensities near $10^{19} \mathrm{~W} \mathrm{~cm}^{-2}$. At this point the intensities of the two experiments come close to overlapping, and so do the conversion efficiencies. Although the energies, pulse length, and spot size are very different between the 100TW and the PW systems, it is encouraging to see a connection between the different laser experiments in Figure 5.5. 


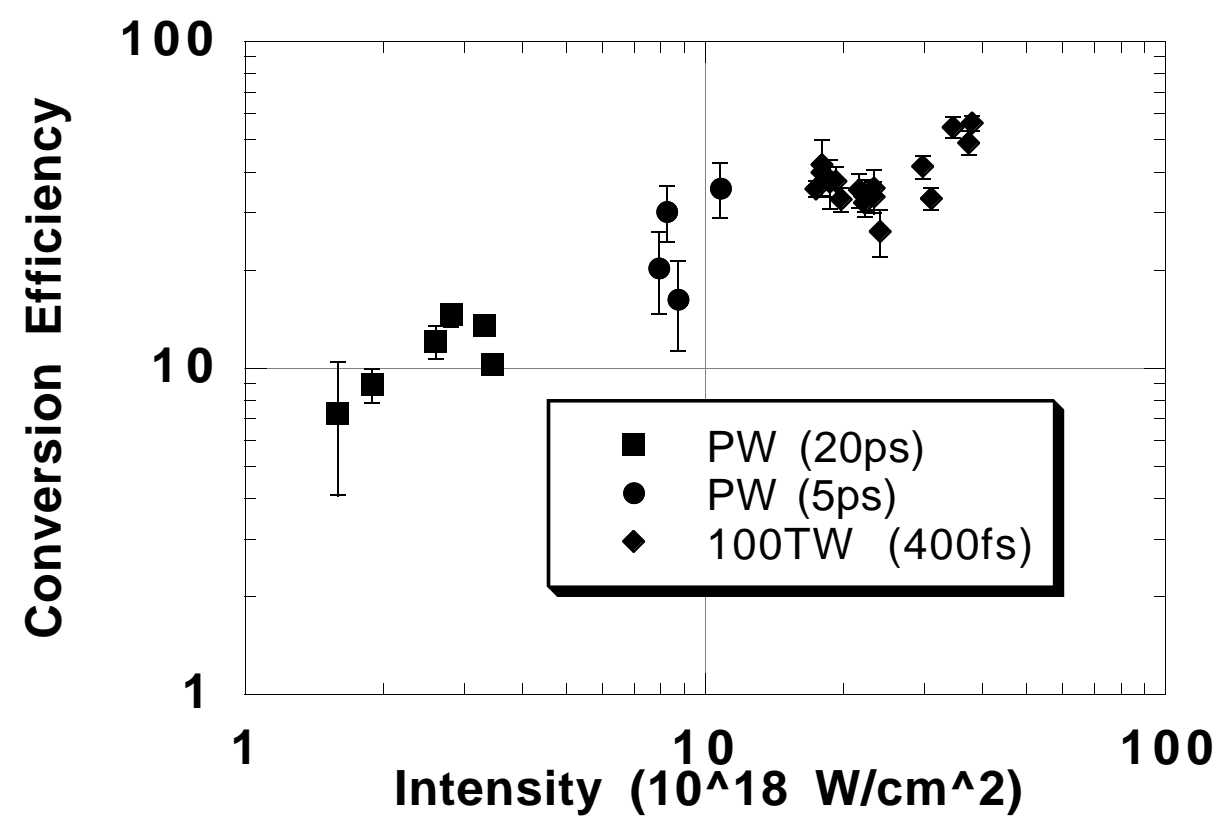

Figure 5.5. Laser-to-electron conversion effienciency is plotted against laser intensity assuming a $E_{0}=330 \mathrm{keV}$ electron spectrum for each shot. Squares are 20ps Petwatt shots, circles are 5ps Petawatt shots, and diamonds are 400fs 100TW shots. All efficiencies are multiplied by 0.7 if the electrons are assumed to be directed in a $30^{\circ}$ half-angle cone.

However, one could argue that the data in Figure 5.5 is scaling not with intensity, but with pulse length. The low intensity 20ps shots have the lowest conversion efficiency, the medium intensity 5 ps shots have a greater conversion efficiency, and the 400fs shots from the previous chapter are still the most efficient. Such an issue could be resolved by comparing short-pulse (400fs) PW shots with the previous $100 \mathrm{TW}$ data, but these experiments have not yet been carried out.

While a set of ten data points from experiments with varying laser parameters cannot be expected to reveal much physics, these preliminary indications should be able to guide future research on very high-power laser matter interactions. Already 
experiments are underway to maximize the x-ray flux produced by the Petawatt-produced fast electrons, for potential use in radiography experiments. Although trial-and-error techniques are possible, ideally one could maximize the x-ray flux with a deeper understanding of how the electrons are produced and transported in solid density targets. Issues of laser pre-pulse, laser focusing, and choice of target material present a large experimental phase space; basic research into these issues of hot electron production will continue to be an important field of study. Finally, the question of how these results (and those in the previous chapter) relate to an eventual fast ignitor fusion application will be discussed in Chapter 6. 


\section{Chapter 6}

\section{Implications for Inertial Confinement Fusion}

\subsection{Cross-Beam Effects}

\subsubsection{Scaling to NIF}

Although the motivation for the experiment presented in Chapter 3 was the crossed-beam geometry in NIF, there are nevertheless many differences between the exploding foil Nova experiment and an ignition-scale hohlraum. Therefore, despite the earlier positive results it is not a necessary conclusion that there will be any energy exchange between NIF beams, and the question becomes one of how the resonant instability might scale with the various changing parameters.

The linear theory, while inadequate for absolute levels of energy transfer, might still be relevant for scaling the parameters. Equation [2.6] is written here again for convenience:

$$
Q_{\max }=\frac{\pi}{2} \frac{n}{n_{c r}}\left(\frac{v_{o s c}^{2}}{v_{\text {the }}^{2}}\right) \frac{k_{i a} c_{s}}{\operatorname{Im}\left(\omega_{i a}\right)} \frac{L}{\lambda_{0}} \frac{1}{\left(1+3 T_{i} / Z T_{e}\right) \cos \theta_{s}}
$$

This equation shows the gain exponent $\mathrm{Q}$ is inversely dependent on $\operatorname{Im}\left(\omega_{\mathrm{ia}}\right) / \operatorname{Re}\left(\omega_{\mathrm{ia}}\right)$ (in the plasma frame); ion Landau damping prevents large ion waves and therefore limits energy transfer. Recall Eqn. [1.11] expressed $-\operatorname{Im}\left(\omega_{\mathrm{ia}}\right) / \operatorname{Re}\left(\omega_{\mathrm{ia}}\right)$ as a function of $\theta=Z \mathrm{~T}_{\mathrm{e}} / \mathrm{T}_{\mathrm{i}}$, maximum for a value of $\theta=2.45$.

In NIF, the gas in the hohlraum (used to prevent expansion of the gold walls) will likely be a mixture of $\mathrm{H}$ and $\mathrm{He}$. Gold from the walls will also be present, but the very 
high $\mathrm{Z}$ of the gold makes its contribution to ion Landau damping negligible (although it may be important in other ways). With expected values of $\mathrm{ZT}_{\mathrm{e}} / \mathrm{T}_{\mathrm{i}}=\sim 6$, ion Landau damping in NIF will likely be somewhat larger than in our exploding foil experiments.

The exponential gain $\mathrm{Q}$ is also proportional to the density $\left(\mathrm{n} / \mathrm{n}_{\mathrm{cr}}\right)$ and the laser intensity ( $\left.\mathrm{I} \alpha \mathrm{v}_{\mathrm{Osc}}{ }^{2}\right)$ and is inversely proportional to the electron temperature $\left(\mathrm{T}_{\mathrm{e}} \alpha \mathrm{v}_{\mathrm{the}}{ }^{2}\right)$. A rough estimate of these parameters in NIF can be given by LASNEX simulations [69]. The following table summarizes the change in these parameters between NIF (at the time of peak laser intensity) and the exploding foil Nova experiment from Chapter 3:

\begin{tabular}{lll} 
& \multicolumn{1}{l}{ Nova (foil) } & NIF \\
$\mathrm{Z}$ & 4 & 1.5 \\
$\mathrm{~T}_{\mathrm{e}}(\mathrm{keV})$ & 1 & 4 \\
$\mathrm{ZT}_{\mathrm{e}} / \mathrm{T}_{\mathrm{i}}$ & $\sim 8$ & $\sim 6$ \\
$\mathrm{I}\left(\mathrm{W} \mathrm{cm}{ }^{2}\right)$ & $10^{15}$ (pump) & $2.410^{15}$ \\
$\mathrm{n} / \mathrm{n}_{\mathrm{cr}}$ & 0.06 & 0.015 \\
$\operatorname{Im}(\omega) / \operatorname{Re}(\omega)$ & .11 & .20
\end{tabular}

Table 6.1. Various plasma parameters contrasted between NIF and the exploding foil experiment performed on Nova (from Chapter 3 ).

Because the pump and probe intensities were different in the NOVA experiment, $\mathrm{v}_{\mathrm{Osc}}{ }^{2}$ is proportional to the geometric mean of the two beam intensities, lowering this parameter by the square root of the intensity ratio of the two beams. Leaving aside the gain length parameter L (which, as discussed in chapter 3, can be artificially shortened by many different effects), the $\mathrm{Q}$ in the Nova experiment turns out to be several times of that predicted for NIF. 
Given the large uncertainty in the theory, however, it would be more prudent to assume that the resonant ion waves in NIF will reach the same amplitude and transfer the same amount of power ( 100J per ns) between beams as in the exploding foil experiment. Because the average power of a 4 -beam cluster is $\sim 4 \mathrm{~kJ}$ per $\mathrm{ns}$, this would only be a $2.5 \%$ perturbation. But the net effect, taking into account the loss from the inner cones into the outer cones, would be on the $5 \%$ level. This is smaller than the $8 \%$ rms balance in beam intensity required for ignition, but it would not be randomly distributed; all the laser energy would move from the inner- to the outer-cone. Adding in the expected fluctuations between the laser intensities might lead to a significant asymmetry in the fuel compression. This effect could conceivably be much larger if the mitigating effects that limited the energy transfer small in the exploding foil did not apply in NIF.

However, many mitigating effects could be larger in NIF, such as the filamentation and non-Maxwellian electron distributions (as discussed in chapter 3). Because Eqn. [2.6] was not accurate in predicting the amount of energy transfer, these scaling arguments can only be considered a rough and inaccurate estimate, and no firm conclusions of crossed-beam effects in NIF can be made at this point.

It should be noted, however, that the LASNEX simulation of energy transfer assumes that none of the 192 beams actually hits the side of the hohlraum. NOVA experiments have shown that this is not an accurate assumption, and that the plasma density outside the hohlraum is larger for this reason. The blow-off of the gold material (and surrounding Be shell) would not only raise the density, but also change the other plasma parameters $\left(\mathrm{Z}, \mathrm{T}_{\mathrm{e}}, \mathrm{T}_{\mathrm{i}}\right)$ that could drastically change all of the above scaling arguments. Further importance of this additional plasma will be discussed in the next section. 


\subsubsection{Resonance and k-matching in NIF}

Apart from the damping mechanisms, the critical parameter for resonance is the flow velocity of the plasma. If a Mach 1 flow is not reached (in the proper direction), there should be no resonant instability. The possible resonances are numerous; the 192 $\mathrm{f} / 20$ beams of NIF are arranged into $48 \mathrm{f} / 8$ 4-beam clusters on each side of the hohlraum, and each cluster can typically be treated as a single beam. Because all 48 beams pass through the center of the hohlraum's laser entrance hole (LEH), there are (in principle) over a thousand different crossed-beam interactions on each side!

Cylindrical geometry is necessary to discus the geometry of NIF; the z-axis is defined as the hohlraum axis, and the LEH therefore lies in a radial plane. On one end of the hohlraum, the 48 beams come in at 4 cone angles with respect to the z-axis: $23.5^{\circ}$, $30^{\circ}, 46.5^{\circ}$, and $50^{\circ}$. Each beam is $\mathrm{f} / 8$, giving them all $\pm 3.5^{\circ}$ of angular beam width. Because of this angular spread, the four beam cones can also be viewed as two extended cones, the inner cone extending from $20^{\circ}$ to $33.5^{\circ}$, and the outer cone extending from $43^{\circ}$ to $53.5^{\circ}$. Because of the beam-width and the large number of beams, a decent approximation is that laser light approaches the hohlraum from all $2 \pi$ azimuthal angles.

The most important interactions will be those where the matching ion wave kvector $\left(\mathbf{k}_{\mathbf{i a}}\right)$ is closest aligned with the plasma flow $\left(\mathbf{v}_{\mathbf{f}}\right)$ out of the hohlraum; those interactions will maximize the $\mathbf{v}_{\mathbf{f}} \mathbf{k}$ product in Eqn. [1.10] and come closest to shifting the ion wave frequency to zero in the laboratory frame. Therefore the important interactions are the ones from laser beams in the same azimuthal direction. The other crossed-beam interactions cannot be completely ignored, but to first order they are not as important.

Looking at the geometry of any two beams with the same azimuthal angle, one can define $\alpha$ and $\beta$ as the angles the beams make with the z-axis. The bisector angle $(\alpha+\beta) / 2$ is perpendicular to the matching $\mathrm{k}$-vector of the ion wave at the other end of the 
triangle, and therefore the angle $\gamma$ between $\mathbf{k}_{\mathbf{i a}}$ and $\mathbf{z}$ is $\gamma=(\pi-\alpha-\beta) / 2$. The necessary Mach number (along $\mathbf{z}$ ) to achieve a Mach 1 flow along $\mathbf{k}_{\mathbf{i a}}$ can then be calculated as $\sec (\gamma)$. For smaller $\gamma$, less plasma flow is needed to reach resonance, and therefore largeangle beams (large $\alpha, \beta)$ are the most likely to reach resonance.

Following this logic, the largest angle in each of the two extended beam cones occur at $\alpha=33.5^{\circ}$ and $\beta=53.5^{\circ}$, yielding the value $\gamma=46.5^{\circ}$. This corresponds to a $z-$ directed plasma flow of Mach 1.45 necessary to achieve exact resonance. Smaller plasma flows can achieve resonance if one takes into account the resonance half-width $\operatorname{Im}\left(\omega_{\mathrm{ia}}\right) / 2$ (see section 2.2.2). In the NIF plasma, the ratio $\operatorname{Im}\left(\omega_{\mathrm{ia}}\right) / \operatorname{Re}\left(\omega_{\mathrm{ia}}\right)$ is expected to be 0.2 , so this resonance width will ease the flow velocity requirement by $\pm 10 \%$. Further relaxation of the flow velocity limit would arise from any bandwidth on the incident beams; additional frequency components would allow lower-frequency ion waves, which would require accordingly less plasma flow to reach resonance. Detailed simulations of this scenario have been performed $[69,70]$ which indicate a possible resonance at the outer extent of the beam crossing region even without extra bandwidth on the lasers.

Energy transfer between the inner- and outer- beam cones is of primary concern because the pulse shapes and intensities are different in the two cases, and any mixing of the two shapes could cause a dramatic decrease in hohlraum symmetry. However, there is another possibility; that of the different angular components of the outer beam cone interacting with itself. If an outer-cone beam at $\alpha=43^{\circ}$ crosses another outer-cone beam at $\beta=53.5^{\circ}$, the larger beam angles now reduce $\gamma$ to $41.75^{\circ}$. This corresponds to a Mach 1.34 flow, pushing the resonant flow velocities even lower than in the previous case.

While the symmetry would not be as strongly affected in this case (all outer-cone beams have the same pulse-shape), the carefully balanced NIF hohlraum might still be adversely affected by such a resonance. An important difference in this case, however, 
would be the very small value of $\mathbf{k}_{\mathbf{i a}}$ caused by the nearly-parallel incident beams. Such a small k-vector would correspond to a long wavelength ion wave, and therefore any inhomogenieties in the plasma would become that much more important. Spatial perturbations smaller than $\mathbf{k}_{\mathbf{i a}}{ }^{-1}$ might even prevent any resonant energy transfer altogether.

An even more dangerous situation could arise if any of the incoming beams clip the gold wall. This possibility was mentioned at the end of section 6.1 .1 and would only require a small mispointing error on a few of NIF's 192 beams. The resulting blow-off plasma would completely change all of the above analysis, and would be more conducive to reaching an ion-wave resonance.

Geometrically, the assumption has been that the main plasma flow will be directed along the z-axis. This would no longer be true if the hohlraum was clipped by the laser beams; now plasma would be exploding off of the LEH in all directions. Plasma that left the LEH walls at a $45^{\circ}$ angle would intersect the beam-crossing region on the zaxis, and now the flow could be exactly oriented in the same direction as $\mathbf{k}_{\mathbf{i a}}$. This would mean that only a Mach 1.0 flow would be needed to reach resonance.

Further dangers exist because the sound speed in this colder, higher-ion mass plasma will be much lower. Recall from Eqn. 1.9 that $\mathrm{c}_{\mathrm{s}} \alpha \mathrm{ZT} \mathrm{T}_{\mathrm{e}} / \mathrm{M}$. Although the outside of the hohlraum will likely be coated in low-Z Be, gold will inevitably escape as well. Even with a charge state of 40, Z/M for gold would be three times less than that for the $\mathrm{H}$-He plasma. $\mathrm{T}_{\mathrm{e}}$ would also be lower (both for $\mathrm{Be}$ and $\mathrm{Au}$ ) because the plasma would not be originating from inside the hohlraum. The much smaller sound speed would mean that lower absolute velocities would be necessary to achieve resonance.

Possible experimental verification of this effect has recently occurred in a Nova hohlraum experiment [71]. Two halves of a single Nova beam were independently 
modified and then brought together in the LEH of an empty NOVA hohlraum. Although no mispointing errors were discovered, the centroid of the beam on the inner hohlraum wall was discovered to be significantly shifted away from the hohlraum center. This would be consistent with the above analysis if various NOVA beams clipped the LEH and created a flowing $\mathrm{Au}($ and $\mathrm{Be}$ ) plasma in which the two halves of the Nova beams intersected. The inner-half of the beam (directed closer to the center of the hohlraum) might have resonantly transferred energy to the outer-half, shifting the centroid of the beam energy to a steeper incident angle. While this preliminary result is certainly not proof of a crossed-beam effect in a hohlraum, it is cause for concern.

Because there are many differences from the NOVA experiment, it is impossible to determine (at this point) if resonant crossed-beam energy transfer will be relevant in ignition-scale hohlraums. The above analysis, however, suggests that resonance conditions are likely to be present in the current NIF design. Possible methods to avoid such a resonance will be explored in the following section.

\subsubsection{Possible Solutions}

If the high Landau damping of NIF is not enough to prevent dangerous levels of energy transfer between beams, the only obvious solution is to prevent the resonance from occurring in the first place. Although the beam geometry is fixed, as is the location of the crossing-beam region, there is still one key parameter that is available: the relative frequency of the beam cones.

The original plans for NIF included 4-color operation, or separating the frequencies of the beamlets in each four-beam cluster by a small amount. Concern was then raised of the heightened potential for resonant instabilities, as the frequency differences between the laser beams were more likely to seed ion-wave resonances such as SBS. Therefore 4-color operation is not planned for NIF at this time. However, the 
capability for frequency-modification will be retained for the four NIF beam cones, which would allow a 2- or 4-color system where the different colors would be on the different cones rather than in a single 4-beam cluster.

Such a capability would obviously be useful for detuning a resonant instability between the NIF beam cones. Any two beams of comparable frequency will be resonant with a third ion wave at exactly two plasma velocities; one velocity for each direction of energy transfer. For two identical frequency beams, the analysis in Chapter 2 showed that these two velocities were $\pm 1.0 \mathrm{c}_{\mathrm{s}}$ (along the k-matching axis). Previous experiments [29] have shifted the frequency of one beam by exactly $\omega_{\text {ia }}$, matching to a resonance in a stationary plasma (and also allowing another resonance at Mach 2.0). Ideally in NIF one could upshift the outer-cone beams by $\sim 0.8 \omega_{\mathrm{ia}}$. This would allow resonant energy transfer from the outer- to the inner- beams at a Mach -0.2 flow into the hohlraum (very unlikely), and would raise the necessary outward flow for energy transfer in the other direction to a minimum of Mach 1.8. Therefore by shifting the frequencies, the resonance at Mach 1 might be completely detuned in NIF.

Another solution, possible if the plasma flow in the LEH is reproducible from shot to shot, is so simply accept that energy will be transferred and to weight the NIF beams accordingly. This might be feasible because little energy is transferred to the resonant ion wave itself; most of the energy will remain as laser light. Such a solution would require very reproducible laser and a time-intensive scanning of parameter space that must be done experimentally (simulations are unlikely to accurately predict plasma flow out of the LEH). However, because the inner-and outer- beam cones of NIF have different pulse shapes, this would primarily be a mechanism to counter energy exchange in the same beam cone, not between beam cones. 


\subsubsection{Future Experimental Work}

Many important issues concerning resonant crossed-beam effects can be further explored with existing lasers such as NOVA and OMEGA. Such work, done before NIF is built, could save valuable time if such effects are determined to be important.

One obvious extension is to do this experiment in an actual hohlraum, rather than in an exploding foil plasma. This option was considered and rejected because of the diagnostic difficulties created by the presence of the hohlraum itself. However, if one did not measure the transmitted laser energy (as was done in Chapter 3) but rather measured the x-ray yield from the hohlraum walls, one could determine where the laser energy was deposited. This technique could (in principle) allow a rough measurement of energy transfer in a hohlraum geometry. In addition, these shots might be possible as "ride alongs" on other hohlraum experiments on Nova or Omega, providing the data without the expense of a dedicated laser shot.

Further scaling experiments would be useful as well. An experiment in lower-Z $\mathrm{H}$ or He plasma (as opposed to $\mathrm{Be}$ ) would be more relevant for NIF. Experiments in $\mathrm{Au}$ plasma could also determine the importance of beam-clipping on the LEH. More detailed scaling of pump/probe intensity ratios would be informative, especially if the pump intensity was varied in addition to the probe. A "forward-scattering" crossed beam geometry, with the beams propagating in almost the same direction, would also be an important extension of this work.

While many unknowns remain concerning resonant energy transfer between two identical-frequency beams in a flowing plasma, the experiment presented in this thesis has determined for the first time that it is an observable effect with potentially serious consequences. Although final determination of this effect's importance may require the completion of NIF, the experiment presented here has already helped ensure the retention 
of the "2-color" option in NIF's final design. Further experimental work along these lines will likely be of equal importance.

\subsection{Fast Ignition}

\subsubsection{General Implications}

Scaling difficulties even more dramatic than those in section 6.1 apply when attempting to draw conclusions on the feasibility of the fast ignitor concept based on the experiments presented in Chapters 4 and 5. The most obvious differences between these experiments and an actual fast-ignitor experiment is the cold, solid density target. In a fusion-yielding interaction, a fuel pellet (DT) would already have been significantly compressed and heated. An underdense plasma would also exist around the pellet, more extensive than the plasma formed by the prepulse of the lasers described in this thesis.

Given all these differences, only limited conclusions can be drawn from the experiments in this thesis. The first, and most important, result is that extremely large currents of electrons can propagate into solid-density material. Had there been some fundamental physical mechanism that prevented this, the entire fast ignitor scheme would have been found highly questionable.

The magnitude of the current measured in our experiments is easily determined from the conversion efficiency $(\eta)$, the laser energy $\left(E_{\text {laser }}\right)$, the average electron energy $\left(\mathrm{E}_{0}\right)$, and the pulse length ( $\left.\tau_{\text {laser }}\right)$. The current density is less easily calculated (the electron beam diameter is unknown), but all of the basic limits described in Chapter 4 apply to net current (i.e. the Alfven current). As current is merely charge per unit time, the equation for current appears as:

$$
I=\frac{\eta e E_{\text {laser }}}{E_{0} \tau_{\text {laser }}}=\frac{10^{6} \eta E_{\text {laser }}(J)}{E_{0}(M e V) \tau_{\text {laser }}(p s)}
$$


Note Eqn. 6.1 is in MKS units, so that the current comes out in Amperes. For the highest intensity shots on Aluminum in Chapter 3, this corresponds to a current of 68MA. Comparing the Alfven current for $330 \mathrm{KeV}$ electrons (mildly relativistic), Eqn. 4.3 yields $I_{A}=22 k A$. The number of Alfven currents we measure is therefore $N_{A} \approx 3000$.

Comparing to the back-of-the-envelope calculation for the current limit in Eqn. 4.5 , one can calculate that fully ionized $(Z=13)$ solid aluminum should have an electron density of $810^{23} \mathrm{~cm}^{-3}$. Plugging this in as the density n, and assuming that the electron beam radius corresponds to the laser spot size (10 laser wavelengths), one gets $\mathrm{N}_{\mathrm{A}} \approx 1000$. Although we seem to measure a larger current than this supposed maximum, slight modification of the parameters (50\% larger spot size and 50\% longer electron pulse) can easily bring the two numbers in line. Indeed, one would expect both; the electron beam will likely widen as it propagates. Also, the FWHM of the pulse is $0.4 \mathrm{ps}$, which was used for the parameter $\tau_{\text {laser }}$ in Eqn. 6.1; however a sizable amount of energy is deposited outside this time period.

An important point is that although the ultra-high current electron beam was "created" in an underdense plasma where the laser could penetrate $\left(\mathrm{n}=10^{21} \mathrm{~cm}^{-3}\right)$, the electrons were still able to propagate into the solid-density region despite the smaller limiting current in the underdense region $\left(\mathrm{N}_{\mathrm{A}} \approx 20\right)$. This is a crucial fact for the fast ignitor concept; was this not possible, the scheme could never work.

Also, the experiments presented here show that the actual current limit cannot be much smaller than Eqn. [4.5] signifies. But although our results represent some of the highest measured currents to date, we cannot conclude if currents larger than those predicted by Eqn. [4.5] are possible.

If Eqn. [4.5] is correct, what implications exist for the fast ignitor? Ideally, one would spark a fusion reaction with $10 \mathrm{~kJ}$ of $1.0 \mathrm{MeV}$ electrons in a $10 \mathrm{ps}$ pulse; this corresponds to 1 GigaAmp in Eqn. [6.1]. This is only 15 times larger than measured 
currents in the experiments, and is even closer to the number of Alfven currents: $1 \mathrm{MeV}$ electrons correspond to $I_{A}=47 \mathrm{kA}$, so for the fast ignitor $\mathrm{N}_{\mathrm{A}}=21,000$ : seven times larger than already measured. A seven-fold increase in $\mathrm{N}_{\mathrm{A}}$ would be allowable under Eqn. [4.5] if a DT fuel pellet was compressed to $160 \mathrm{~g} / \mathrm{cm}^{2}$. This is a reasonable requirement (such densities are required for ignition regardless), and therefore there appears to be no fundamental reason why such an electron beam could not be propagated into a compressed pellet. The distance between the underdense region and the compressed fuel region, however, must be kept as short as possible so that the electrons can propagate into the dense fuel before their self-generated magnetic fields can pinch off the current.

As mentioned at the end of Section 4.1.3, multiple seperate filaments of electrons can also explain the large number of Alfven currents. At the plasma density of fully stripped solid Aluminum, the magnetic skin depth is only $0.006 \mu \mathrm{m}$, much smaller than the $15 \mu \mathrm{m}$ laser beam diameter. Even if each filament of electrons had a diameter equal to 10 magnetic skin depths, over 60,000 filaments could fit inside the two-dimensional beam profile (at solid density). Given that each filament could hold an Alfven current, this could easily explain the large currents seen in the experiments. Again, this scenario would allow sufficient currents for the fast ignitor scheme.

Further conclusions can be drawn from the experiments presented in Chapters 4 and 5. Large ( $30 \%)$ conversion efficiencies seem to be possible at high intensities (but modest energies). These conversion efficiencies will be crucial for any eventual fast ignitor application; if the fraction of energy converted to electrons was less than 5\%, tremendously large (>200kJ) ignitor beams would be necessary.

The electron energy dependence on target conductivity is an interesting and new result at these intensities, and it points to the data in copper targets as more relevant for the fast ignitor. This is because hot DT plasma will have roughly the same conductivities as our $\mathrm{Cu}$ targets. Although the intensity will be greater for a fast-ignitor scenario, the 
apparent saturation of electron energy (as a function of laser intensity), might imply that the electron beam in a fast-ignitor scenario might be the $640 \mathrm{keV}$ energy measured in 100TW experiments. This would be lower than the desired $1 \mathrm{MeV}$ beam, but possibly large enough to still spark a fusion reaction. Further intensity scaling (to $10^{20} \mathrm{~W} \mathrm{~cm}^{-2}$ and beyond) would be crucial for determining the feasibility of this scheme.

\subsubsection{Future Work}

Even if the results of $\sim 30 \%$ conversion efficiency and near-MeV electron energies would continue hold for fast ignitor-relevant parameters, there are other obstacles on the road to a high-yield fusion reaction. The plasma blowoff from the solid targets (as discussed in Chapter 4) can be much greater in a true compression-ignition scheme, and the presence of this large underdense plasma might significantly alter the laser-plasma interaction. The channeling pulse, which would be necessary to get the ultra-highintensity laser anywhere near the compressed fuel, has not yet been discussed; if this is not feasible the fast ignitor scheme would most likely fail. Perhaps even the distance from the critical density (where the electrons are produced) to the region of $200 \mathrm{~g} / \mathrm{cm}^{3}$ compressed fuel (where the huge return current must come from) might prove to be the crucial parameter in a fast ignition reactor.

In order to address these and other issues, experiments are continuing on the Petawatt laser and are also being planned on other high-energy, short-pulse laser systems currently in construction around the world. Based on the above analysis of the electron return current, many fast-ignitor-relevant experiments cannot be performed at solid density; there would not be a sufficiently dense background to propagate the necessary currents. Instead, the ability to compress targets with additional laser beams will be necessary to truly test the physics of the fast ignitor scheme. Tentative plans are being developed at Lawrence Livermore Lab to interface the Petawatt with one Nova beam, 
allowing planar compression of small targets. Experiments requiring spherical compression may have to await the construction of a short-pulse system at a large laser facility (NIF, Omega, etc.).

Based on the results presented in this thesis, some important avenues of future research include:

1) Continuation of the electron measurements to intensities above $10^{20} \mathrm{~W} / \mathrm{cm}^{2}$.

2) Scaling of conversion efficiency in pre-compressed targets.

3) More accurate electron beam cone-angle measurements, including magnetic field diagnostics.

4) Theoretical understanding of the relationship between laser intensity and electron conversion efficiency.

5) Effect of the laser-prepulse and associated pre-plasma.

Ideally the cost and size of high-intensity lasers will continue their exponential decrease, while the shot rate will go up dramatically. If such laser advances continue, some of these research topics may be addressed outside of the world's largest laser systems. Right now the limiting factor in these large systems is damage to the compression gratings; the PW gratings must be meter-sized as a result. Perhaps one additional area of important laser-plasma research might be an attempt to make a compression grating out of a plasma, similar to a "plasma mirror". Such a damage-free grating could allow table-top Petawatt laser systems which would in turn allow laboratories everywhere to perform experiments relevant to fast ignitor fusion.

\subsection{Summary}

Inertially confined fusion may be another century away, or perhaps a series of breakthroughs will allow a fusion power plant to be constructed early in the next century. New ideas such as the fast ignitor concept will be crucial to any eventual success. 
While the experiments presented in this dissertation may or may not help guide the way to a future fusion reactor, it is notable that they did not turn up any "showstoppers", either for fast ignition or for conventional ICF. The experiments presented in Chapter 3 might have determined that the $100 \%$ energy transfer between crossed-beams (as predicted by linear theory) actually does occur, rather than the low levels of absolute energy transfer seen in the experiment. The experiments presented in Chapter 4 and 5 might have discovered numerous flaws in the fast ignition scheme, such as low conversion efficiencies, too-high electron energies, or an inability of targets to support many Alfven currents.

Apart from fusion considerations, the experiments presented in Chapter 3 have shown for the first time that energy can be resonantly transferred between two beams of equal frequency in a flowing plasma, and that this energy transfer is lower than predicted by linear theory. The scaling of this effect suggests that the interaction is only taking place over a small fraction of the crossing beams.

The experiments on the 100TW laser have shown that 30\% laser-to-electron conversion efficiency is possible in ultra-high intensity laser-matter interactions. These measurements of conversion efficiency were the first to be done in this intensity regime. Additional observations included an intriguing dependence on target material, an apparent beaming of the highest energy electrons, and a curious independence of electron energy on laser intensity.

Continuing these experiments on the Petawatt laser, it was discovered that very energetic laser pulses up to $400 \mathrm{~J}$ can still have sizable conversion efficiency, despite the larger number of electrons needed to carry the current. In addition, a straightforward relationship between laser intensity and conversion efficiency was discovered over a large range of high intensity interactions. Similar intensities to the 100TW are not yet 
available at these energies, but experiments of this nature will no doubt continue into the future. 


\section{Appendix A}

\section{Energy Transfer in a General Three-Wave Resonance}

\section{A.1 Mathematical Model}

For three resonant waves all propagating in the x-dimension, the wave amplitudes can be written:

$$
\begin{aligned}
& a_{1}(x, t) e^{i k_{1} x-i \omega_{1} t}+c . c . \\
& a_{2}(x, t) e^{i k_{2} x-i \omega_{2} t}+c . c . \\
& a_{3}(x, t) e^{i k_{3} x-i \omega_{3} t}+c . c .
\end{aligned}
$$

Resonance occurs if the usual matching conditions hold:

$$
k_{1}+k_{2}=k_{3} ; \omega_{1}+\omega_{2}=\omega_{3}
$$

Each wave $a_{n}$ is a normal mode of the plasma, so that (as discussed in Chapter 1) it can be represented by the usual damped oscillator equation in the absence of other waves or fields:

$$
\begin{aligned}
& \mathrm{D}_{\mathrm{n}}(\omega, k)\left[a_{n} e^{i k_{n} x-i \omega_{n} t}+c . c .\right]=0 \\
& \mathrm{D}_{\mathrm{n}}(\omega, k)=-\omega^{2}-2 i \Gamma_{n}(k) \omega+\omega_{n}^{2}(k)+\Gamma_{n}^{2}(k)
\end{aligned}
$$

At this point the "slowly varying envelope approximation" must be made, which is that the wave amplitudes $a_{n}(x, t)$ vary slowly as compared to the regular exp(ikx-i $\left.\omega t\right)$ oscillation. Removing the oscillatory component, this approximation can be written as:

$$
\mathrm{D}_{\mathrm{n}}\left(\omega_{n}+i \frac{\partial}{\partial t}, k_{n}-i \frac{\partial}{\partial x}\right)\left[a_{n}\right]=0
$$


Expanding [A.5] to first order in $\delta / \delta$ t and $\delta / \delta x$, and neglecting $\Gamma^{2}$;

$$
-2 i \omega_{n}\left[\Gamma_{n}+\frac{\partial}{\partial t}+\frac{\partial \omega_{n}}{\partial k} \frac{\partial}{\partial x}\right] a_{n}=0
$$

Here $\delta \omega_{\mathrm{n}} / \delta \mathrm{k}=\mathrm{V}_{\mathrm{n}}$ is the group velocity of the wave, and the wave is still undriven by other fields. Adding coupling between the waves requires that the zero on the right side of Eqn. [A.6] be replaced by the amplitudes of the other waves as well as a coupling constant which is determined by the strength of the coupling between these particular waves. (The fields produced by other interactions will not satisfy Eqn [A.2] and are therefore off-resonant and need not be considered.) Writing all three coupled equations then takes the form [72]:

$$
\begin{aligned}
& \frac{\partial a_{1}}{\partial t}+\Gamma_{1} a_{1}+V_{1} \frac{\partial a_{1}}{\partial x}=\beta a_{2}^{*} a_{3}^{*} \\
& \frac{\partial a_{2}}{\partial t}+\Gamma_{2} a_{2}+V_{2} \frac{\partial a_{2}}{\partial x}=\beta a_{1}^{*} a_{3}^{*} \\
& \frac{\partial a_{3}}{\partial t}+\Gamma_{3} a_{3}+V_{3} \frac{\partial a_{3}}{\partial x}=-\beta a_{1}^{*} a_{2}^{*}
\end{aligned}
$$

The coupling constant $\beta$ comes with a negative sign in the final equation because it represents the highest-frequency wave $\left(a_{3}\right)$ and from Eqn [A.2] $\omega_{3}$ is the sum (rather than the difference) of the other two frequencies.

If both damping and the convective term $(\mathrm{V} \delta \mathrm{a} / \delta \mathrm{x})$ are neglected, the following parameters can be shown to be constant by performing a time derivative and comparing to [A.7]:

$$
\begin{aligned}
& \left|a_{1}\right|^{2}+\left|a_{3}\right|^{2} \\
& \left|a_{2}\right|^{2}+\left|a_{3}\right|^{2} \\
& \left|a_{1}\right|^{2}-\left|a_{2}\right|^{2}
\end{aligned}
$$

These constants represent conservation of action, also known as the Manley-Rowe relations. [73] An additional constant arises from total energy conservation, with the 
energy in a given wave proportional to the frequency (see the quantum analogy in Section 2.1.2):

$$
\omega_{1}\left|a_{1}\right|^{2}+\omega_{2}\left|a_{2}\right|^{2}+\omega_{3}\left|a_{3}\right|^{2}=\text { Constant }
$$

\section{A.2 Resonant Energy Transfer}

The three equations in [A.7] contain all the physics of three-wave resonant interactions, but are not analytically solvable. Removing the damping and the convective term does allow an analytical solution in terms of Jacobi elliptic functions [74]. Without damping, however, one does not generally find net (steady-state) energy transfer between the waves; eventually the energy will return. Therefore the no-damping case will not be discussed here.

The simplest form of [A.7] with damping is to assume that one of the three waves is heavily damped, while the other two have weak damping that can be neglected. This is often a relevant scenario, such as when two electromagnetic waves interact with a third plasma wave. If the damped wave is $\mathrm{a}_{1}$, and the damping dominates both the time derivative and the convective term, the first of [A.7] can be written:

$$
a_{1}=\frac{\beta a_{2}^{*} a_{3}^{*}}{\Gamma_{1}}
$$

Substituting [A.10] into the other two Equations in [A.7], and again ignoring the convective terms, one can easily find:

$$
\begin{aligned}
& \frac{\partial}{\partial t}\left|a_{2}\right|^{2}=2|\beta|^{2} \Gamma_{1}^{-1}\left|a_{2}\right|^{2}\left|a_{3}\right|^{2} \\
& \frac{\partial}{\partial t}\left|a_{3}\right|^{2}=-2|\beta|^{2} \Gamma_{1}^{-1}\left|a_{2}\right|^{2}\left|a_{3}\right|^{2}
\end{aligned}
$$

Neglecting the convective terms is not a good approximation for fast-moving electromagnetic waves, although these results are shown to be equivalent to the more relevant "steady-state approximation" in the following section. Defining the intensity of 
each wave $I_{n}=\left|a_{n}\right|^{2}$, the variables can be separated using the Manley-Rowe relation $I_{2}+I_{3}$ $=$ constant (second Eqn. of [A.8]). The solution then becomes:

$$
\begin{aligned}
& I_{2}(t)=\frac{I_{2}(0)^{2}+I_{2}(0) I_{3}(0)}{I_{3}(0) e^{-\Gamma t}+I_{2}(0)} \\
& I_{3}(t)=\frac{I_{3}(0)^{2}+I_{2}(0) I_{3}(0)}{I_{2}(0) e^{\Gamma t}+I_{3}(0)}
\end{aligned}
$$

where:

$$
\Gamma=\frac{2|\beta|^{2}\left(I_{2}(0)+I_{3}(0)\right)}{\Gamma_{1}}
$$

The relative energy gain of $\mathrm{I}_{2}$ is therefore:

$$
\frac{I_{2}(t)-I_{2}(0)}{I_{2}(0)}=\frac{\left(1-e^{-\Gamma t}\right)}{e^{-\Gamma t}+I_{2}(0) / I_{3}(0)}
$$

The result of these calculations is to show that energy will transfer from the higher-intensity undamped wave $\left(I_{3}\right)$ to the lower-intensity undamped wave $\left(I_{2}\right)$ because of the interaction with the damped wave $\mathrm{I}_{1}$. This is an irreversible process; the energy does not flow in the opposite direction at later times, as was the case in the undamped oscillators.

This direction of energy flow can also be illustrated with an entropy argument. The number of quantum (photons, phonons) in a laser or plasma wave can be found simply by dividing the intensity by the quantum energy $h \omega / 2 \pi$, defining the number of quantum to be $N_{1}=2 \pi I_{1} / h \omega$, etc. The entropy of the three-wave system is the log of the number of possible states. Given that photons and phonons are interchangable within a given wave, this entropy is therefore:

$$
S=\ln \left(\frac{\left(N_{1}+N_{2}+N_{3}\right) !}{N_{1} ! N_{2} ! N_{3} !}\right)
$$

Because entropy must increase over time, the total number of quantum $\left(\mathrm{N}_{1}+\mathrm{N}_{2}+\mathrm{N}_{3}\right)$ must increase; this is accomplished by converting one high-frequency 
quantum to two lower-frequency quantum, increasing the total number by one. Therefore, the condition that $\delta S>0$ forces energy to flow from $\mathrm{N}_{3}$ into $\mathrm{N}_{1}$ and $\mathrm{N}_{2}$.

One interesting fact that can be drawn from the above solution ([A.16]) is the saturation of energy transfer at large intensity ratios $\left(\mathrm{I}_{3} / \mathrm{I}_{2}>>\mathrm{e}^{\Gamma \mathrm{t}}\right)$. This arises from Eqn. [A.10], as the damped wave intensity $\mathrm{I}_{1}=\left|\mathrm{a}_{1}\right|^{2}$ is proportional to the product of the undamped intensities, $\mathrm{I}_{2} \mathrm{I}_{3}$, not the total amount of energy. Therefore changing an already-large intensity ratio while keeping $\mathrm{I}_{2}(0)+\mathrm{I}_{3}(0)$ constant will maintain a roughly constant ratio between the damped wave intensity $\mathrm{I}_{1}$ and the intensity of the weaker undamped wave. This, in turn, will "saturate" the energy transfer, as the same fraction of energy $\left(\mathrm{e}^{\Gamma \mathrm{t}}-1\right)$ will be transferred regardless of the actual ratio $\mathrm{I}_{3} / \mathrm{I}_{2}$.

\section{A.3 Steady State Solution}

An alternate solution to Eqns. [A.7] can be found via the "steady-state approximation", where the convective terms $(\mathrm{V} \delta \mathrm{a} / \delta \mathrm{x})$ are maintained and the time derivatives are ignored. This is allowable in certain situations, such as if the undamped waves $I_{2}$ and $I_{3}$ are laser beams that propagate through the interaction region much faster than the growth time of the damped wave $\mathrm{I}_{1}$. The mathematics is very similar to the above solution, and the 1-D solution is qualitatively similar because the new derivative only corresponds to a change in the rest frame of the problem. This approximation has recently been performed in two-dimensions for the particular case of two laser beams in resonance with an ion acoustic wave [33]. While the beam evolution was found to be highly 2-D and nonlinear, the energy transferred between the laser beams turned out to have a simple analytic solution. Because of the relevance to the experiment described in Chapter 3, the theoretical results will be summarized here.

After crossing with a higher-frequency wave in a plasma with resonant ion waves, the higher-frequency wave is found to lose energy by a fraction: 


$$
\frac{I_{3}(0)-I_{3}}{I_{3}(0)}=\frac{1}{g} \ln \left[e^{-r g}+e^{g}\left(1-e^{-r g}\right)\right]
$$

Here $\mathrm{r}=\mathrm{I}_{3}(0) / \mathrm{I}_{2}(0)$, and $\mathrm{g}$ is a normalized dimensionless parameter, proportional to both $\mathrm{I}_{1}$ and the gain length of the interaction. It is tedious to show the equivalence of [A.18] to the earlier solution [A.16] for small gain lengths, but in this regime there are no new results to be found in the low-gain limit of [A.18]. The important point is that the solution presented in section A.2 is still applicable for determining low-levels of resonant energy exchange between laser beams in situations relevant to the experiments in Chapter 3. Further discussion and comparison with these results can be found in that chapter.

\section{A.4 Saturation}

Finally, this analysis has all assumed that the damped wave is unsaturated. If some other process (besides damping) clamps $\mathrm{a}_{1}$ at some fixed amplitude, then Equation [A.10] is no longer valid, so (at saturation) $\mathrm{a}_{1}=\mathrm{A}_{1}=$ constant. The last two of [A.7] then become (in the steady-state approximation):

$$
\begin{aligned}
& \frac{\partial a_{2}}{\partial x}=\frac{\beta A_{1}}{V_{2}} a_{3} \\
& \frac{\partial a_{3}}{\partial x}=-\frac{\beta A_{1}}{V_{3}} a_{2}
\end{aligned}
$$

Taking another derivative and substituting:

$$
\begin{aligned}
& \frac{\partial^{2} a_{2}}{\partial x^{2}}=-\frac{\beta^{2} A_{1}^{2}}{V_{2} V_{3}} a_{2} \\
& \frac{\partial^{2} a_{3}}{\partial x^{2}}=-\frac{\beta^{2} A_{1}^{2}}{V_{2} V_{3}} a_{3}
\end{aligned}
$$

In the saturated limit, therefore, the solution is oscillatory. After a sufficient gainlength $\left\langle\mathrm{I}_{2}\right\rangle=\left\langle\mathrm{I}_{3}\right\rangle$, and statistical energy transfer can be expected if the original wave amplitudes are very different. For smaller gain lengths (over which pump depletion can 
be neglected), Equations [A.21-22] are no longer relevant. Instead, Equation [A.19] shows that $\mathrm{I}_{3}$ can be expected to transfer energy to $\mathrm{I}_{2}$ by an amount independent of the intensity of $\mathrm{I}_{2}$, until pump depletion lowers $\mathrm{I}_{3}$. Therefore calculating the "gain" from [A.19] and [A.20] in the absence of pump depletion:

$$
\text { Gain }=\frac{I_{2}(x)-I_{2}(0)}{I_{2}(0)}=\left(\frac{\beta A_{1} x}{V_{2}}\right)^{2} \frac{I_{3}}{I_{2}}
$$

This equation only holds for small gain lengths and assumes $I_{3}>>I_{2}$. Therefore the independence of intensity ratio (for high ratios) seen in [A.16] no longer holds in the saturated case; now higher intensity ratios continue to lead to higher gains of the lowerintensity wave. However, transition from the unsaturated to the saturated case (and the reverse) is a nonlinear problem that in general cannot be solved analytically. Therefore simulations are required in order to accurately model the saturation and subsequent relaxation of the damped plasma wave in three-wave resonance. 


\section{BIBLIOGRAPHY}

[1] A.S. Eddington, "The internal constitution of the stars", Report of the British Association for the Advancement of Science, (1920).

[2] R. Atkinson and F.G. Houtermans, Zeitschrift fur Physik, 54 (1929).

[3] E. Teller, Energy from Heaven and Earth, W.H. Freeman, San Francisco (1979).

[4] R. Herman, Fusion: the search for endless energy, Cambridge (1990).

[5] T.H. Maiman, Nature 187, 493 (1960).

[6] M. Tabak et al., Phys. Plasmas 1, 1626 (1994).

[7] C. Barty, Optics Letters 21, 219 (1996)

[8] R.A. Fisher and W.K. Bischell, IEEE J. Quant. Elec. 11, 46 (1975), D. Strickland and G. Mourou, Opt. Comm. 56, 219 (1985).

[9] V. Stefan, B.I. Coehn, and C. Joshi, Science 243, 494 (1989) and references therein.

[10] W.L. Kruer et al, Phys. Plasmas 3, 382 (1996).

[11] E.A. Williams et al., Phys. Plasmas 2, 129 (1995).

[12] B.B. Afeyan et al., Bull Am Phys Soc 40, 1822 (1995) and Phys. Plasmas, to be submitted; D.S. Montgomery et al., Phys. Plasmas 5, 1973 (1998).

[13] B.B. Afeyan, A.E. Chou and W.L. Kruer, ICF Quarterly Report, LLNL(1997) and submitted to Phys. Rev. E; B.B. Afeyan et al., Phys. Rev. Lett. 80, 2032 (1998).

[14] P. Kaw, G. Schmidt, and T. Wilcox, Phys. Fluids 16, 1522 (1972).

[15] A.J. Schmitt and B.B. Afeyan, Phys. Plasmas 5, 503 (1998).

[16] R.M. More et al, J. Physique C7 49, 43 (1988); E.G. Gamaly, Laser Part. Beams 12, 185 (1994).

[17] H.M. Milchberg and R.R. Freeman, J. Opt. Soc. Am. B 6, 1351 (1989); J.C. Kieffer et al, J. Quantum Electron. 25, 2640 (1989).

[18] G.J. Pert, Phys. Rev. E 51, 4778 (1995).

[19] V.L. Ginzburg, "The Propagation of Electromagnetic Waves in Plasmas" New York: Pergamon (1964).

[20] F. Brunel, Phys. Rev. Lett. 59, 52 (1987). 
[21] P. Gibbon and R. Forster, Plasma Phys. Control. Fusion 38, 769 (1996).

[22] W.L. Kruer and K. Estabrook, Phys. Fluids 28, 430 (1985)

[23] P. Gibbon and A.R. Bell, Phys. Rev. Lett. 68, 1535 (1992)

[24] S.C. Wilks, Phys. Fluids B 5, 2603 (1993).

[25] B. L. Stansfield, R. Nodwell and J. Meyer, Phys. Rev. Lett. 26, 1219 (1971); C. E. Clayton, C. Joshi, C. Darrow, and D. Umstadter, Phys. Rev. Lett. 54, 2343 (1985).

[26] C.E. Clayton et al, Phys. Rev. Lett. 70, 37 (1993).

[27] R.K. Kirkwood et al, submitted to Phys. Rev. Lett (1998).

[28] C.J. Pawley, H.E. Huey, and N.C. Luhmann, Jr., Phys. Rev. Lett. 49, 877 (1982);

C.W. Domier and N.C. Luhmann, Jr., Phys. Rev. Lett. 69, 3499 (1992).

[29] R.K. Kirkwood et al., Phys. Rev. Lett. 76, 2065 (1996).

[30] D.M. Villeneuve, H.A. Baldis, and J.E. Bernard, Phys. Rev. Lett. 59, 1585 (1987)

[31] A.K. Lal, K.A. Marsh, C.E. Clayton, C. Joshi, C.J. McKinstrie, J.S. Li, and T.W. Johnston, Phys. Rev. Lett. 78, 670 (1997).

[32] V.V. Eliseev, W. Rozmus, V.T. Tikhonchuk, and C.E. Capjack, Phys. Plasmas 3, 2215 (1996).

[33] C.J. McKinstrie, J.S. Li, R.E. Giacone, and H.X. Vu, Phys. Plasmas 3, 2686 (1996).

[34] I.M. Begg and R.A. Cairns, J. Phys. D 9, 2341 (1976).

[35] R.W. Short and E.A. Williams, Phys. Rev. Lett. 47, 337 (1981); C.J. Randall, J.R. Albritton, J.J. Thomson, Phys. Fluids 24, 1474 (1981); K. Baumgärtel and K. Sauer, Phys. Rev. A 26, 3031 (1982); R.W. Short and E.A. Williams, Phys. Fluids 26, 2342 (1983).

[36] R.K. Kirkwood et al., Phys. Plasmas 4, 1800 (1997); S.H. Glenzer et al., Phys. Plasmas., to be submitted.

[37] G. Zimmerman and W. Kruer, Comments Plasma Phys. Controlled Fusion 2, 85 (1975).

[38] K. Estabrook and D. Hinkle, private communication.

[39] W.L. Kruer, S.C. Wilks, B.B Afeyan, and R.K. Kirkwood, Phys. Plasmas 3, 382 (1996).

[40] C.J. McKinstrie, V.A. Smalyuk, R.E. Giacone and H.X. Vu, Phys. Rev. E 55, 2044 (1997). 
[41] H.A. Rose and S. Ghosal, Phys. Plasmas 5, 1461 (1998).

[42] S.N. Dixit, M.D. Feit, M.D. Perry, and H.T. Powell, Optics Lett. 21, 1715 (1996).

[43] S. Glenzer et al., Rev. Sci. Instrum. 68, 641 (1997).

[44] J.D. Moody, B.J. MacGowan, R.K. Kirkwood and D.S. Montgomery, Review of Sci. Instr. 68, 1725 (1997).

[45] B.I. Cohen, B.F. Lasinski, A.B. Langdon, and E.A. Williams, Phys. Plasmas 4, 965 (1997).

[46] B.I. Cohen et al., Phys. Plasmas 5, 3408 (1998).

[47] J.D. Hares et al., Phys. Rev. Lett. 42, 1216 (1979).

[48] N.A. Ebrahim, C. Joshi, and H.A. Baldis, Phys. Rev. A 25, 2440 (1982); B. LutherDavies, A. Perry, and K.A, Nugent. Phys. Rev. A 35, 4306 (1986).

[49] H. Chen et al., Phys. Rev. Lett. 70, 3431 (1993).

[50] A. Rousse et al., Phys. Rev. E 50, 2200 (1994).

[51] Z. Jiang et al., Phys. Plasmas 2, 1702 (1995).

[52] U. Teubner et al., Phys. Rev. E 54, 4167 (1996).

[53] F.N. Beg et al., Phys. Plasmas 4, 447 (1997).

[54] G. Malka and J.L. Miquel, Phys. Rev. Lett. 77, 75 (1996).

[55] N.F. Mott, H.S.W. Massey, "The Theory of Atomic Collisions", Clarendon, Oxford (1933).

[56] J.A. Halbleib and T.A. Mehlhorn, Nucl. Sci. Engr. 92, 338 (1986).

[57] B.C. Stuart et al., Optics Lett. 22242 (1997).

[58] Y. Zakharenkov et al., Rev Sci Instr 68, 847 (1997).

[59] C.E. Max et al., Phys. Rev. Lett. 33, 209 (1974); P. Sprangle et al., IEEE Trans. on Plasma Sci., PS-15, 145 (1987); W.B. Mori et al., Phys. Rev. Lett, 60, 1298 (1988).

[60] A. Puhkov and J. Meyer-ter-Vehn, Phys. Rev. Lett. 76, 3975 (1996).

[61] J. Koch et al., Lasers and Particle Beams 16, 225 (1998).

[62] S.C. Wilks et al., Phys. Rev. Lett. 69, 1383 (1992).

[63] M.G. Haines, Phys. Rev. Lett. 78, 254 (1997). 
[64] D.J. Bond, J.D. Hares, and J.D. Kilkenny, Phys. Rev. Lett., 45, 252 (1980).

[65] A.R. Bell et al., Plasma Phys. Control. Fusion 39, 653 (1997).

[66] J.R. Davies et al., Phys. Rev. E 56, 7193 (1997).

[67] A.P. Fews et al., Opt. Commun. 94, 259 (1992).

[68] M.D. Perry et al., submitted to Optics Letters (1998).

[69] Kent Estabrook, private communication.

[70] Denise Hinkel, private communication.

[71] Gail Glendinning, private communication.

[72] K. Nishikawa, in Advances in Plasma Physics, Vol. 6, New York (1976).

[73] J.M. Manley and H.E. Rowe, Proc. of the IRE, 44, 904 (1956).

[74] J.A. Armstrong, N.Bloembergen, J. Ducuing, and P.S.Pershan, Phys. Rev. 127, 1918 (1962). 

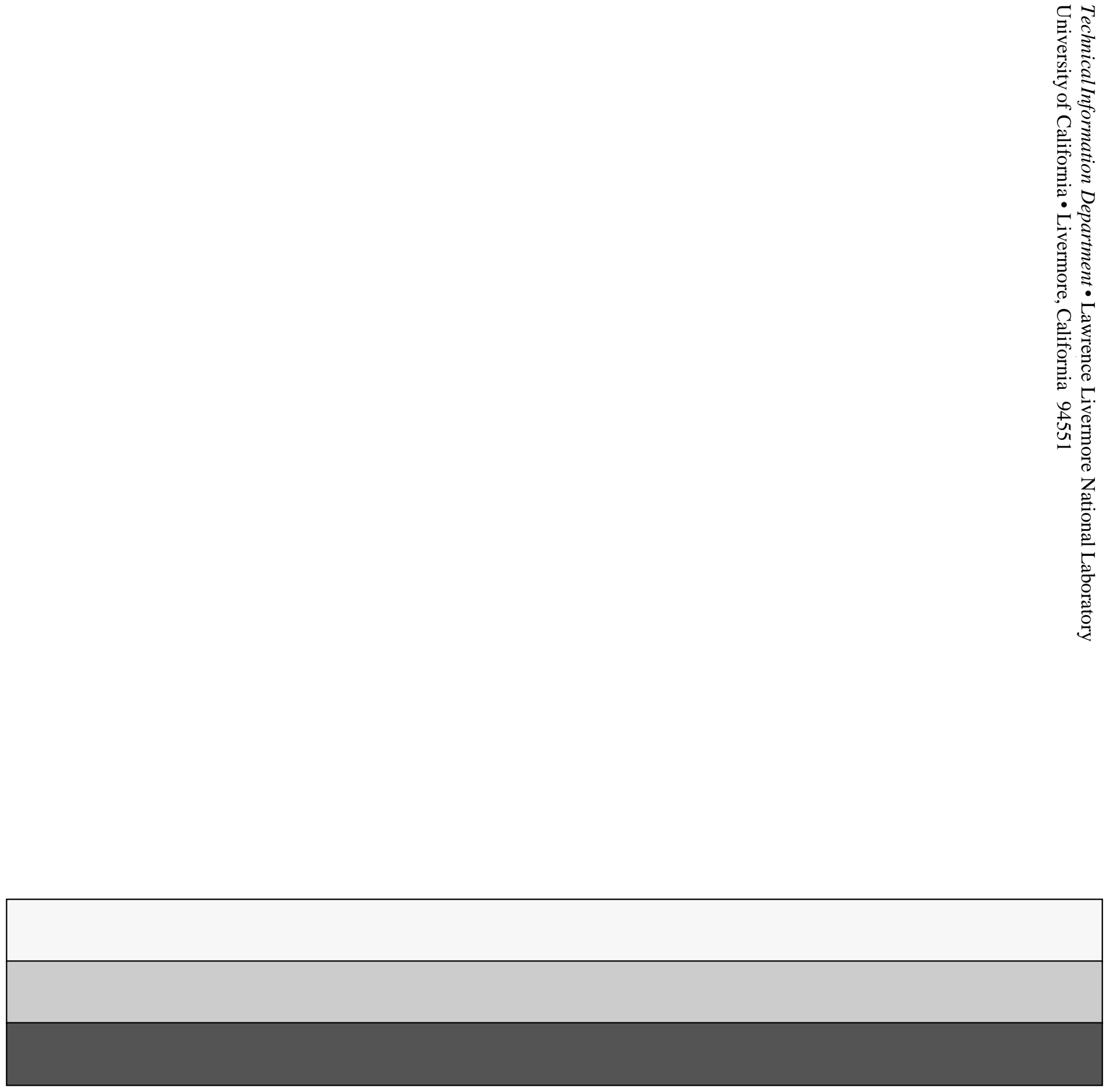